\title{
THE BARYON OSCILLATION SPECTROSCOPIC SURVEY OF SDSS-III
}

Kyle S. Dawson ${ }^{1}$, David J. Schlegel ${ }^{2}$, Christopher P. Ahn ${ }^{1}$, Scott F. Anderson ${ }^{3}$, Éric Aubourg ${ }^{4}$, Stephen Bailey $^{2}$, Robert H. Barkhouser ${ }^{5}$, Julian E. Bautista ${ }^{4}$, Alessandra Beifiori ${ }^{6}$, Andreas A. BerLind ${ }^{7}$, Vaishali Bhardwaj ${ }^{3}$,

Dmitry Bizyaev ${ }^{8}$, Cullen H. Blake ${ }^{9}$, Michael R. Blanton ${ }^{10}$, Michael Blomqvist ${ }^{11}$, Adam S. Bolton ${ }^{1}$, Arnaud Borde ${ }^{12}$, Jo Bovy ${ }^{13,75}$, W. N. Brandt ${ }^{14,15}$, Howard Brewington ${ }^{8}$, Jon BrinkmanN ${ }^{8}$, Peter J. Brown ${ }^{1,16}$, Joel R. Brownstein ${ }^{1}$, Kevin Bundy $^{17}$, N. G. Busca ${ }^{4}$, William Carithers ${ }^{2}$, Aurelio R. Carnero ${ }^{18,19}$, Michael A. Carr ${ }^{9}$, Yanmei Chen ${ }^{20,21}$, Johan Comparat ${ }^{22}$, Natalia Connolly ${ }^{23}$, Frances Cope $^{8}$, Rupert A. C. Croft ${ }^{24}$, Antonio J. Cuesta ${ }^{25}$, Luiz N. da Costa ${ }^{18,19}$, James R. A. Davenport ${ }^{3}$, Timothée Delubac ${ }^{12}$, Roland de Putter ${ }^{26,27}$, Saurav Dhital ${ }^{7,28}$, AnNe Ealet $^{29}$, Garrett L. EbelKe ${ }^{8}$, Daniel J. Eisenstein ${ }^{30}$, S. Escoffier ${ }^{29}$, XiaOhui Fan ${ }^{31}$, N. Filiz AK ${ }^{14,15,76}$, Hayley Finley ${ }^{32}$, Andreu Font-Ribera ${ }^{2,33}$, R. Génova-Santos ${ }^{34,35}$, James E. GunN ${ }^{9}$, Hong Guo ${ }^{36}$, Daryl HaGgard ${ }^{37}$, Patrick B. Hall ${ }^{38}$, Jean-Christophe Hamilton ${ }^{4}$, Ben Harris ${ }^{8}$, David W. Harris ${ }^{1}$, Shirley Ho $^{2,24}$, David W. Hogg ${ }^{10}$, Diana Holder ${ }^{8}$, Klaus Honscheid ${ }^{39}$, Joe HuehnerhofF ${ }^{8}$, Beatrice Jordan ${ }^{8}$, Wendell P. Jordan ${ }^{8}$,

Guinevere Kauffmann ${ }^{40}$, Eyal A. Kazin ${ }^{41}$, David Kirkby ${ }^{11}$, Mark A. Klaene ${ }^{8}$, Jean-Paul Kneib ${ }^{22}$, Jean-Marc Le GofF ${ }^{12}$, Khee-Gan LeE ${ }^{42}$, Daniel C. Long ${ }^{8}$, Craig P. Loomis ${ }^{9}$, Britt Lundgren ${ }^{25}$, Robert H. Lupton ${ }^{9}$, Marcio A. G. Maia ${ }^{18,19}$, Martin Makler $^{19,43}$, Elena Malanushenko ${ }^{8}$, Viktor Malanushenko ${ }^{8}$, Rachel Mandelbaum $^{9}, 24$, Marc Manera $^{44}$, Claudia Maraston ${ }^{44}$, Daniel Margala ${ }^{11}$, Karen L. Masters ${ }^{44}$, Cameron K. McBride ${ }^{30}$, Patrick McDonald ${ }^{2}$, Ian D. McGreer ${ }^{31}$, Richard G. McMahon ${ }^{45,46}$, Olga Mena ${ }^{27}$, Jordi Miralda-Escudé ${ }^{26,47}$, Antonio D. Montero-Dorta ${ }^{1,48}$, Francesco Montesano $^{6}$, Demitri Muna ${ }^{10}$, Adam D. Myers ${ }^{49}$, Tracy Naugle ${ }^{8}$, Robert C. Nichol ${ }^{44}$,

Pasquier Noterdaeme ${ }^{32}$, Sebastián E. Nuza ${ }^{50}$, Matthew D. Olmstead ${ }^{1}$, Audrey Oravetz ${ }^{8}$, Daniel J. Oravetz ${ }^{8}$, Russell Owen $^{3}$, Nikhil Padmanabhan ${ }^{25}$, Nathalie Palanque-Delabrouille ${ }^{12}$, Kaike Pan $^{8}$, John K. Parejko ${ }^{25}$, Isabelle PÂris ${ }^{32,51}$, Will J. Percival ${ }^{44}$, Ismael Pérez-Fournon ${ }^{34,35}$, Ignasi Pérez-RÀfols ${ }^{26}$, Patrick Petitjean ${ }^{32}$, Robert Pfaffenberger ${ }^{8}$, Janine Pforr 4,52 , Matthew M. Pieri ${ }^{44}$, Francisco Prada ${ }^{48,53,54}$, Adrian M. Price-Whelan ${ }^{55}$, M. Jordan Raddick ${ }^{56}$, Rafael Rebolo ${ }^{34,57}$, James Rich ${ }^{12}$, Gordon T. Richards ${ }^{58}$, Constance M. Rockosi ${ }^{59}$, Natalie A. RoE ${ }^{2}$, Ashley J. Ross ${ }^{44}$, Nicholas P. Ross ${ }^{2}$, Graziano Rossi ${ }^{60}$, J. A. Rubiño-Martin ${ }^{34,35}$, Lado Samushia ${ }^{44,61}$, Ariel G. SánCheZ ${ }^{6}$, Conor Sayres $^{3}$, Sarah J. Schmidt ${ }^{3}$, Donald P. Schneider ${ }^{14,15}$, C. G. Scóccola ${ }^{34,35}$, Hee-Jong Seo ${ }^{62}$, Alaina Shelden ${ }^{8}$,

Erin Sheldon ${ }^{63}$, Yue Shen ${ }^{30}$, Yiping Shu (舒轶平) ${ }^{1}$, Anže Slosar ${ }^{63}$, Stephen A. Smee ${ }^{5}$, Stephanie A. Snedden ${ }^{8}$,

Fritz Stauffer $^{8}$, Oliver Steele ${ }^{44}$, Michael A. Strauss ${ }^{9}$, Alina Streblyanska $^{34,35}$, Nao Suzuki ${ }^{2,64}$,

Molly E. C. Swanson ${ }^{30}$, Tomer TaL ${ }^{65}$, Masayuki Tanaka ${ }^{17}$, Daniel Thomas ${ }^{44}$, Jeremy L. Tinker ${ }^{10}$, Rita Tojeiro ${ }^{44}$, Christy A. Tremonti ${ }^{66}$, M. Vargas Magaña ${ }^{4}$, Licia Verde ${ }^{26,47}$, Matteo Viel ${ }^{67,68}$, David A. Wake ${ }^{65}$, Mike Watson ${ }^{69}$,

Benjamin A. Weaver ${ }^{10}$, David H. Weinberg ${ }^{70,71}$, Benjamin J. Weiner ${ }^{31}$, Andrew A. West ${ }^{28}$, Martin White ${ }^{2,64,72}$,

W. M. Wood-Vasey ${ }^{73}$, Christophe Yeche ${ }^{12}$, Idit Zehavi ${ }^{36}$, Gong-Bo Zhao ${ }^{44,74}$, and Zheng Zheng ${ }^{1}$

${ }^{1}$ Department of Physics and Astronomy, University of Utah, Salt Lake City, UT 84112, USA; kdawson@ astro.utah.edu

${ }^{2}$ Lawrence Berkeley National Laboratory, One Cyclotron Road, Berkeley, CA 94720, USA

${ }^{3}$ Department of Astronomy, University of Washington, Box 351580, Seattle, WA 98195, USA

${ }^{4}$ APC, University of Paris Diderot, CNRS/IN2P3, CEA/IRFU, Observatoire de Paris, Sorbonne Paris Cite, France

${ }^{5}$ Department of Physics and Astronomy, Johns Hopkins University, Baltimore, MD 21218, USA

${ }^{6}$ Max-Planck-Institut für Extraterrestrische Physik, Giessenbachstraße, D-85748 Garching, Germany

${ }^{7}$ Department of Physics and Astronomy, Vanderbilt University, VU Station 1807, Nashville, TN 37235, USA

${ }^{8}$ Apache Point Observatory, P.O. Box 59, Sunspot, NM 88349, USA

${ }^{9}$ Department of Astrophysical Sciences, Princeton University, Princeton, NJ 08544, USA

${ }^{10}$ Center for Cosmology and Particle Physics, Department of Physics, New York University, 4 Washington Place, New York, NY 10003, USA

${ }^{11}$ Department of Physics and Astronomy, University of California, Irvine, CA 92697, USA

${ }^{12}$ CEA, Centre de Saclay, Irfu/SPP, F-91191 Gif-sur-Yvette, France

${ }^{13}$ Institute for Advanced Study, Einstein Drive, Princeton, NJ 08540, USA

${ }^{14}$ Department of Astronomy and Astrophysics, 525 Davey Laboratory, The Pennsylvania State University, University Park, PA 16802, USA

${ }^{15}$ Institute for Gravitation and the Cosmos, The Pennsylvania State University, University Park, PA 16802, USA

${ }^{16}$ George P. and Cynthia Woods Mitchell Institute for Fundamental Physics \& Astronomy, Department of Physics and Astronomy, Texas A. \& M. University, 4242 TAMU, College Station, TX 77843, USA

${ }^{17}$ Kavli Institute for the Physics and Mathematics of the Universe, Todai Institutes for Advanced Study The University of Tokyo, Kashiwa, 277-8583, Japan (Kavli IPMU, WPI)

${ }^{18}$ Observatório Nacional, Rua Gal. José Cristino 77, Rio de Janeiro, RJ-20921-400, Brazil

${ }^{19}$ Laboratório Interinstitucional de e-Astronomia, LIneA, Rua Gal. José Cristino 77, Rio de Janeiro, RJ-20921-400, Brazil

${ }^{20}$ Department of Astronomy, Nanjing University, Nanjing 210093, China

${ }^{21}$ Key Laboratory of Modern Astronomy and Astrophysics (Nanjing University), Ministry of Education, Nanjing 210093, China

${ }^{22}$ Aix Marseille Université, CNRS, LAM (Laboratoire d'Astrophysique de Marseille), UMR 7326, F-13388 Marseille, France

${ }^{23}$ Department of Physics, Hamilton College, Clinton, NY 13323, USA

${ }^{24}$ Bruce and Astrid McWilliams Center for Cosmology, Department of Physics, Carnegie Mellon University, 5000 Forbes Ave, Pittsburgh, PA 15213, USA

${ }^{25}$ Yale Center for Astronomy and Astrophysics, Yale University, New Haven, CT 06520, USA

${ }^{26}$ Institut de Ciències del Cosmos, Universitat de Barcelona/IEEC, E-08028 Barcelona, Spain

${ }^{27}$ Instituto de Fisica Corpuscular, University of Valencia-CSIC, E-46971 Valencia, Spain

${ }^{28}$ Department of Astronomy, Boston University, 725 Commonwealth Avenue, Boston, MA 02215, USA

${ }^{29}$ Centre de Physique des Particules de Marseille, Aix-Marseille Université, CNRS/IN2P3, F-13288 Marseille, France

${ }^{30}$ Harvard-Smithsonian Center for Astrophysics, Harvard University, 60 Garden St., Cambridge, MA 02138, USA 


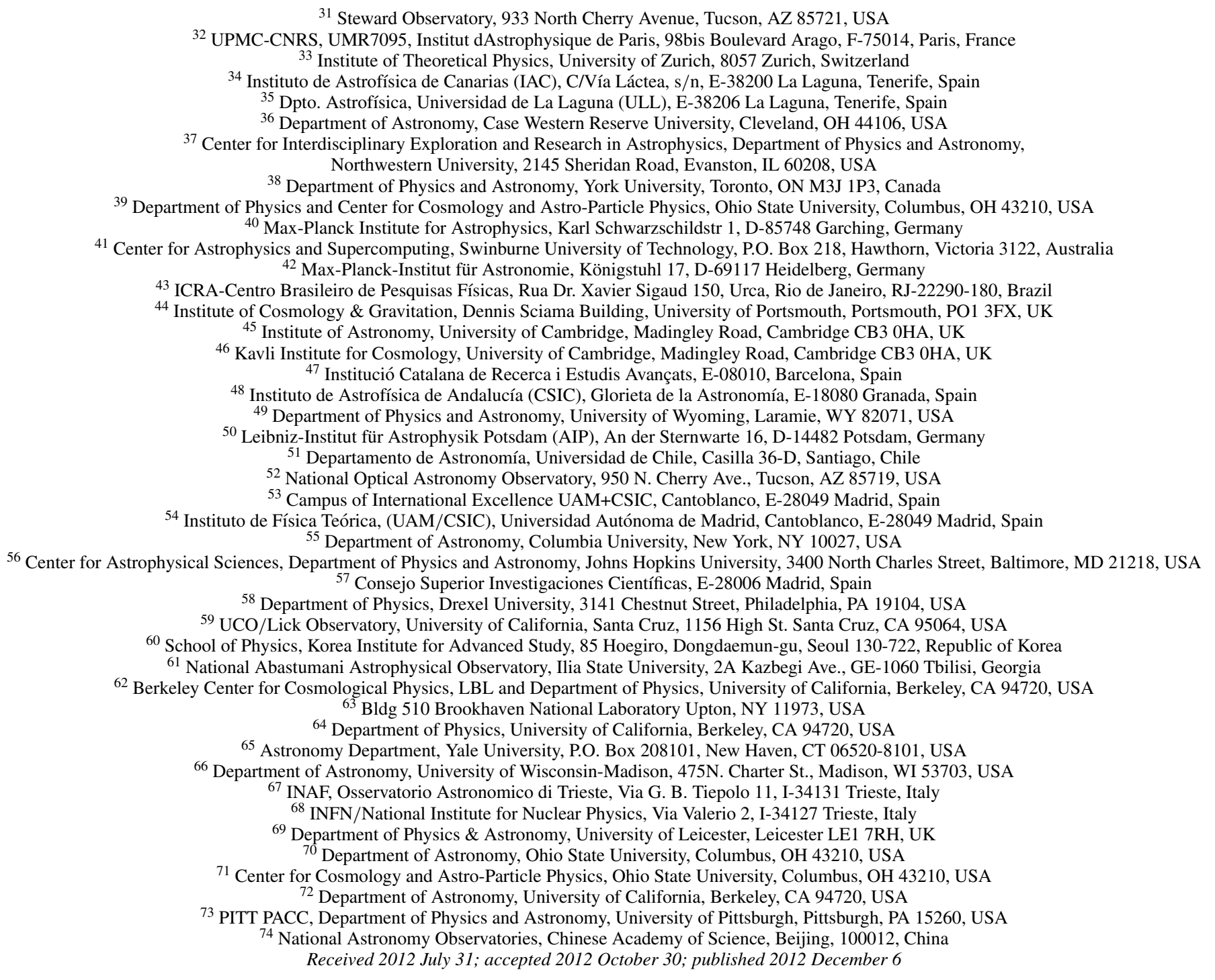

\begin{abstract}
The Baryon Oscillation Spectroscopic Survey (BOSS) is designed to measure the scale of baryon acoustic oscillations (BAO) in the clustering of matter over a larger volume than the combined efforts of all previous spectroscopic surveys of large-scale structure. BOSS uses 1.5 million luminous galaxies as faint as $i=19.9$ over $10,000 \mathrm{deg}^{2}$ to measure BAO to redshifts $z<0.7$. Observations of neutral hydrogen in the Ly $\alpha$ forest in more than 150,000 quasar spectra $(g<22)$ will constrain BAO over the redshift range $2.15<z<3.5$. Early results from BOSS include the first detection of the large-scale three-dimensional clustering of the Ly $\alpha$ forest and a strong detection from the Data Release 9 data set of the BAO in the clustering of massive galaxies at an effective redshift $z=0.57$. We project that BOSS will yield measurements of the angular diameter distance $d_{A}$ to an accuracy of $1.0 \%$ at redshifts $z=0.3$ and $z=0.57$ and measurements of $H(z)$ to $1.8 \%$ and $1.7 \%$ at the same redshifts. Forecasts for $\operatorname{Ly} \alpha$ forest constraints predict a measurement of an overall dilation factor that scales the highly degenerate $D_{A}(z)$ and $H^{-1}(z)$ parameters to an accuracy of $1.9 \%$ at $z \sim 2.5$ when the survey is complete. Here, we provide an overview of the selection of spectroscopic targets, planning of observations, and analysis of data and data quality of BOSS.
\end{abstract}

Key words: cosmology: observations - surveys

Online-only material: color figures

\title{
1. INTRODUCTION
}

\footnotetext{
75 Hubble fellow.

${ }^{76}$ Faculty of Sciences, Department of Astronomy and Space Sciences, Erciyes University, 38039 Kayseri, Turkey.
}

The large-scale structure of the universe, as traced by galaxies in redshift surveys and by intergalactic hydrogen absorption toward distant quasars, has played a central role in establishing 
the modern cosmological model based on inflation, cold dark matter, and dark energy. Key steps in this development have included the Center for Astrophysics redshift surveys (Huchra et al. 1983; Falco et al. 1999), the Las Campanas Redshift Survey (Shectman et al. 1996), and the Two Degree Field Galaxy Redshift Survey (2dFGRS; Colless et al. 2001). The largest and most powerful redshift surveys to date have been those from the Sloan Digital Sky Survey (SDSS; York et al. 2000), which measured redshifts of nearly one million galaxies in spectroscopic observations between 2000 and 2008 (the phases known as SDSS-I and II, described in the seventh data release, DR7, by Abazajian et al. 2009). The SDSS also obtained the most precise constraints to date on structure at $2<z<4$ using Ly $\alpha$ forest absorption toward $~ 3000$ high-redshift quasars (McDonald et al. 2005, 2006; Seljak et al. 2006), building on earlier studies that analyzed much smaller samples of higher resolution spectra (Croft et al. 1999, 2002; McDonald et al. 2000; Kim et al. 2004).

This paper describes the Baryon Oscillation Spectroscopic Survey (BOSS), the largest of the four surveys that comprise SDSS-III (Eisenstein et al. 2011). Shorter summaries of BOSS have appeared previously in an Astro2010 white paper (Schlegel et al. 2009), in the SDSS-III overview paper (Eisenstein et al. 2011), and in papers presenting some of the survey's early cosmological results (e.g., White et al. 2011; Slosar et al. 2011; Anderson et al. 2012).

Large-scale structure offers a novel tool to make precise measurements of cosmic distances via baryon acoustic oscillations (BAO), a feature imprinted on the clustering of matter by acoustic waves that propagate in the pre-recombination universe. A long-standing prediction of cosmological models (Sakharov 1966; Peebles \& Yu 1970; Sunyaev \& Zel'dovich 1970), BAO rose to prominence in recent years as a tool to measure the expansion history of the universe (Eisenstein et al. 1998; Blake \& Glazebrook 2003; Seo \& Eisenstein 2003). The first clear detections of BAO came in 2005 from analyses of the SDSS (Eisenstein et al. 2005) and the 2dFGRS (Cole et al. 2005) galaxy samples, and even these first discoveries set few-percent constraints on the cosmic distance scale. With sufficient data, the BAO "standard ruler" can be used to separately measure the angular diameter distance $D_{A}(z)$ from transverse clustering and the Hubble expansion rate $H(z)$ from line-of-sight clustering. $D_{A}(z)$ and $H(z)$ have not yet been measured separately at any redshift. A particular attraction of the BAO method is its low susceptibility to systematic errors, a feature highlighted in the report of the Dark Energy Task Force (DETF; Albrecht et al. 2006). The BAO method is reviewed in detail by Weinberg et al. (2012, see their Section 4), including discussions of the underlying theory, the effects of nonlinear evolution and galaxy bias, survey design and statistical errors, control of systematics, recent observational results, and complementarity with other probes of cosmic acceleration.

A number of recent cosmological surveys were designed with BAO measurement as a major goal. The recently completed WiggleZ survey (Drinkwater et al. 2010) observed 200,000 emission-line galaxies over $800 \mathrm{deg}^{2}$, obtaining the first BAO measurements at $0.5<z<1.0$, with an aggregate distance precision of $3.8 \%$ at a central redshift $z=0.6$ (Blake et al. 2011b). The Six-Degree Field Galaxy Survey (Jones et al. 2009) was designed to map structure in the local universe; it produced a $4.5 \%$ BAO distance measurement at redshift $z \sim 0.1$ (Beutler et al. 2011). The primary SDSS BAO results came from its redshift survey of luminous red galaxies (LRGs; Eisenstein et al.
2001), with color selection used to separate high luminosity targets from nearby galaxies at similar apparent magnitudes. This survey, reaching a limiting apparent magnitude $r=19.5$, provided a sparse sample of 106,000 strongly clustered galaxies over a large volume, well suited to measuring structure on BAO scales. The LRG survey achieved a roughly constant comoving space density of $10^{-4} h^{3} \mathrm{Mpc}^{-3}$ from $z=0.16$ to $z=0.40$, with a declining density out to $z \sim 0.45$. Percival et al. (2010) derived the power spectrum from the LRG sample, the DR7 galaxy sample, and the $2 \mathrm{dFGRS}$ sample to obtain a $2.7 \%$ measurement of the BAO scale at $z=0.275$ using a total of 893,319 galaxies over $9100 \mathrm{deg}^{2}$. Kazin et al. (2010) used the full LRG sample from DR7 to measure the galaxy correlation function and obtain a $3.5 \%$ measurement of the BAO distance scale at $z=0.35$.

The BAO measurements described above suffer from a degradation in the detection significance due to large-scale flows and nonlinear evolution in the density field. As matter diffuses due to nonlinear evolution and galaxy peculiar velocities, the BAO peak becomes broader and more difficult to constrain, particularly at low redshift. These effects can partly be reversed by "reconstruction," a technique by which the observed galaxy field is used to estimate the large-scale gravitational potential (Eisenstein et al. 2007). Using the Zel'dovich approximation (Zel'dovich 1970), the diffusion of galaxies can then be measured and the density fluctuations can be shifted back to their Lagrangian positions, thereby restoring the acoustic peak in both real and redshift space. Redshift-space distortions (Kaiser 1987) are removed in the same manner. The approach was refined, tested on simulated data, and first applied to survey data using the full SDSS LRG sample (Padmanabhan et al. 2012). The scale of the acoustic peak was measured from the reconstructed data to an accuracy of $1.9 \%$ (Xu et al. 2012), an improvement of almost a factor of two over the $3.5 \%$ measurement before reconstruction. Combining this distance measurement with the WMAP7 cosmic microwave background (CMB) anisotropies (Komatsu et al. 2011) leads to an estimate of $H_{0}=69.8 \pm 1.2 \mathrm{~km} \mathrm{~s}^{-1} \mathrm{Mpc}^{-1}$ and $\Omega_{M}=0.280 \pm 0.014$ for a flat universe with a cosmological constant $(w=-1)$. The cosmological constraints are additionally improved when the reconstructed SDSS data are combined with Type Ia supernovae (SNe Ia) from the SN Legacy Survey (Conley et al. 2011), measurements of $H_{0}$ from the Hubble Space Telescope (HST; Riess et al. 2011), and BAO constraints from the 6dF Galaxy Survey (Beutler et al. 2011) and the WiggleZ Dark Energy Survey (Blake et al. 2011a). Mehta et al. (2012) use these measurements to constrain $w=-1.03 \pm 0.08$ for a cosmological model in which the equation of state for dark energy is allowed to vary.

As the name suggests, BOSS is a survey designed to measure the universe using BAO. BOSS uses a rebuilt spectrograph from the original SDSS survey with smaller fibers, new improved detectors, higher throughput, and a wider wavelength range, enabling a spectroscopic survey to higher redshift and roughly 1 mag deeper than SDSS. We hereafter refer to the upgraded instruments as the BOSS spectrographs. The BOSS spectrographs and their SDSS predecessors are mounted to the telescope and are described in detail by Smee et al. (2012). In brief, there are two double spectrographs, each covering the wavelength range $361-1014 \mathrm{~nm}$ with resolving power $\lambda / \Delta \lambda$ ranging from 1300 at the blue end to 2600 at the red end. Both spectrographs have a red channel with a $4 \mathrm{k} \times 4 \mathrm{k}, 15 \mu \mathrm{m}$ pixel CCD (Holland et al. 2006) from Lawrence Berkeley National Laboratory (LBNL). We denote these channels as R1 and R2 for the first and second spectrographs, respectively. Similarly, both spectrographs have 
a blue channel with a $4 \mathrm{k} \times 4 \mathrm{k}, 15 \mu \mathrm{m}$ pixel CCD from $\mathrm{e} 2 \mathrm{v}$ denoted B1 and B2. The instrument is fed by 1000 optical fibers (500 per spectrograph), each subtending $2^{\prime \prime}$ on the sky. (The original spectrographs used $6403^{\prime \prime}$ fibers.) When the survey is complete, fibers will have been plugged into more than 2000 unique spectroscopic plates that each cover a circular field of view with $3^{\circ}$ diameter. Aluminum cast cartridges support the optical fibers, spectrograph slithead, and spectroscopic plug plate.

BOSS consists primarily of two interleaved spectroscopic surveys observed simultaneously: a redshift survey of 1.5 million luminous galaxies extending to $z=0.7$ and a survey of the Ly $\alpha$ forest toward 150,000 quasars in the redshift range $2.15 \leqslant z \leqslant 3.5$. BOSS uses the same wide field, dedicated telescope as was employed by SDSS-I and II, the $2.5 \mathrm{~m}$ aperture Sloan Foundation Telescope at Apache Point Observatory in New Mexico (Gunn et al. 2006). Those surveys imaged more than $10000 \mathrm{deg}^{2}$ of high latitude sky in the ugriz bands (Fukugita et al. 1996), using a mosaic CCD camera with a field of view spanning 2.5 (Gunn et al. 1998). As discussed in Section 2.1, SDSS-III completed roughly $2500 \mathrm{deg}^{2}$ of new imaging to enlarge the footprint available to BOSS. All of the imaging, including that from SDSS-I and II, is available in SDSS Data Release 8 (DR8; Aihara et al. 2011a), the first data release from SDSS-III. The forthcoming Data Release 9 (Ahn et al. 2012) will present the first public release of BOSS spectroscopic data, containing observations completed prior to 2011 August.

The defining goal of the BOSS galaxy survey is to produce BAO measurements limited mainly by sample variance (as opposed to galaxy shot noise) over the volume available to the Sloan $2.5 \mathrm{~m}$ telescope, a volume defined by the area of accessible high latitude sky and by the maximum practical redshift depth. The BOSS survey observes primarily at $\delta>-3.75$ in the SDSS high-Galactic latitude footprint. The spectroscopic footprint is approximately $10,000 \mathrm{deg}^{2}$; larger areas begin to stray into regions of high Galactic extinction. Given the configuration of the BOSS experiment, we can exceed the density of objects in the LRG sample and observe a comoving space density $\bar{n}=2-3 \times 10^{-4} h^{3} \mathrm{Mpc}^{-3}$ with strongly clustered galaxies (bias factor $b \sim 2$ ). In $1 \mathrm{hr}$ exposures under good conditions, the BOSS spectrographs can measure redshifts of these luminous galaxies with high completeness to $i=19.9$, which for the desired space density puts the outer redshift limit at $z=0.7$. BOSS is designed to observe 1.5 million galaxies to these limits, including 150,000 galaxies that satisfy the BOSS selection criteria but were previously observed during SDSS-I and II. For BAO and other large-scale power spectrum measurements, the comoving volume of the completed BOSS galaxy survey, weighted by the redshift-dependent number density and galaxy power spectrum, will be 6.3 times that of the SDSS-I and II LRG samples. The higher density of BOSS galaxies relative to SDSS-I and II LRGs also improves the performance of the reconstruction methods discussed above that correct for nonlinear degradation of the BAO signal.

The BOSS quasar survey is pioneering a novel method of measuring BAO at high redshift $(2.15<z<3.5)$ using Ly $\alpha$ forest absorption toward a dense grid of background quasars. The redshifted Ly $\alpha$ line becomes detectable in the BOSS spectral range just beyond $z=2$ and becomes highly opaque at $z>4$, motivating the targeted redshift range. Even at moderate resolution, transmitted flux in the forest provides a measure of hydrogen along the line of sight that can be used to infer clustering of the underlying dark matter distribution (Croft et al. 1998, 1999; McDonald 2003). Prior to BOSS, cosmological measurements of the forest treated each quasar sight line in isolation, since samples were too small and too sparse to measure correlations across sight lines except for a few cases of close pairs or other tight configurations. McDonald (2003) and White (2003) suggested that three-dimensional Ly $\alpha$ forest correlations in a large quasar survey could be used to measure BAO. McDonald \& Eisenstein (2007) developed this idea in detail, constructing a Fisher matrix formalism that helped guide the design of the BOSS quasar survey. Slosar et al. (2011) used the first year of BOSS data to obtain the first threedimensional measurement of large-scale structure in the forest, detecting transverse flux correlations to comoving separations of at least $60 \mathrm{~h}^{-1} \mathrm{Mpc}$. The goal of the BOSS quasar survey is to obtain spectra of at least 150,000 Ly $\alpha$ quasars selected from about 400,000 targets. Extrapolations based on data taken to date suggest that BOSS will ultimately observe about 175,000 Ly $\alpha$ quasars over 10,000 $\mathrm{deg}^{2}$. Ultraviolet, near-infrared, and multiple-epoch optical imaging data increased the selection efficiency beyond the minimum goal.

This paper is one of a series of technical papers describing the BOSS survey. The BOSS imaging survey data are described in Aihara et al. (2011a), while BOSS is described in the context of SDSS-III in Eisenstein et al. (2011). The selection of galaxy and quasar targets are described in N. Padmanabhan et al. (2013, in preparation) and Ross et al. (2012b), respectively, the spectrograph design and performance are discussed in Smee et al. (2012), and the spectral data reductions are described in D. Schlegel et al. (2013, in preparation) and Bolton et al. (2012b). Here, we describe the details of the BOSS survey itself, with an emphasis on survey strategy and operations. In Section 2, we present the BOSS footprint and the selection of the galaxies and quasars that will be used to measure the BAO feature. In Section 3, we review the process by which we design the spectroscopic plates to observe those targets. In Section 4, we describe the procedures for observation, the real-time data quality assessment, and the data processing pipeline. Much of the information found in Sections 3 and 4 was never described for SDSS-I or -II and remains unchanged from those earlier surveys. We discuss the redshift efficiency and the strategy to complete observations of the full $10,000 \mathrm{deg}^{2}$ in Section 5 and provide examples of the spectral data quality in Section 6. Finally, we compare the BOSS data to SDSS spectra, highlight some recent BOSS science results, and provide forecasts for cosmological constraints in Section 7.

\section{SPECTROSCOPIC TARGETS}

The SDSS-I and II imaging programs provide two large, contiguous regions of sky that lie away from the Galactic plane. Additional imaging in the Falls of 2008 and 2009 extended this footprint and increased the sky volume observable with BOSS. BOSS is designed to survey the full SDSS imaging footprint with dense spectroscopic coverage over five years. The first goal of BOSS is a redshift survey of $\sim 1.5$ million luminous galaxies at $0.15<z<0.7$, at a surface density of $150 \mathrm{deg}^{-2}$. This sample is divided into two subsamples. The first is a low-redshift sample at $0.15<z<0.43$, with a median redshift of $z=0.3$ and a surface density of $30 \mathrm{deg}^{-2}$. This sample is a simple extension of the SDSS-I and II LRG samples (Eisenstein et al. 2001) to lower luminosities, where the brightest $\sim 10 \mathrm{deg}^{-2}$ have already been observed by SDSS. A higher redshift sample expands the 2dF-SDSS LRG and QSO Survey (2SLAQ; Cannon et al. 2006) galaxy selections and covers $0.43<z<0.7$, with a surface 
density of $120 \mathrm{deg}^{-2}$ and a median redshift of $z=0.57$. The second goal of BOSS is to survey at least 150,000 quasar spectra at $2.15<z<3.5$ to probe the intergalactic medium along each sight line through $\operatorname{Ly} \alpha$ absorption. Approximately $5 \%$ of the BOSS fibers are allocated to obtain spectra of targets that would otherwise not be included in the BOSS target selection. Dubbed ancillary science targets, these spectra provide the data for smaller research programs proposed by members of the collaboration. These programs are described in Appendix B.

\subsection{Imaging Data}

In its first five years of operation (2000-2005), SDSS obtained five-band imaging over $7600 \mathrm{deg}^{2}$ of the high Galactic latitude sky in the northern Galactic hemisphere and $600 \mathrm{deg}^{2}$ along four disjoint stripes in the southern hemisphere. During the falls of 2008 and 2009, the same camera (Gunn et al. 1998) was used to grow the southern imaging to a contiguous $3100 \mathrm{deg}^{2}$ footprint. As with the original SDSS imaging survey, we obtained CCD imaging in scans with an effective exposure time of $55 \mathrm{~s}$ in each of five filters (ugriz; Fukugita et al. 1996). Imaging was performed when the moon was below the horizon, under photometric conditions, and seeing was better than $2^{\prime \prime}$ in the $r$ filter. The prioritization was to image north of the celestial equator in September and October, and south of the equator in November and December. This prioritization makes the imaging depth more uniform by roughly canceling two effects: the southern declinations are looking through more atmosphere, but we did so in the winter months when the atmosphere is typically more transparent. The seeing requirements were somewhat relaxed from SDSS by accepting conditions to $2^{\prime \prime}$, although the minimum scan time was increased from 0.5 to $1 \mathrm{hr}$ to ensure sufficient astrometric and photometric calibration stars. The typical $50 \%$ completeness limit for detection of point sources is $r=22.5$.

The final SDSS imaging data set, including the new BOSS imaging, was released as part of DR8. These data were reduced with a uniform version of the photometric pipeline (Lupton et al. 2001; Stoughton et al. 2002) with improved sky subtraction described in the DR8 release (Aihara et al. 2011a). The photometric calibration (Smith et al. 2002; Ivezić et al. 2004; Tucker et al. 2006) has been improved with a global re-calibration (Padmanabhan et al. 2008). The astrometric (Pier et al. 2003) calibration was tied to the UCAC2 system for declinations below approximately $41^{\circ}$ and the USNO-B system at higher declinations. This astrometry is less accurate than the earlier DR7 release or the later re-calibration of the entire sky in the DR9 release. The BOSS coordinates for all target classes correspond to the DR8 astrometric system, which is internally consistent and only introduces small relative offsets on the $3^{\circ}$ scale of a plate. These coordinates are offset 240 mas to the north and 50 mas to the west relative to DR9 coordinates at higher declinations, with additional sources of error introducing scatter of $\sim 50$ mas (Ahn et al. 2012).

These imaging data define the baseline goal for the BOSS spectroscopic survey. We used a trimmed subset called the "BOSS footprint," which is two contiguous regions of low extinction centered in the north Galactic cap (NGC) and south Galactic cap (SGC). To avoid regions of high extinction, the footprint lies above $\pm 25^{\circ}$ Galactic latitude for all regions except around a right ascension of $120^{\circ}\left(b>15^{\circ}\right)$ and right ascension of $330^{\circ}\left(b>20^{\circ}\right)$. Several 0.2 wide strips were also excised from this overall footprint for the quasar targets only to reject imaging data when $u$-band amplifiers were not functioning, as

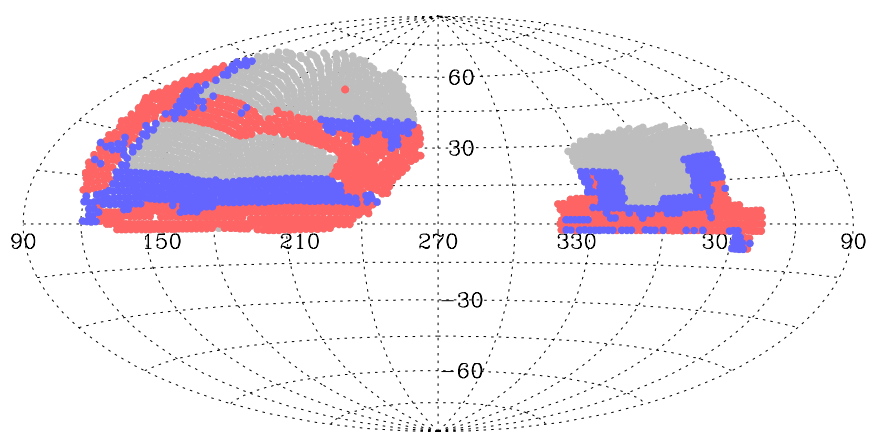

Figure 1. Location of pointing centers for the 2208 spectroscopic plates in the BOSS survey footprint in an Aitoff projection in J2000 equatorial coordinates. Gray circles represent the location of plates that remained to be drilled after the summer of 2011. Blue circles represent plates that were drilled and were ready to observe in the third year of the survey. Red circles represent the plates that were completed in either the first or second year of the survey, whose observations are released in DR9.

(A color version of this figure is available in the online journal.)

explained in DR2 (Abazajian et al. 2004). The BOSS footprint is presented in Figure 1.

In total, galaxy spectroscopic targets were chosen from $3172 \mathrm{deg}^{2}$ in the SGC and $7606 \mathrm{deg}^{2}$ in the NGC. A total of $7578 \mathrm{deg}^{2}$ in the NGC were used for the quasar target selection due to the loss of $u$-band imaging in certain regions. Because this footprint is too large for the BOSS survey, it was further trimmed by removing most of the area south of the celestial equator, reducing the SGC footprint to $2663 \mathrm{deg}^{2}$. The coverage of the SGC is presented in Figure 1 as the region with $\alpha>300^{\circ}$ or $\alpha<60^{\circ}$. The coverage of the NGC is included in the region with $60^{\circ}<\alpha<300^{\circ}$. The spur in Figure 1 located at approximately $30^{\circ}<\alpha<40^{\circ}$ and $\delta<-2^{\circ}$ was chosen to overlap with the W1 region of the Canada-France-Hawaii Telescope Legacy Survey (CFHTLS; Goranova et al. 2009). Using ugriz photometry from these $10,269 \mathrm{deg}^{2}$, we choose galaxy, quasar, and ancillary science targets for spectroscopic follow-up. A summary of the area covered in imaging and spectroscopy is provided in Table 1.

\subsection{Imaging Pre-selection}

We briefly describe those photometric parameters measured for each object in the imaging data that are relevant to target selection. More detailed descriptions are available in the online SDSS documentation and the data release papers, especially EDR (Stoughton et al. 2002) and DR2 (Abazajian et al. 2004).

1. PSF magnitudes are designed for point sources and are a fit of the point-spread function (PSF) model evaluated at the location of each object. We refer to these magnitudes measured in any filter " $X$ " as $X_{\mathrm{PSF}}$.

2. Fiber2 magnitudes are designed to simulate the flux captured by the BOSS fibers for either point sources or extended sources. The images are convolved with Gaussians to simulate $2^{\prime \prime}$ seeing, and the flux is measured within a $2^{\prime \prime}$ circular aperture representing the size of the BOSS fibers. Unlike the other magnitudes that are measured on individual objects deblended from their neighbors, the fiber magnitudes include flux from those neighbors. A caveat of these magnitudes is that for the small amount of SDSS imaging data taken in seeing worse than $2^{\prime \prime}$ seeing, the fiber magnitudes are measured without convolution or deconvolution of those images. In those cases, the fiber magnitudes are fainter than they should be. Throughout the text, we refer to these magnitudes measured in any filter " $X$ " as $X_{\mathrm{fib} 2}$. These 
Table 1

Survey Parameters

\begin{tabular}{lcccr}
\hline \hline Region & $\begin{array}{c}\text { Contiguous Imaging } \\
\text { Area }\left(\mathrm{deg}^{2}\right)\end{array}$ & $\begin{array}{c}\text { Area for Galaxy } \\
\text { Targets }\left(\mathrm{deg}^{2}\right)\end{array}$ & $\begin{array}{r}\text { Area for Quasar } \\
\text { Targets }\left(\mathrm{deg}^{2}\right)\end{array}$ & $\begin{array}{r}\text { Area after } \\
\text { Tiling }(\mathrm{deg}\end{array}$ \\
\hline SGC & 3172 & 2663 & 2663 & $\begin{array}{r}\text { Number } \\
\text { of Plates }^{2}\end{array}$ \\
NGC & 7606 & 7606 & 7578 & 633 \\
\hline
\end{tabular}

magnitudes are analogous to the $3^{\prime \prime}$ fiber magnitudes from SDSS, which simulated the flux captured by the original SDSS 3 " fibers.

3. Model magnitudes are designed to measure galaxy colors that are unbiased relative to the signal-to-noise ratio $(\mathrm{S} / \mathrm{N})$ of the image or unmodeled substructure in the galaxies. A library of de Vaucouleurs (de Vaucouleurs 1948) and exponential (Freeman 1970) profiles with varying radii, ellipticities, and position angles is fit to each object's image. The best-fit profile from the $r$ band, convolved with the PSF in each band, is used as a matched aperture for the model magnitudes.

4. Cmodel magnitudes (composite model) are designed to produce a good estimate of the "total" flux of galaxies. These magnitudes are based upon the best-fitting positive linear combination of exponential and de Vaucouleurs profiles, convolved with the PSF. These are measured independently in each band.

5. $r_{d e V, i}$ is the best-fit de Vaucouleurs effective radius in the $i$ band (in units of arcseconds).

Every object in the SDSS imaging catalogs has a series of flags indicating the nature and quality of the data, allowing one to define a single unique measurement of objects in regions observed more than once, and to identify any problems with processing and measuring the properties of each object. We used these flags to remove questionable objects from consideration for spectroscopy.

Repeat observations and overlapping scans resulted in multiple observations for some objects; only unique observations identified by the SURVEY_PRIMARY bit of the RESOLVE_STATUS flag assigned to each object were used for the main targeting algorithms, as described in Aihara et al. (2011a). Multiple imaging observations were used for some special programs, such as quasars targeted based on their variability.

For the galaxy, quasar, and standard star targets selected for the primary BAO program (denoted "main targets" in which follows), we rejected objects for which flags set during photometric pipeline processing ${ }^{77}$ indicated that their photometry was unreliable. All objects with the SATUR flag set (which indicates that the object includes saturated pixels) are dropped from consideration for galaxies, quasars, and standard stars.

The quasar target selection algorithm is most sensitive to objects with unreliable photometry (stars are far more numerous, leading to a very large contamination rate), and thus it does the most careful checking. The flags used are described in detail in Bovy et al. (2011a) and Appendix A of Ross et al. (2012b; see also Richards et al. 2006), and include flags designed to indicate problems due to deblending, interpolation over cosmic rays and bad pixels and columns, and proper motion between exposures in different filters (due to asteroids).

For galaxy target selection, we also use a specialized set of flag cuts to ensure good target selection. The detailed galaxy

\footnotetext{
77 http://www.sdss3.org/dr8/algorithms/flags_detail.php
}

flags for galaxies are given in N. Padmanabhan et al. (2013, in preparation).

Objects flagged in the following ways were not considered for followup as standard stars. These flag cuts are in addition to SURVEY_PRIMARY and SATUR listed above.

1. Objects observed in non-photometric conditions (as indicated by the CALIB_STATUS flag) in any band.

2. Objects labeled INTERP_CENTER, PSF_FLUX_ INTERP, indicating that interpolation over cosmic rays or bad pixels significantly affected the measured flux of the object.

3. Objects flagged in association with deblending of overlapping objects. Objects labeled as BLENDED; such objects are the parents of objects subsequently deblended into children. Objects flagged DEBLEND_TOO_MANY_ PEAKS; this flag indicates that there were more objects to deblend in this family than the deblender could handle. Objects marked PEAKS_TOO_CLOSE, indicating the peak for this object was too close to another peak.

4. Objects flagged $\mathbf{C R}$, meaning the object contained a cosmic ray that was interpolated over.

5. Objects flagged with BADSKY, meaning the estimate of the background sky at this position is suspect.

6. Objects labeled NOTCHECKED_CENTER, meaning the center of the object lies in a region not checked for peaks; often due to bad deblending.

7. Objects flagged as !STATIONARY, indicating that the object is an asteroid.

Further details of these flags are found in Stoughton et al. (2002), Richards et al. (2006), and the SDSS-III Web site.

As described in N. Padmanabhan et al. (2013, in preparation), we tracked regions of rejected objects and bad photometry to define the exact area where spectroscopy was performed. It is recommended that users apply these masks when performing statistical studies of large-scale structure with the galaxy sample or investigations that require complete understanding of the selection function, such as a measurement of the quasar luminosity function. Since the Ly $\alpha$ quasars provide random sight lines, it is unlikely that the masks will affect studies of largescale structure in the $\operatorname{Ly} \alpha$ forest.

\subsection{Galaxy Target Selection}

The dominant cosmological volume in the SDSS-I and II surveys was mapped by the LRG sample (Eisenstein et al. 2001) and the BOSS survey uses a similar philosophy of color-magnitude and color-color plots to select the galaxy sample. Unlike the LRG sample, the higher redshift BOSS ("CMASS," see below) sample is not restricted to a sample of red galaxies, but instead attempts to select a stellar mass-limited sample of objects of all intrinsic colors, with a color cut that selects almost exclusively on redshift. We lower the luminosity cut relative to the LRG sample and allow a wider color range to achieve a higher density and also provide a less biased sample for studies of massive galaxy evolution. We therefore do not 
use the designation "LRG" for these galaxies, even though their selection criteria are similar in spirit to previous LRG surveys (Eisenstein et al. 2001; Cannon et al. 2006).

The BOSS galaxies are selected to have approximately uniform comoving number density of $\bar{n}=3 \times 10^{-4} h^{3} \mathrm{Mpc}^{-3}$ out to a redshift $z=0.6$, before monotonically decreasing to zero density at $z \sim 0.8$. Galaxy shot noise and sample variance make roughly equal contributions to BAO errors when $\bar{n} P_{\mathrm{BAO}}=1$, where $P_{\mathrm{BAO}}$ is the galaxy power spectrum at the BAO scale, approximately $k=0.2 \mathrm{~h} \mathrm{Mpc}^{-1}$. For the strongly clustered galaxies observed by BOSS, $\bar{n}=3 \times 10^{-4} h^{3} \mathrm{Mpc}^{-3}$ yields roughly $\bar{n} P_{\mathrm{BAO}}=2$, making shot noise clearly subdominant. Increasing to $\bar{n} P_{\mathrm{BAO}}=2$ should also improve the performance of the reconstruction approach described in the introduction. Generating a higher space density would require substantially more observing time with only moderate gain in clustering signal-to-noise. We therefore expect significant improvements in BAO constraints over the SDSS-I and II LRG samples $\left(\bar{n}=1 \times 10^{-4} h^{3} \mathrm{Mpc}^{-3}\right)$, even in the redshift range where the two samples overlap.

The galaxy target selection algorithms will be documented in detail in N. Padmanabhan et al. (2013, in preparation); we summarize the principal points below. We select these targets from SDSS imaging according to the photometric parameters from the processing pipeline. All magnitudes are corrected for Galactic extinction using the Schlegel et al. (1998) models of dust absorption. Target galaxies are chosen based on color and apparent magnitude cuts motivated by the stellar population models of Maraston et al. (2009). These cuts are based on the expected track of a passively evolving, constant stellar mass galaxy as a function of redshift.

Targets are selected based on the following set of model magnitude colors (Eisenstein et al. 2001; Cannon et al. 2006):

$$
\begin{gathered}
c_{||}=0.7(g-r)+1.2(r-i-0.18) \\
c_{\perp}=(r-i)-(g-r) / 4-0.18 \\
d_{\perp}=(r-i)-(g-r) / 8.0 .
\end{gathered}
$$

These color combinations are designed to lie parallel or perpendicular to the locus of a passively evolving population of galaxies, with $c_{\perp}$ and $c_{\|}$being the appropriate colors to select galaxies below $z \sim 0.4$ and $d_{\perp}$ at higher redshift. To a good approximation, the perpendicular colors track the location of the $4000 \AA$ break, which is redshifted from the $g$-band to the $r$-band at $z \sim 0.4$, and provide an initial redshift selection.

Galaxy target selection mirrors the split at $z \sim 0.4$ and selects two principal samples: "LOWZ" and "CMASS." The LOWZ cut targets the redshift interval $0.15<z<0.43$. This sample includes color-selected galaxies with $16<r<19.5$, $r<13.6+c_{\|} / 0.3$, and $\left|c_{\perp}\right|<0.2$, where $r$ is the cmodel magnitude. Over most of the BOSS footprint, about one-third of the LOWZ galaxies already have spectra from SDSS-I and II; these objects are not re-observed. However, in the SGC, the SDSS-I and II spectroscopy was limited primarily to low declination, resulting in a larger fraction of LOWZ targets in the south. Cut II (CMASS for "constant mass") includes colorselected galaxies in the magnitude range $17.5<i<19.9$, $d_{\perp}>0.55$, and $i<19.86+1.6 \times\left(d_{\perp}-0.8\right)$ where $i$ is the cmodel magnitude. The CMASS sample is designed to select galaxies at $0.43<z<0.7$, although it extends beyond these nominal redshift boundaries. In addition, to better understand the completeness of the CMASS galaxies and the impact of the color-magnitude cut, we also target CMASS_SPARSE galaxies. These galaxies share the same selection as the CMASS galaxies, except that the color-magnitude cut has been shifted to $i<20.14+1.6 \times\left(d_{\perp}-0.8\right)$. We target these galaxies at a rate of $5 \mathrm{deg}^{-2}$.

In a manner similar to that described in Stoughton et al. (2002), we track the source of the selection for galaxy objects and all spectroscopic targets with flag bits encoded in the new quantities BOSS_TARGET1 and BOSS_TARGET2. Targets that satisfy the LOWZ criteria are denoted as $\mathbf{G A L} \mathbf{L O Z}$, while CMASS targets fall into several categories labeled with a GAL_CMASS_ prefix. A description of all flag bits for the main targets is found in Appendix A.

Maraston et al. (2012) present photometric stellar masses for a large sample of BOSS galaxies by fitting model spectral energy distributions to ugriz photometry. They demonstrate that the main BOSS galaxies have a narrow mass distribution that peaks at $\log \left(M / M_{\odot}\right) \sim 11.3$. The results confirm that the target selection has successfully produced a sample of galaxies with fairly uniform mass from $0.2<z<0.6$.

\subsection{Quasar Target Selection}

The primary goal of the BOSS quasar survey is to map the large-scale distribution of neutral hydrogen via absorption in the $\mathrm{Ly} \alpha$ forest. Measurements of BAO in the three-dimensional correlation function in this intergalactic neutral hydrogen will provide the first direct measurements of angular diameter distance and the Hubble parameter at redshifts $z>2$. However, for the $\operatorname{Ly} \alpha$ forest to adequately sample the three-dimensional density field for a BAO measurement, the BOSS quasar density must be an order of magnitude larger than SDSS (for the density of SDSS quasars, see Schneider et al. 2010) over the same redshift range (McDonald \& Eisenstein 2007; McQuinn $\&$ White 2011). The sample must also provide targets with a luminosity distribution that enables adequate signal-to-noise ratio spectroscopy over the forest. At a minimum, 15 quasars $\mathrm{deg}^{-2}$ at redshifts $2.15<z<3.5$ and $g_{\mathrm{PSF}}<22.0$ are required to make this measurement. Since the precision of the BAO measurement shows an approximately linear improvement with the surface density of quasars at fixed spectroscopic S/N, we attempt to obtain as many quasar sight lines as possible. Fortunately, because the quasars are nearly uncorrelated with the intervening density field, Ly $\alpha$ measurements are insensitive to the exact details of quasar target selection, and do not require the uniform sample that is essential to the galaxy BAO measurement.

Extrapolating from the Jiang et al. (2006) quasar luminosity function, we find that quasar targets must be selected to a magnitude limit of $g_{\mathrm{PSF}}<22.0$ to obtain a surface density of 15 high- $z$ quasars deg ${ }^{-2}$. However, identifying quasar targets from photometric data is complicated by photometric errors and the similarity of quasar colors (particularly at $z \sim 2.7$ ) to colors of A stars and blue horizontal branch stars (e.g., Fan 1999; Richards et al. 2002; McGreer et al. 2012). This suggests that a more sophisticated method for target selection is required than that used in SDSS-I and II (Richards et al. 2002).

In the first year of BOSS observations, we compared and tested a variety of methods to optimize the efficiency of quasar target selection. These methods included: a "Kernel Density Estimation" (Richards et al. 2009), which measures the densities of quasars and stars in color-color space from 
training sets and uses these to select high probability targets; a "Likelihood" approach which determines the likelihood that each object is a quasar, given its photometry and models for the stellar and quasar loci (Kirkpatrick et al. 2011); an "Extreme Deconvolution" (XDQSO; Bovy et al. 2011a, 2011b) selection, which performs a density estimation of stars and quasars by incorporating photometric uncertainties; and an artificial neural network (Yèche et al. 2010), which takes as input the SDSS photometry and errors from a training set in order to run a classification scheme (star versus quasar) and generate a photometric redshift estimate. XDQSO includes data from the UKIRT Infrared Deep Sky Survey (UKIDSS; Lawrence et al. 2007) and the Galaxy Evolution Explorer (Martin et al. 2005) when available, showing the greatest improvement in selection efficiency with the inclusion of the UKIDSS data.

As with galaxies, quasars targeted by BOSS are tracked with the BOSS_TARGET1 flag bits; details of each selection and flag are found in Ross et al. (2012b). Quasar target selection falls into five distinct categories.

1. QSO_CORE_MAIN, hereafter CORE, includes targets selected by a uniform method that can easily be reproduced for studies of the luminosity function, clustering, and other quasar science. The XDQSO method was selected as CORE at the beginning of the second year of BOSS observations and applies to spectroscopic plates numbered 4191-4511, and 4530 and above.

2. QSO_BONUS_MAIN, hereafter BONUS, includes targets selected in a non-uniform way to utilize the full complement of ancillary imaging data and maximize the spectroscopic quasar density. As mentioned above, heterogeneous selection does not bias cosmology constraints because the BAO measurement in the Ly $\alpha$ forest is not dependent on the properties of the background illuminating quasar.

3. QSO_KNOWN_MIDZ includes previously known $z>$ 2.15 quasars, the majority of which are from SDSS. Given the higher throughput of the BOSS spectrographs, these new observations from BOSS provide much deeper spectra in the Ly $\alpha$ forest region, and comparison to SDSS allows calibration tests and studies of spectroscopic variability.

4. QSO_FIRST_BOSS includes objects from the FIRST radio survey (Becker et al. 1995) with colors consistent with quasars at $z>2.15$.

5. QSO_KNOWN_SUPPZ includes a subset of previously known quasars (mostly from SDSS-I and II) with $1.8<$ $z<2.15$. Previous studies of the Ly $\alpha$ power spectrum (e.g., McDonald et al. 2006) indicate that metal absorption contributes a small amount of spurious clustering power in the Ly $\alpha$ forest. This lower redshift sample, re-observed with BOSS, allows a measurement of the spectral structure from metal lines that appear in the Ly $\alpha$ forest at the low-redshift range of the quasar sample, allowing the excess power to be modeled and removed from Ly $\alpha$ clustering measurements.

In addition, in the limited regions where imaging stripes overlap in the SDSS imaging survey, quasar targets were selected based on photometric variability, with color selection to choose objects at $z>2.15$. These regions cover approximately $30 \%$ of the BOSS footprint and are identified by the eighth bit in the ANCILLARY_TARGET2 flag developed for BOSS ancillary programs. This bit is referenced by name as the QSO_VAR_SDSS target flag and is found in plates numbered higher than 4953. The density of targets added by this method varies significantly from one region to another, and is on average $\sim 4 \mathrm{deg}^{-2}$. These targets lead to an increase in the density of spectroscopically confirmed quasars at $z>2.15$ of $2-3 \mathrm{deg}^{-2}$ in the overlap regions.

The CORE, BONUS, FIRST, and KNOWN samples account for an average of $20,18.5,1$, and 1.5 targets $\mathrm{deg}^{-2}$, respectively. Forty objects $\mathrm{deg}^{-2}$ are targeted as candidate quasars across the full survey (see Figures 8 and 9 in Ross et al. 2012b), although quasars were targeted at a somewhat higher density early in the survey. The QSO_KNOWN_SUPPZ and QSO_VAR_SDSS samples are used to fill unused fibers at a very low priority, resulting in a greatly varying target density. Observations in the second year included a near-final target selection for quasars and produce average densities that range from 15 to 18 quasars $\mathrm{deg}^{-2}$ at $z>2.15$, depending on the amount of ancillary imaging data (e.g., UKIDSS) and the density of stars. The selection of these targets and the target bits for the various quasar selection schemes is explained in detail in Ross et al. (2012b).

Multi-epoch imaging data in Stripe $82\left(-43^{\circ}<\alpha<\right.$ $\left.45^{\circ},-1.25<\delta<1.25\right)$ was used to determine an effective upper limit of quasar detection efficiency. By using photometric variability to select quasars, we found 24 quasars $\mathrm{deg}^{-2}$ at $z>2.15$ with $g_{\text {PSF }}<22$ (Palanque-Delabrouille et al. 2011) across the $220 \mathrm{deg}^{2}$ region. This analysis recovered quasars in the $2.5<z<3.5$ redshift range that were systematically missed by color-selection techniques because of proximity to the stellar locus and larger photometric errors in single-epoch imaging.

\subsection{Ancillary Science Targets}

As in the original SDSS spectroscopy survey, several special observing programs were designed to pursue science goals not covered by the primary galaxy and quasar targets. A total of 206 special plates were dedicated to these programs in SDSS (as described in the DR4 paper; Adelman-McCarthy et al. 2006). These SDSS plates were primarily along Stripe 82 because of the additional science goals enabled by multiple imaging epochs and because of the amount of available spectroscopic observing time in the Fall months when that region was visible. In BOSS we dedicate roughly $5 \%$ of fibers on each plate to a new series of ancillary programs. We track the selection of each object with flag bits encoded in the quantities ANCILLARY_TARGET1 and ANCILLARY_TARGET2.

These ancillary programs are intended to support studies that require sizable samples over large regions of sky, making them difficult to complete in conventional observations at shared facilities. However, because these programs are given a lower priority for target selection than the primary science drivers of BOSS, the sample selection is often not complete. These programs fall into two categories: those using the repeat and deep imaging of Stripe 82 and those selected from the rest of the BOSS imaging area. Spectroscopic observations are now complete for targets in Stripe 82 and are included in DR9. Observations of the remaining programs will continue through the end of the survey and will be included in part in DR9 and in future data releases. A description of each of the ancillary programs, including their scientific rationale, approximate density, and algorithm for target selection is found in Appendix B. Additional ancillary programs may appear in future BOSS observations; these will be documented in the corresponding SDSS Data Release papers and in the science papers that exploit them. 
Table 2

Fiber Assignment Priority for Resolving Fiber Collisions

\begin{tabular}{lll}
\hline \hline Chunks & \multicolumn{1}{c}{ Plates } & \multicolumn{1}{c}{ Target Bit Priority Order } \\
\hline boss1-boss2 & $3536-3750,4526-4529$ & quasar, galaxy \\
boss3-boss9 & $3751-4097$ & galaxy, quasar \\
boss10-boss13 & $4174-4428,4530-4556$, & QSO_FIRST_BOSS,galaxy, \\
& $4656-4669$ & QSO_KNOWN_MIDZ=CORE=BONUS \\
boss14-boss20 & $4440-4525,4557-4655$, & QSO_KNOWN_MIDZ, CORE, \\
& $4670-5140$ & QSO_FIRST_BOSS, galaxy, BONUS \\
\hline
\end{tabular}

Notes. Target types are listed in order of fiber collision priority from highest to lowest. "quasar" and "galaxy" refer to all target bits within those target classifications, i.e., all quasar or galaxy target bits have equal priority for resolving fiber collisions. A "=" symbol between two target bits means those target bits have equal collision priority. The priorities in the last row are defined for plates designed and drilled through the end of DR9 and will likely apply through the end of the BOSS survey.

\section{DESIGNING THE OBSERVATIONS}

As with the previous SDSS surveys, the BOSS spectra are obtained through observations of 1.5 radius spectroscopic plates. The SDSS-I and II plates accommodated 640 fibers (180 $\mu \mathrm{m}$ diameter) that projected to a $3^{\prime \prime}$ diameter on the sky to match the profile of the main SDSS galaxies $\left(z_{\text {mean }} \sim 0.15\right)$. Each BOSS plate contains 1000 fibers with a smaller $(120 \mu \mathrm{m})$ $2^{\prime \prime}$ diameter to reduce sky background and match the smaller angular profile of the higher redshift CMASS galaxies $\left(z_{\text {mean }} \sim\right.$ 0.57 ). The minimum allowable distance between fibers was $55^{\prime \prime}$ in SDSS and is $62^{\prime \prime}$ in BOSS, set by the cladding around each fiber. One might worry that pointing errors and seeing losses would overcome the effects of reduced sky noise for $2^{\prime \prime}$ fibers, degrading the $\mathrm{S} / \mathrm{N}$ relative to $3^{\prime \prime}$ fibers. We have conducted tests that show that we are in fact gaining on an object-by-object basis, the spectrograph throughput is significantly improved, and the increase in fiber number from 640 to 1000 (which fit in the same slithead design as SDSS) is a large efficiency gain.

Science targets are assigned to these plates in a process referred as "tiling" (Blanton et al. 2003). In SDSS-I and II, imaging and spectroscopy were interleaved, requiring the tiling to progress in pieces as the imaging for each area of the sky was completed. As explained in Section 2.1, the imaging footprint for BOSS was completed in Fall 2009, making it possible to tile the entire 10,269 $\mathrm{deg}^{2}$ footprint before full spectroscopic observations began. Changes in the target selection algorithms required occasional retilings of the survey footprint; we track changes in target selection as described below. After tiling, the plug plates are designed according to the estimated time (and thus airmass) of the observation. Target positions are converted from equatorial coordinates to $(x, y)$ plate coordinates for drilling with a Computer Numerically Controlled milling machine at the University of Washington. The application of tiling and plate design to spectroscopic observations is the same as that used during the SDSS-I and II surveys, but it was never documented. The process is fundamental to the reconstruction of the angular selection function for studies of clustering in galaxies and quasars and is described below.

\subsection{Fiber Assignment for Science Targets}

Fibers are first allocated to science targets using the tiling algorithm described in Blanton et al. (2003). The tiling process is intended to maximize the fraction of targets that are assigned fibers (tiling completeness) while minimizing the number of tiles required to complete observations (tiling efficiency) without leaving gaps of coverage in the survey footprint. The process for BOSS is nearly identical to that for SDSS-I and II, except for the number of fibers and prioritization of targets. We describe the process for BOSS here.

We begin with a uniform distribution of tiles, which are then perturbed to account for angular variations in the density of targets. Of the 1000 available fibers on each tile, a maximum of 900 fibers are allocated for science targets; the rest are reserved for calibration stars and measurements of the sky background (i.e., fibers placed at locations without detectable objects in the SDSS imaging data). Of the science fibers, five on each plate are assigned to targets that are also assigned fibers on neighboring tiles. We refer to these as "repeat observations" and use them to test the reproducibility of spectroscopic results. The remaining fibers are reserved for unique quasar, galaxy, or ancillary targets. Most plates do not use all available fibers because the plates must overlap to avoid gaps in coverage, thereby increasing the total number of plates and number of fibers beyond the minimum required by the number of science targets. The overall tiling efficiency of the survey, defined as the fraction of these 895 fibers assigned to unique, tiled science targets, is 0.927 with a plate-to-plate dispersion of 0.067 . The remaining fibers are used for lower priority targets such as additional repeat observations, QSO_VAR_SDSS targets, and additional sky fibers.

Groups of targets that are linked together within the 62" fiber collision limit are denoted as collision groups. The priorities for assigning fibers within a collision group vary with target type and have varied throughout the survey as shown in Table 2 . The decollided set of targets is the subset that does not lie within $62^{\prime \prime}$ of any other target. Targets may also be precluded from fiber assignment because they lie within a $92^{\prime \prime}$ radius of the center of each tile, which is the region covered by the centerpost of the cartridge. Given these conditions, the tiling algorithm attempts to maximize the number of targets assigned fibers. In regions covered by a single tile, fiber collisions limit the galaxy and quasar tiling completeness to $\sim 90 \%$. Once the galaxy and quasar target sets have been assigned fibers, ancillary targets are assigned to the remaining fibers. In this way, galaxy and quasar targets are never competing for fibers with the lower-priority ancillary set. Each point of the sky may be covered by up to four tiles (although overlap by more than two tiles is fairly rare). In tile overlap regions, targets within collision groups may be assigned to multiple tiles, bringing the completeness near unity in these regions (Table 3). The tiles and the imaging footprint define the geometry of the survey. We use the software package mangle (Swanson et al. 2008) to create and manipulate the survey geometry, as described briefly in Aihara et al. (2011a). 
Table 3

Completeness by Target Type and Sector Type

\begin{tabular}{llclcc}
\hline \hline Target Type & \multicolumn{2}{c}{ Single-tile Sectors } & & \multicolumn{2}{c}{ Overlapping Sectors } \\
\cline { 2 - 3 } & Mean & Dispersion $^{\mathrm{a}}$ & & Mean & Dispersion $^{\mathrm{a}}$ \\
\hline Galaxy & 0.891 & 0.010 & & 0.988 & 0.001 \\
Quasar & 0.914 & 0.030 & & 0.998 & 0.002 \\
Ancillary & 0.680 & 0.143 & & 0.850 & 0.158 \\
\hline
\end{tabular}

Note. ${ }^{a}$ rms computed from area-weighted chunk-to-chunk variation in boss 1 through boss 20 .

The fiber assignment in BOSS was not performed as a single process over the full spectroscopic footprint; instead, small regions of the footprint were tiled at different times to accommodate changes in the target selection algorithms. Each of these regions is called a "chunk" and is denoted by "bossN," where $\mathrm{N}$ is the chunk number. These chunks vary in size from a few dozen to a few hundred plates, depending on the region and the status of the target selection testing at the time the plates were needed. Within each chunk, a sector is defined as a region covered by a unique set of tiles.

While the fiber assignments changed within tiles, the tile centers were set around 2010 December in boss 15 and will not change. The final tiling of the BOSS footprint results in a total of 2208 plates as shown in Figure 1 and Table 1; 1575 plates lie in the NGC and 633 plates lie in the SGC. After accounting for spatial patterns in tiling and incomplete regions, the tiled area of the 10,269 $\mathrm{deg}^{2}$ imaging footprint (Section 2.1) amounts to a total of $10,060 \mathrm{deg}^{2}$. The mean area covered per tile is $4.7 \mathrm{deg}^{2}$ in the NGC and $4.2 \mathrm{deg}^{2}$ in the SGC. The higher tile density in the SGC is required to include approximately $20 \mathrm{deg}^{-2}$ additional galaxies from the LOWZ sample that lie in regions not covered in previous SDSS spectroscopy. The area of the survey covered by single tiles is $5417 \mathrm{deg}^{2}$, while the remaining $4643 \mathrm{deg}^{2}$ is covered by two or more tiles. The statistics of completeness for each target type are found in Table 3. Because of the connection between completeness and tile overlap, and the varying efficiency among the chunks, the incompleteness has strong spatial patterns that must be included in clustering analyses. As examples, Anderson et al. (2012) describe the method to account for incompleteness in galaxy clustering due to fiber collisions and White et al. (2012) describe the process in a measurement of quasar clustering.

\subsection{Plate Design}

In the tiling stage described above, approximately 160-200 fibers per plate are dedicated to the main quasar targets, 560-630 to galaxy targets, and 20-90 to ancillary science targets. In plate design, additional fibers are assigned to each plate for the purpose of sky subtraction and calibration.

We assign each plate at least 80 "sky" fibers placed at locations that contain no detections from the SDSS imaging survey. A random selection of such locations is output from the photometric pipeline, as described in Stoughton et al. (2002). The sky fibers are used to model the spectroscopic foreground for all science fibers. Their distribution is constrained to cover the entire focal plane, by requiring at least one sky fiber per bundle of 20 fibers. The fibers within a bundle are anchored to a fixed point on a cartridge, easing the handling of fibers but limiting the reach of a bundle. The spacing of sky fibers allows sampling of the varying sky background over the focal plane and over the optical system of the spectrograph, allowing a model to be constructed for any spatial structure and leading to improved sky subtraction.

We assign each plate 20 "standard" fibers to sources with photometric properties consistent with main-sequence F stars, to serve as spectrophotometric standards. These stars allow calibration of throughput as a function of wavelength for each exposure, including atmospheric telluric absorption corrections and the spectral response of the instrument. We choose these stars to have $15<r_{\text {fib } 2}<19$ and $m_{\text {dist }}<0.08$, where $m_{\text {dist }}$ is a scaled distance in color space from the color of a fiducial $\mathrm{F}$ star:

$$
\begin{aligned}
m_{\mathrm{dist}}= & {\left[((u-g)-0.82)^{2}+((g-r)-0.30)^{2}\right.} \\
& \left.+((r-i)-0.09)^{2}+((i-z)-0.02)^{2}\right]^{1 / 2}
\end{aligned}
$$

We use PSF magnitudes corrected for Galactic extinction to calculate this quantity. Typically there are at least $10 \mathrm{deg}^{-2}$ standard stars (or 70 per plate), although their density on the sky is a function of Galactic coordinates. These relatively hot stars provide a well understood and mostly smooth continuum that allow the spectra to be calibrated. A stellar template of appropriate temperature and surface gravity is derived for each star and used to derive the spectral response as described in D. Schlegel et al. (2013, in preparation).

During plate design, we choose 16 "guide" stars for each plate. During observations, each hole for a guide star is plugged with a coherent fiber bundle that is constructed with alignment pins to track orientation. Two guide stars near the center of the plate have large bundles $\left(30^{\prime \prime}\right.$ diameter) and are used to acquire the field. The remaining 14 guide stars use smaller bundles (10" diameter) and are used to guide during exposures. We choose guide stars from the SDSS imaging with $13<g_{\mathrm{PSF}}<$ 16.5 and colors $0.3<g-r<1.4,0<r-i<0.7$, and $-0.4<$ $i-z<1$ determined from PSF magnitudes. The positions are corrected for proper motion when data from the US Naval Observatory catalog (USNO-B; Monet et al. 2003) are available. We use the DR8 version of the SDSS astrometry both for the guide stars and the science targets. Because of errors in that astrometry, there are some offsets at declinations $\delta>+41^{\circ}$. However, as Aihara et al. (2011b) explain, these offsets are coherent on large scales, and the expected contribution of these errors on three degree scales (corresponding to the plate size) is 60 mas per star, well below our tolerances. Finally, we drill holes $(3.175 \mathrm{~mm}$, corresponding to $52^{\prime \prime} .5$ diameter) at the locations of bright stars to minimize light scattered from the surface of the plug plate. Stars brighter than $m_{V}=7.5$ are chosen from the Tycho- 2 catalog (Høg et al. 2000). These light trap holes are not plugged with fibers (they are larger size to prevent accidental plugging) and allow light to pass through the focal plane unobstructed.

The fibers assigned to standard stars, guide stars, and sky fibers are distributed uniformly over each plate to ensure consistent data quality for all spectra, regardless of their position in the focal plane. Because the fibers in the instrument are distributed in 50 bundles of 20 fibers each, we assign each hole to a particular bundle for ease of plugging. Unlike the cartridges used in SDSS-I and II, half of the BOSS fibers are colored red and half blue; the red and blue fibers alternate in position along the slit head. By tracking the holes associated with each target, we assign red fibers preferentially to CMASS targets, which are typically very faint at short wavelengths. By doing so, we minimize cross-talk on neighboring fibers inside the spectrograph between quasars and other quasars, lowredshift galaxies, or ancillary targets that have substantial blue light (since they are usually separated by at least one fiber on 
the CCD). Most bundles do not have exactly 10 high-redshift galaxies for the 10 red fibers, so the extra high-redshift galaxies are assigned to blue fibers, or the extra red fibers are assigned to other objects. Other than the bundle assignment and the $\mathrm{red} /$ blue fiber designation, there is no requirement that particular fibers be placed in particular holes. As described in Section 4.1, the exact hole where each fiber is plugged is determined shortly before observations.

In planning the survey, we estimate the Local Sidereal Time (LST) at which each plate will be observed. We use the corresponding hour angle and altitude predictions of the field during observation and determine each hole position on the plate accounting for atmospheric differential refraction (ADR). For galaxies, standard stars, or ancillary targets, we center the hole position at the optimal focus and $(x, y)$ position for $5400 \AA$ light. Since ADR depends on wavelength, redder or bluer light will be offset in $(x, y)$ coordinates away from the center of the fiber. For quasars, we center the hole position on the $4000 \AA$ light to maximize the $\mathrm{S} / \mathrm{N}$ in the Ly $\alpha$ forest. This difference corresponds to about 0.5 typically, and the overall throughput difference at wavelengths shorter than $4000 \AA$ can be about $50 \%$. In the DR9 data model (plateDesign and spAll files), the quantity LAMBDA_EFF records the wavelength for which the hole position of each object was optimized.

In addition to the wavelength-dependent ADR offset, we also account for the wavelength dependence of the focal plane when observing the quasar targets. The focal plane for $4000 \AA$ light differs in the $z$-direction from the focal plane for $5400 \AA$ light by $0-300 \mu \mathrm{m}$, depending on the distance from the center of the plate. To account for this difference, small, sticky washers are adhered to the back of the plate for quasar targets, where the fibers are plugged. The washer causes the fiber tip to sit slightly behind the $5400 \AA$ focus. No washers are used for holes within 1.02 of the plate center. Between 1.02 and $1.34,175 \mu \mathrm{m}$ washers are used, and between 1.34 and $1.49,300 \mu \mathrm{m}$ washers are used. These washers only became available after Modified Julian Date (MJD) 55441 (2010 September 2) and were not consistently used until MJD 55474 (2010 October 5). In the DR9 data model, the quantity ZOFFSET (plateDesign and spAll files) records the intended usage of washers, but not the actual usage. The exact washer usage for each observation during this transition period (including plates observed both before and after) is documented on the publicly available software Web site. ${ }^{78}$ The discrepancy will be resolved with DR10 in the summer of 2013. By optimizing the focal plane position, and thus the $\mathrm{S} / \mathrm{N}$, for $4000 \AA$ light, we are also perturbing the spectrophotometry relative to the standard stars as discussed in Section 6.1. Only the main quasar targets are optimized for $4000 \AA$ focal plane and ADR offsets in DR9. QSO_VAR_SDSS targets, and a few other programs assigned the ancillary target flags, will be similarly affected in the future. Otherwise, the plate design remains the same as it was in the SDSS-I and II surveys (Stoughton et al. 2002).

During exposures, the guider adjusts the offsets and plate scale according to changes in ADR, and adjusts rotation according to changes in altitude and azimuth. In addition, thermal expansion of the plate due to temperature changes and stellar aberration create purely radial shifts in the position of objects, these effects are corrected with changes to plate scale as predicted by the guider by adjusting the primary mirror axially and refocusing the secondary mirror. At the design hour angle, all

\footnotetext{
78 www.sdss3.org/svn/repo/idlspec2d/trunk/opfiles/washers.par
}

guide star and science target images will be centered in each fiber. However, because observations typically begin before the design hour angle and complete after the design hour angle, the image of each object will drift across the center of each fiber, and there will be no adjustment the guider can make to center all of the guide stars. To compensate, we apply changes to the plate scale to minimize the effect. The differential change in position of the image centers across the plate constrains the hour angles over which the plate is observable. We define the plate observability window such that the maximum offset of any hole relative to its image (in perfect guiding at the design wavelength, $5400 \AA$ or $4000 \AA$ ) is less than 0 '.3. The typical visibility window lasts more than $2 \mathrm{hr}$. This window is longest for plates designed to be observed at transit, and it gets progressively longer at higher declination, where the rate of change in airmass with time is slower.

\section{SPECTROSCOPIC OBSERVATIONS AND DATA REDUCTION}

The plates are designed as explained in the previous section and machined at the University of Washington months in advance of the observations. The plates are prepared by the staff at APO before observations begin. Experience from the earlier surveys motivated the procedures below to produce a survey of uniform coverage and data quality. The process of plate drilling, observing, and data processing is nearly identical to SDSS but described for the first time here.

\subsection{Plate Drilling and Preparation}

Plates are drilled at a machine shop operated by the University of Washington, where up to eight plates can be drilled in one day. The plug-plates are an aluminum alloy, $3.2 \mathrm{~mm}$ thick, $0.813 \mathrm{~m}$ in diameter, and weigh $4.3 \mathrm{~kg}$. Because the telescope focal plane is not perfectly flat, the plates are deformed during drilling to align the hole axes with the optical axes. When the plate is observed, it is similarly deformed by the cartridge to match the best-focus surface. Typical drilling position errors are $<0$ '. $15 \mathrm{rms}$, although during observations the exact angle at which the fiber rests in the hole can contribute larger errors in the focal plane position.

Plates are shipped to APO where they are received, unpacked, and "marked." In the marking stage, the original plate design is projected onto the drilled aluminum plate. Using felt-tipped markers, the staff at APO trace the groupings of 20 fibers in a bundle from the plate design onto the aluminum plate to ensure that all 1000 bundled fibers can reach all 1000 holes. Following the projected plate design, the APO staff install washers that are manufactured with an adhesive on one side around the holes of quasar fibers that need to be offset from the focal plane. They also mark the locations of holes for guide star fibers. The entire process takes around 30 minutes; an image of a marked plate is shown in Figure 2. In SDSS-I and II, plates were not observed during bright time and the staff at APO had more time to mark plates. In SDSS-III, the staff at APO are typically occupied with Marvels and APOGEE observations in bright time. We therefore typically try to complete preparation of a BOSS plate at least one month before it is observed to ease the scheduling of marking and observations. However, early commissioning plates and changes in target selection often forced last-minute shipment and plate preparation during the first year.

Once a plate is marked, a database is updated to indicate that it is available for observation. When a plate is chosen for observation on a given night based on its visibility window, 


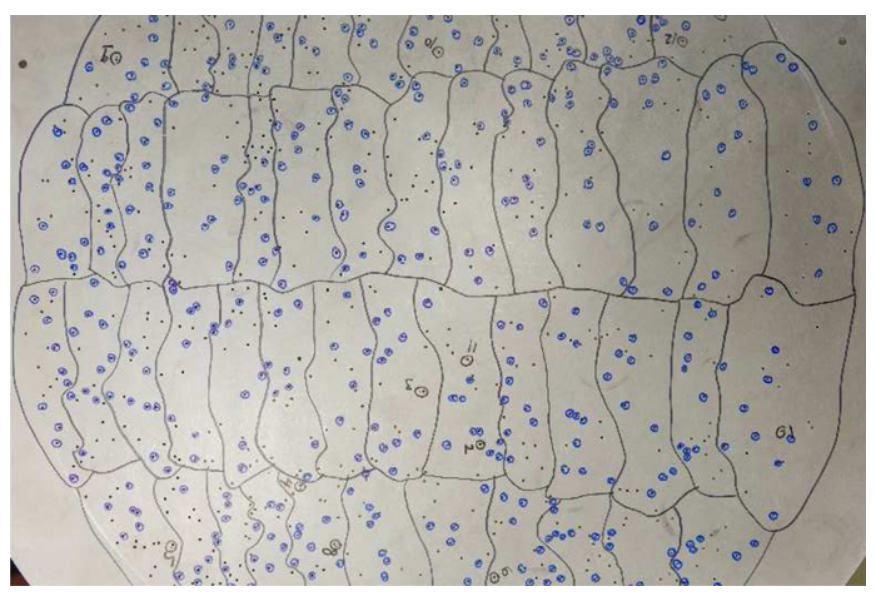

Figure 2. Image of plate 3552 immediately after the marking stage. Bundles are separated by black bounded edges, and holes are marked blue to reduce contamination between nearby emission line galaxies or quasars. Holes for guide star fibers are marked in black and denoted by the corresponding number ranging from 1 to 16 .

(A color version of this figure is available in the online journal.)

a message is sent from the nighttime staff to the daytime staff requesting that the fibers on this plate be "plugged." In the plugging stage, two staff members install the plate onto the cartridge and plug fibers from each bundle into the appropriate region of marked holes. When the plugging is complete, they measure the profile of the plate surface to confirm that the cartridge has deformed the plate to properly match the surface of the telescope focal plane. Finally, a custom-designed machine maps the location of the fibers in the focal plane by illuminating the output of each fiber with a light and recording the position of the corresponding hole with a camera that is focused on the surface of the plug plate. Repeat tests have proven the mapping process to be extremely reliable. Fibers are occasionally broken, and after plugging occasionally a fiber slips out of its hole enough to not receive light. Such cases occur $\sim 2$ times per plate, are detected during mapping, and recorded in the spectroscopic reductions. The process of plugging and mapping typically takes about 45 minutes. A full description of the marking and mapping is found in Smee et al. (2012). Up to nine BOSS cartridges can be plugged at any time.

\subsection{Procedures During Observations}

BOSS observations are performed by a rotating staff of two or three nighttime staff members known as observers. Preparations for observing begin in the afternoon; one observer checks the status of all instruments, including CCD temperatures and controller connections, followed by a bias exposure to confirm that all four detectors (two for each spectrograph) are functioning correctly. On nights when BOSS observations are planned, the afternoon observer focuses the spectrographs using a pair of neon-mercury-cadmium arc exposures behind a Hartmann mask. The Hartmann mask successively obstructs the top half and the bottom half of the collimated beam. A simple analysis of the cross-correlation between the two arc exposures reveals the optical correction required to obtain optimal spectrograph focus. The required correction to the total path length can be applied with an adjustment to the position of each collimator mirror, thereby changing the focus on the red and blue cameras by an identical amount. The afternoon adjustment confirms the performance of the instrument; the collimator mirrors are adjusted throughout the night each time observations begin for a new plate. In cases where there is a differential in focus between the two cameras, the correction is applied through adjustment of the focus ring on the blue camera, changing the path length to the blue detector with no impact on the focus on the red side of the spectrograph. Because the blue focus rings must be moved manually, they are typically only adjusted during the afternoon. Using typical nightly temperature changes and associated focus drift, the blue focus rings are adjusted every afternoon to compensate for the decrease in temperature and minimize the effect of differential focus over the course of the night. Currently, the predictive adjustment is $-4^{\circ}$ on the B1 focus ring and $+8^{\circ}$ on the B2 focus ring. Following the focus routine, a $5 \mathrm{~s}$ arc and a $30 \mathrm{~s}$ flat-field exposure are obtained to measure the spectral profile and confirm the focus of the instrument.

When the Sun is $\sim 12^{\circ}$ below the horizon in the evening, the observers fill the liquid nitrogen receptacles in all four cameras and open the telescope enclosure. The cartridge with the first scheduled plate of the night is mounted to the telescope, signaling the start of observations. A script is run to automatically slew the telescope and perform calibration routines in the following order.

1. Close the eight flat-field petals (Gunn et al. 2006) that obstruct most of the opening in the wind baffle and provide a reflective surface for the calibration lamps.

2. Turn on neon-mercury-cadmium arc lamps; they require several minutes to warm up.

3. Slew telescope to field.

4. Take a pair of exposures through the Hartmann masks using a shorter readout of the detector on a selected sub-region centered on well-known arc emission lines.

5. Adjust collimator positions to account for change in focus since afternoon checkout and cartridge change. Because focus rings are not adjusted during the night, the median between the best blue and red focus is used to adjust the collimator mirrors accordingly.

6. Acquire $5 \mathrm{~s}$ calibration arcs and $30 \mathrm{~s}$ calibration flats with a quartz lamp.

7. Open petals and take the first guider exposure through coherent fiber bundles.

When the first guider exposure is complete, the observers center the telescope on the field, using the observed guide stars. Once the guide stars are roughly centered in their respective fiber bundles, closed loop guiding begins and the plate scale of the telescope, the rotation of the telescope relative to the field, offsets in altitude and azimuth, and focus are adjusted. At this point, the light from the targeted objects is optimally focused on the fibers and the observers begin a sequence of 15 minute science exposures. When observations of the plate are completed according to the criteria described below, the telescope is moved back to the zenith, the cartridge is replaced with the next scheduled plugged cartridge, and the series of field acquisition, calibration, and exposures is repeated. The entire process takes about 15 minutes between cartridges. In the first year of the survey, approximately $65 \%$ of the available time for observing was spent with the shutter open on science targets. In the second year, we automated the scripting of field acquisition as described above and improved to $75 \%-78 \%$ efficiency, depending on the amount of exposure time required for each plate.

Ideally, we would acquire signal on each plate until the full data reductions reveal data quality that exceeds the threshold required to meet the survey requirements. However, the full 
reductions take much longer than the actual observations, so we instead use a suite of simple reductions performed in real time to provide quick feedback to the observers. We refer to these data reductions as the "Son of Spectro" (SOS) reductions, referring to the full spectroscopic reduction pipeline. At all times, a daemon automatically identifies new images as they appear on disk and submits them for flat-field calibration, wavelength solution, and the extraction of the one-dimensional spectra for each fiber. The SOS reductions perform the same functions as the spectral extraction in the full data reduction pipeline (D. Schlegel et al. 2013, in preparation), but they use simpler sky subtraction, use box-car extraction to create one-dimensional spectra, and perform no redshift or object classification. Comparing these quick reductions to the full reductions on the data, we find a reduction of approximately $20 \%$ in the SOS S/N for R1 and R2 and $10 \%$ in $\mathrm{S} / \mathrm{N}$ for B1 and B2.

Approximately 5 minutes are required to process a single flat-field exposure and 1 minute is required for arc exposures or science exposures. The results are conveyed to the observers on a Web site that automatically updates every minute. The Web site provides simple metrics for wavelength coverage, spectral resolution, profile of each fiber on the $\mathrm{CCD}$, and, most importantly, the median $\mathrm{S} / \mathrm{N}$ per pixel over a synthetic $i$-band bandpass filter for the red cameras and over a synthetic $g$-band bandpass filter for the blue cameras. Statistics of this $\mathrm{S} / \mathrm{N}$ as a function of magnitude allow diagnostics of problems in the data such as focus or guiding errors. The magnitudes are estimated from the SDSS imaging data and corrected for Galactic extinction using the maps of dust infrared emission from Schlegel et al. (1998), scaled to optical wavelengths using the model of Cardelli et al. (1989). An example of the S/N portion of the report generated for a composite of four 15 minute exposures on a single plate is shown in Figure 3.

The observers use the SOS reports to diagnose performance such as the quality of focus, the fraction of fibers that dropped from the plug plates (typically fewer than two or three on a plate), and the rate at which signal is acquired. They use the accumulated $\mathrm{S} / \mathrm{N}$ evaluated at a fiducial magnitude in the sky-dominated regime to determine when a plate is complete. As shown in the left-hand panels of Figure 3, a power law representing the $\mathrm{S} / \mathrm{N}$ as a function of magnitude is fit to determine $\mathrm{S} / \mathrm{N}$ at the fiducial values of $g_{\mathrm{fib} 2}=22$ and $i_{\mathrm{fib} 2}=21$. The $\mathrm{S} / \mathrm{N}^{2}$ scales linearly with exposure time and is therefore easier to use when estimating remaining exposure times. As explained in Section 5, the $\mathrm{S} / \mathrm{N}^{2}$ of the exposures at these magnitudes is chosen to balance high redshift completeness for the galaxy sample with the goal of observing $10,000 \mathrm{deg}^{2}$ in five years. The observers remove the cartridge when the plate is complete by the $\mathrm{S} / \mathrm{N}$ criteria and request a new plate to be plugged in that cartridge the following day. Because of limited visibility windows, it is not uncommon that observations must cease before a plate is complete. In these cases, the plate remains in the cartridge and is observed the next clear night with a new set of calibration frames. The exposures taken on all nights are considered for determining when a plate is complete.

\subsection{BOSS Data Reduction Pipeline}

At the end of each night of observing, the data are sent to LBNL to be processed. An automated software routine checks the data transfer status every 15 minutes and begins the processing when all of the data have arrived. The data processing jobs are organized by plate such that each job extracts, calibrates, co-adds, classifies, and fits the redshift of all 1000 spectra using all exposures of a single plate, including exposures taken on different nights with the same plugging of fibers. The data are reduced first by collapsing them from the two-dimensional image into one-dimensional spectra (D. Schlegel et al. 2013, in preparation). In the second step, the one-dimensional spectra are classified into object types and redshift (Bolton et al. 2012b). The software is written primarily in Interactive Data Language $(\text { IDL })^{79}$ and is collectively referred to as "idlspec2d." This processing typically begins mid-afternoon, and the results are available to the collaboration by the following morning.

Raw CCD frames are pre-processed by subtracting a bias model, their bias overscan, subtracting a dark current model, and dividing by a pixel flat-field model for each CCD. The models for bias and dark current are derived from calibration images taken periodically throughout the survey. The pixel flat-field images are computed about once every six months using a specially designed slithead that illuminates the entire detector without fiber-to-fiber spatial structure. The read noise is measured from the bias overscan region for each CCD amplifier for each exposure. Per-pixel variance is estimated using the measured read noise and the observed photon counts in each pixel. The inverse variance is multiplied by a known CCD defect mask, and cosmic rays are identified to mask affected pixels.

The spectra are extracted from the two-dimensional CCD frames into a set of one-dimensional spectra. The quartz lamp flat-field spectral images establish the cross-dispersion profile and initial location of the spectral traces as projected onto the CCDs. The locations are then shifted to match the science frame spectra to account for instrument flexure from the time of the calibration exposures to the science exposures. These offsets are typically $0.1 \AA$, but can be as large as $1.5 \AA$.

The spectra are extracted in groups of 20 according to their respective fiber bundles. We use a noise-weighted optimal extraction algorithm (Horne 1986) with the Gaussian profile cross dispersion widths measured in the flat-field spectra. A linear cross-dispersion background term is included for each bundle to account for scattered light not described by the two-dimensional Gaussian profile. The centroids and widths of the Gaussians are fixed, and the extraction solves only for the amplitudes at each wavelength and the linear background coefficients.

The wavelength solution is initially estimated from the extracted arc-lamp spectra, then shifted to match the observed sky lines in each science exposure. Since the extraction is performed in the native pixel spacing of the CCD, this results in individual spectral bins that are statistically independent but not perfectly aligned in wavelength between spectra or between exposures. The extracted science spectra from individual exposures are then divided by the extracted flat-field spectra to correct for fiber-tofiber throughput variations. Sky subtraction is performed using a model for background derived from the sky fibers that were assigned during plate design. The background varies with fiber position to account for smoothly varying differences across the focal plane. Similarly, the spectral response (i.e., the flux calibration) is determined over the focal plane using models fit to the spectra from the standard stars that were assigned in plate design.

Finally, the spectra from individual exposures are combined into a co-added frame for each fiber on a resampled grid that is linear in $\log (\lambda)$. Data from both red and blue cameras are used in the co-added frame, generating spectra that cover the full 361-1014 nm wavelength range of the instrument. The

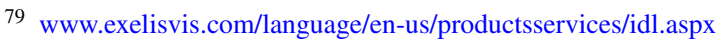



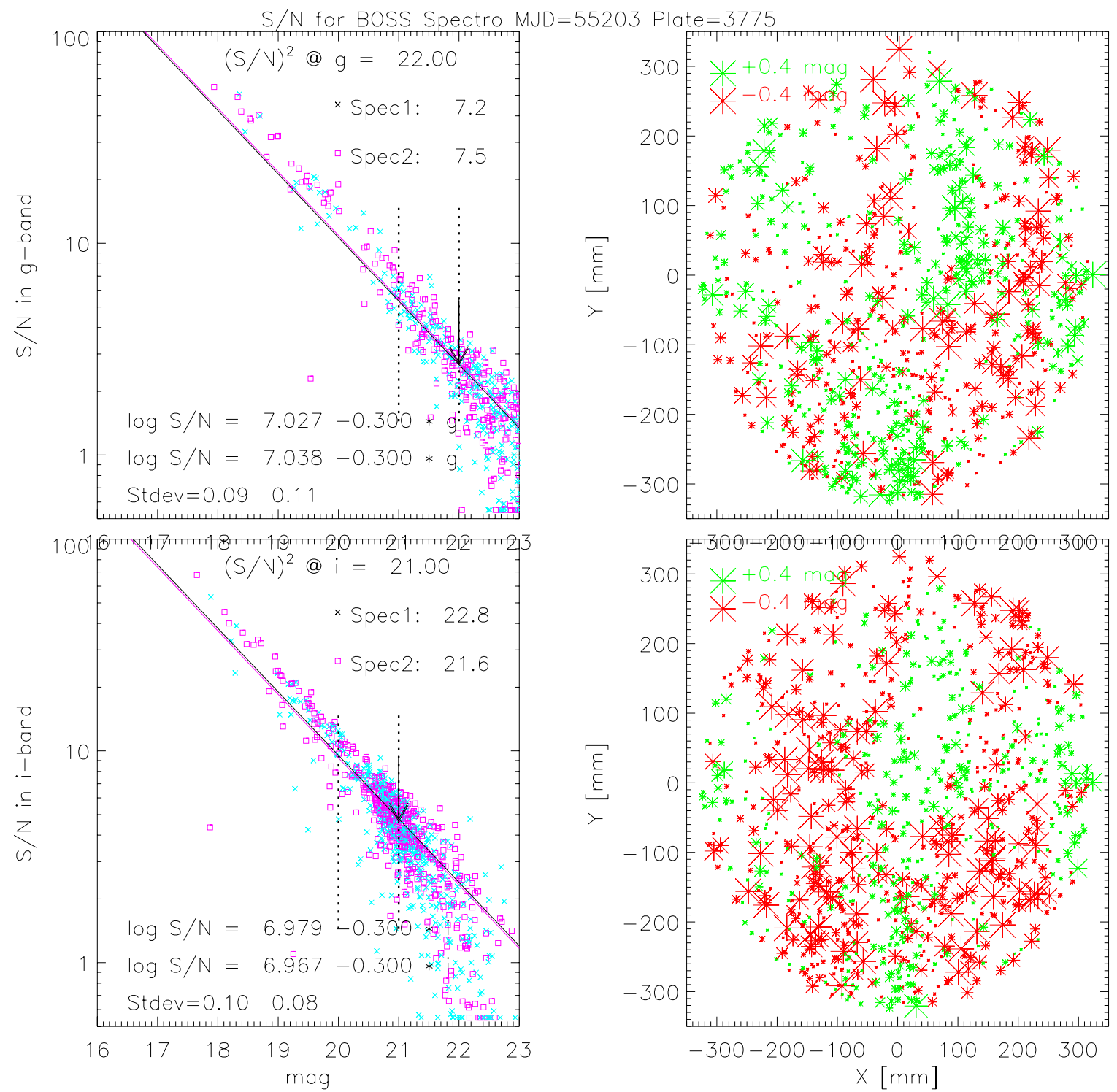

Figure 3. Example of the diagnostic from a series of four science exposures of plate 3775 that are produced in the quick reductions of data at APO. Similar plots become available to the observers less than five minutes after the end of an exposure. Left: cumulative signal-to-noise ratio ( $\mathrm{S} / \mathrm{N}$ in the figure captions) as a function of $g_{\mathrm{fib} 2}$ (top) and $i_{\mathrm{fib} 2}$ (bottom). Fibers from the two spectrographs are indicated by the blue symbol "x" (spectrograph 1) and a magenta square (spectrograph 2). The intercept of the linear fit of $\log (\mathrm{S} / \mathrm{N})$ as a function of magnitude and rms of that fit are computed for each spectrograph separately. Only fibers with fiber 2 magnitudes in the range 21-22 (20-21) for the blue (red) cameras are used in the fit as indicated by the vertical dotted lines. The slope is held fixed at -0.3 as empirically determined from fits to the larger sample. This magnitude range is near the region of sky-limited noise and near the faint end of the main galaxy and quasar samples for the red and blue cameras, respectively. Right: the spatial profile of $\mathrm{S} / \mathrm{N}$ over the plate. Red symbols represent fibers that fall below the best fit of the $\mathrm{S} / \mathrm{N}$ linear solution that is represented by the solid line in the left-hand panels. Green symbols represent fibers that fall above the best linear fit of the $\mathrm{S} / \mathrm{N}$ solution. The size of the symbol relates to the amount by which the fiber deviates, growing larger for fibers with larger deviation from the best fit. Guiding problems or other systematics can appear as coherent structure in this diagram. The distribution of objects that fall below the best-fit line is fairly uniform, indicating a lack of such effects for this particular plate. (A color version of this figure is available in the online journal.)

estimated pixel variance is propagated into variance estimates of the extracted and co-added spectra, but the covariance terms between different spectra are discarded. The current pipeline performs this extraction as a single pass, resulting in a known bias due to estimating the variance from the data rather than iteratively updating the noise model with the statistics of the extracted spectra. In the limit of zero flux, the technique systematically assigns a slightly larger variance to pixels that fluctuate toward higher flux values, leading to a weighted mean that is suppressed below its true value. This bias is not corrected in the DR9 sample, but may be addressed in a future data release. The process is performed independently for objects observed on different fibers or different pluggings of a plate, and co- added spectra are recorded for all observations. Spectra from objects that are observed multiple times are evaluated, and the observation that produces the best available unique set of spectra for the object is identified with the SPECPRIMARY flag, as described in the online documentation. ${ }^{80}$ Further details about this extraction pipeline are published in D. Schlegel et al. (2013, in preparation).

As described in Aihara et al. (2011a), star, galaxy, and quasar templates are fit to the combined one-dimensional spectra to determine the classification and redshift of each object. Redshift classification errors for all objects are reported by the

\footnotetext{
80 http://www.sdss3.org/dr8/spectro/catalogs.php
} 

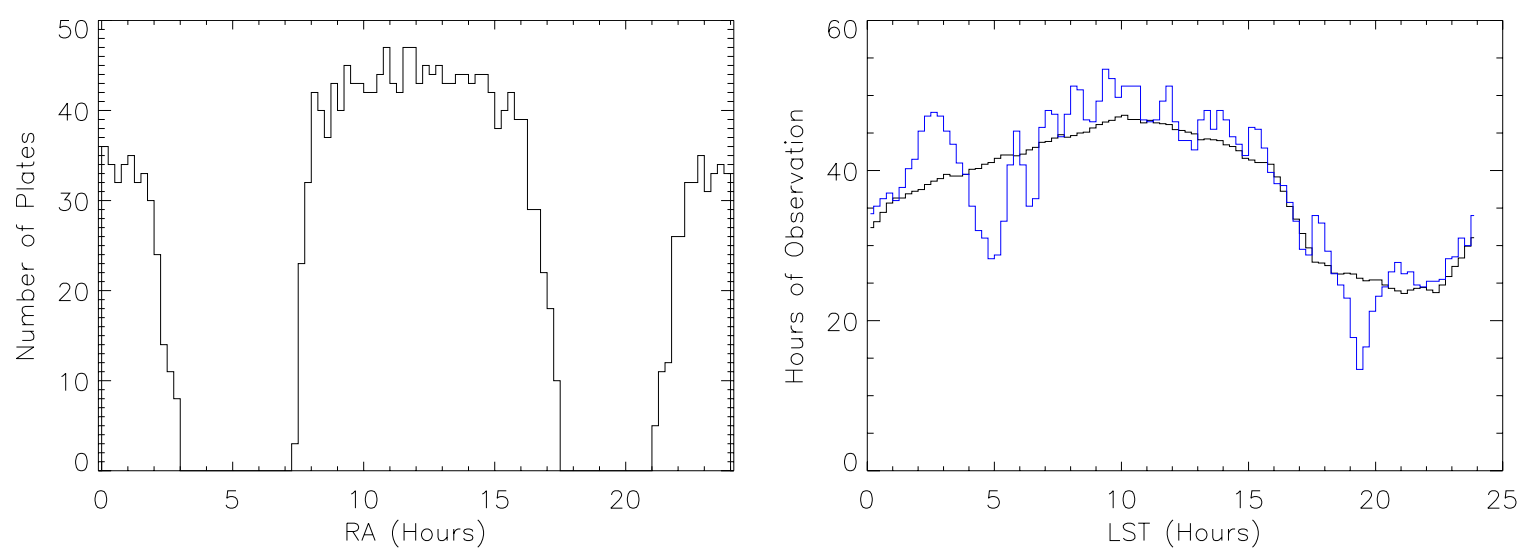

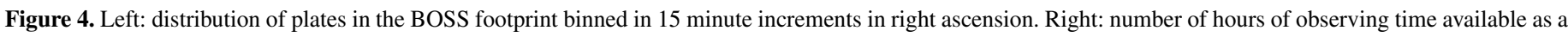

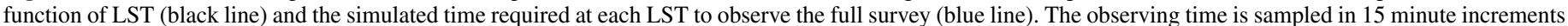

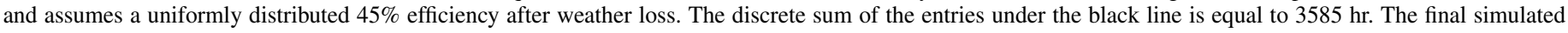
LST distribution shown in blue is discussed in Section 5.5.

(A color version of this figure is available in the online journal.)

ZWARNING bitmask keyword. We found that removing the quasar templates from the fits to the galaxy sample reduces the number of cases of catastrophic failures and classification confusion. Allowing only galaxy and stellar templates, we determine a second redshift for objects targeted as galaxies and report the redshift and classification errors with the $\mathbf{Z} \_\mathbf{N O Q S O}$ and ZWARNING_NOQSO keywords, respectively. Further details about the redshift and classification of BOSS spectra are published in Bolton et al. (2012b).

Throughout the survey, continuous improvements have been made to the spectroscopic data processing pipeline. Every few months a new version is tagged and all of the data are reprocessed starting from the raw data. These tagged versions are released to the collaboration for internal use and the daily processing continues with the latest internally released tag. The majority of processing time is spent on redshift and object classification due to the large range of redshift over which templates must be fit. The total processing takes 8-12 hr per plate, although batch jobs allow an entire data set to be processed in a few days. Version v5_4_45 of the idlspec $2 \mathrm{~d}$ pipeline code is used to report the results from DR9.

Data reduction results are mirrored to New York University (NYU) on a daily basis to serve as an offsite backup. In addition, the daily reductions are processed in parallel on a Linux cluster at the University of Utah (UU), thus ensuring continuously tested alternate processing capability. The standard data flow is from APO to LBNL to NYU and UU; approximately once a year alternate paths are tested (e.g., APO to NYU to LBNL and UU). Disaster recovery plans with a $24-48$ hr turnaround have been developed and tested, with scenarios ranging from the outage of a single disk to permanent loss of the LBNL computing center.

DR9 includes all of the spectral products available to the BOSS collaboration: co-added and individual exposure flux calibrated spectra, inverse variances per pixel, masks, subtracted sky, calibration vectors, and model fits. Intermediate data products such as uncalibrated spectra and extracted arc/flat lamp spectra are also available. Catalog data such as redshifts, classifications, astrometry, and quality flags are available via the SDSS-III Catalog Archive Server database and in a fits file binary table (spAll-v5_4_45.fits). The spectra themselves are available in a format of one plate-mjd-fiber per file, or in a bundled format of all spectra of all objects for each plate-mjd.
Details for accessing these data are at http://www.sdss3.org/dr9/ and Ahn et al. (2012).

\section{PROJECTIONS FOR COMPLETING THE SURVEY}

BOSS has been allocated the majority of the dark and gray time in the SDSS-III survey since Fall 2009. Bright time and a fraction of gray time are used for high resolution spectroscopy of Milky Way stars to search for extrasolar planets (MARVELS; Ge et al. 2009) and to measure abundances in evolved, latetype stars from $H$-band spectroscopy to study evolution of the Milky Way galaxy (APOGEE; S. R. Majewski et al. 2013, in preparation). These programs are less sensitive to the increased sky background. On nights when the moon is illuminated at less than $39 \%$, all of the observing time is allocated to BOSS. On nights when the moon is illuminated at more than $56 \%$, time is given to BOSS when the moon is below the horizon. For nights when the moon is between $39 \%$ and $56 \%$ illumination, the allocation of time depends on the time of year. In these phases, BOSS is only given time when the moon is below the horizon when the NGC cannot be observed; BOSS is given the full nights when the NGC can be observed. Time is only split between projects on a given night if there are at least $1.4 \mathrm{hr}$ allocated to each survey.

Based on historical records, we assume that $55 \%$ of the time will be lost to bad weather and that most of the months of July and August will be lost to summer monsoon. Taking the total amount of time allocated to BOSS and the expected weather loss, we anticipate approximately $3600 \mathrm{hr}$ of observation over the course of the survey from 2009 December through 2014 July. The distribution of this time as a function of LST is shown in Figure 4. To maximize the survey volume to probe BAO with galaxies and $\mathrm{Ly} \alpha$ quasars, our goal is to observe the entire 10,060 $\mathrm{deg}^{2}$ footprint tiled with targets from the SDSS imaging program. Given the total number of spectroscopic plates over this area, and assuming perfect scheduling, the average target can be observed for $1.62 \mathrm{hr}$ including overhead due to field acquisition and calibration exposures. It is essential to characterize the on-sky performance of BOSS to maximize the efficiency of these integrations over the full five-year survey.

A comparison of the left- and right-hand panels of Figure 4 quickly reveals that the distribution of plates in right ascension is not perfectly matched to the time available to BOSS over the 
Table 4

Spectroscopic Classification in Subsets of First Year Data

\begin{tabular}{|c|c|c|}
\hline Data Subsample & $\mathrm{S} / \mathrm{N}_{i}^{2} \geqslant 20 \mathrm{AND} \mathrm{S} / \mathrm{N}_{g}^{2} \geqslant 10$ & $\mathrm{~S} / \mathrm{N}_{i}^{2}<20 \mathrm{OR} \mathrm{S} / \mathrm{N}_{g}^{2}<10$ \\
\hline LOWZ & 0.995 & 0.992 \\
\hline CMASS & 0.942 & 0.920 \\
\hline CMASS $\left(i_{\text {fib2 }}<21.5\right)$ & 0.966 & 0.955 \\
\hline CMASS $\left(21.5<i_{\text {fib } 2}<21.7\right)$ & 0.851 & 0.741 \\
\hline CMASS $\left(i_{\text {fib } 2}>21.7\right)$ & 0.634 & 0.512 \\
\hline
\end{tabular}

course of the survey. The differences are particularly large in the intervals $3 \mathrm{hr}<\mathrm{LST}<7 \mathrm{hr}$ and $17.5 \mathrm{hr}<\mathrm{LST}<21 \mathrm{hr}$, when the Galactic plane passes directly overhead. We must then observe the high Galactic latitude BOSS plates at these times at non-zero hour angles. Hour angles are chosen to optimize LST coverage while minimizing the additional observation time required to account for the higher airmass.

Observations are planned at a high target density to measure the BAO signal, obtain high redshift completeness, and finish the survey (Figure 1) in the allotted time. We quantify redshift completeness in the context of the value of $\mathrm{S} / \mathrm{N}^{2}$ reported by the SOS reductions and find that exposures must be at least as deep as $\mathrm{S} / \mathrm{N}^{2}>20$ on the red cameras and $\mathrm{S} / \mathrm{N}^{2}>10$ on the blue cameras to obtain the desired completeness in galaxy redshifts. We estimate the maximum exposure time that allows completion of the full BOSS footprint and find that we have time to allow for slightly deeper exposures than that minimum requirement. In this section, we describe the tension between these two constraints and our analysis to determine the optimal hour angles and $\mathrm{S} / \mathrm{N}^{2}$ thresholds.

\subsection{Galaxy Redshift Completeness}

Meeting the projections for BAO constraints from the galaxy sample described in Section 2.3 requires that we measure galaxy redshifts for $>94 \%$ of the targets, where the remainder are either not galaxies or are recognized redshift-fitting failures. For comparison, the SDSS main and LRG spectroscopic samples had a redshift success rate of $>99 \%$ for galaxies at $0<z<0.45$ (Strauss et al. 2002). We first identify an $\mathrm{S} / \mathrm{N}^{2}$ threshold at the fiducial magnitude for determining the completion of a plate that realizes this $94 \%$ redshift success rate for galaxies.

In the first year of BOSS operations, we lacked the information to empirically determine the quality of a typical exposure or the depth of the data required to obtain redshifts for faint targets. We thus intentionally chose a threshold that produced deeper data than would allow us to finish the survey in the five year window, with the idea of adjusting the thresholds once redshift success rates could be quantified. Specifically, we chose a threshold of $\mathrm{S} / \mathrm{N}^{2}>16$ in the blue cameras evaluated at $g_{\text {fib2 }}=22$, and $\mathrm{S} / \mathrm{N}^{2}>26$ for the red cameras evaluated at $i_{\text {fib2 }}=21$. For nights when the plate visibility window expired before meeting these thresholds, a plate was considered complete if it reached $\mathrm{S} / \mathrm{N}^{2}>13$ and $\mathrm{S} / \mathrm{N}^{2}>22$ on the blue and red cameras, respectively. Plates that did not reach these thresholds were kept in their cartridges and observed on the next clear night.

To quantify the minimum $\mathrm{S} / \mathrm{N}^{2}$ to reach the survey goals, observations from the first year were artificially degraded by removing $33 \%$ of the 15 minute exposures on each plate. Exposure depths for each plate were determined from the remaining subset of exposures using the SOS reductions. The exposures were combined as explained in Section 4.3 and evaluated for redshift completeness. A successful classification of an LOWZ or CMASS galaxy target is one that produces ZWARNING_NOQSO $=0$. A reduction in redshift completeness becomes evident at $\mathrm{S} / \mathrm{N}^{2} \leqslant 20$ at the fiducial magnitude on the red cameras and $S / N^{2} \leqslant 10$ on the blue cameras. The redshift completeness of the LOWZ sample and the brightest objects in the CMASS sample are only marginally impacted by the reduced $\mathrm{S} / \mathrm{N}^{2}$, but the faintest objects in the CMASS sample $\left(i_{\mathrm{fib} 2}>21.5\right)$ are quite sensitive to the $\mathrm{S} / \mathrm{N}^{2}$.

The results imply that each exposure must satisfy $S / N^{2} \geqslant 20$ for the red cameras and $\mathrm{S} / \mathrm{N}^{2} \geqslant 10$ for the blue cameras to obtain a reliable classification of the CMASS objects. These $\mathrm{S} / \mathrm{N}^{2}$ values define the absolute minimum threshold that can be used, beginning in the second year when the observing procedures were updated. Table 4 shows the impacts of applying these thresholds on the redshift success rate for various subsets of the data. When applying this minimum $\mathrm{S} / \mathrm{N}$ threshold, we find that $94 \%$ of the CMASS sample is classified with ZWARNING_NOQSO $=0$. However, less than $65 \%$ of the subset of CMASS targets with $i_{\text {fib2 }}>21.7$ are classified successfully and only $85 \%$ of the subset of CMASS targets with $21.5<i_{\text {fib } 2}<21.7$ are classified successfully. The lower efficiency for targets with $i_{\text {fib2 }}>21.5$ led to the decision to remove these targets from the galaxy target selection after the first year, effectively reducing the size of the CMASS sample by $5.2 \%$. The remaining CMASS targets should be classified at an efficiency greater than $96 \%$ as long as all plates are observed to a depth $\mathrm{S} / \mathrm{N}_{i}^{2} \geqslant 20$ and $\mathrm{S} / \mathrm{N}_{g}^{2} \geqslant 10$.

These completeness estimates are based on the current spectroscopic data reduction, which does a good but not perfect job of spectral extraction and classification. We are continuing an effort to implement the "spectro-perfectionism" spectral extraction scheme (Bolton \& Schlegel 2010) and to develop new templates for classifying galaxies and quasars. We anticipate that the redshift completeness will ultimately exceed the performance in DR9. Under good observing conditions at low airmass and low Galactic extinction, the $\mathrm{S} / \mathrm{N}_{i}^{2} \geqslant 20$ condition is more demanding than the $S / N_{g}^{2} \geqslant 10$ condition. However, if the blue criterion is more stringent, then some plates at high airmass or low Galactic latitude take a very long time to complete. Figure 5 shows the distribution of $\mathrm{S} / \mathrm{N}_{i}^{2}$ and $\mathrm{S} / \mathrm{N}_{g}^{2}$ in BOSS observations taken in the first year. Note that the $\mathrm{S} / \mathrm{N}_{i}^{2}$ typically exceeds the threshold $\left(\mathrm{S} / \mathrm{N}_{i}^{2}>26\right.$ for year one) by a small margin because exposures are taken in 15 minute intervals, even when the plate is close to threshold. Some plates are well above threshold because we occasionally had too few plates available for a given LST and therefore had to "overcook" the plates that we had.

\subsection{Quasar Identification and Ly $\alpha$ Forest $S / N$}

While the performance of the galaxy component of BOSS is easily captured by the redshift efficiency, the performance of the quasar component is complicated by the fact that the entire Ly $\alpha$ forest region of each quasar spectrum is important. While 


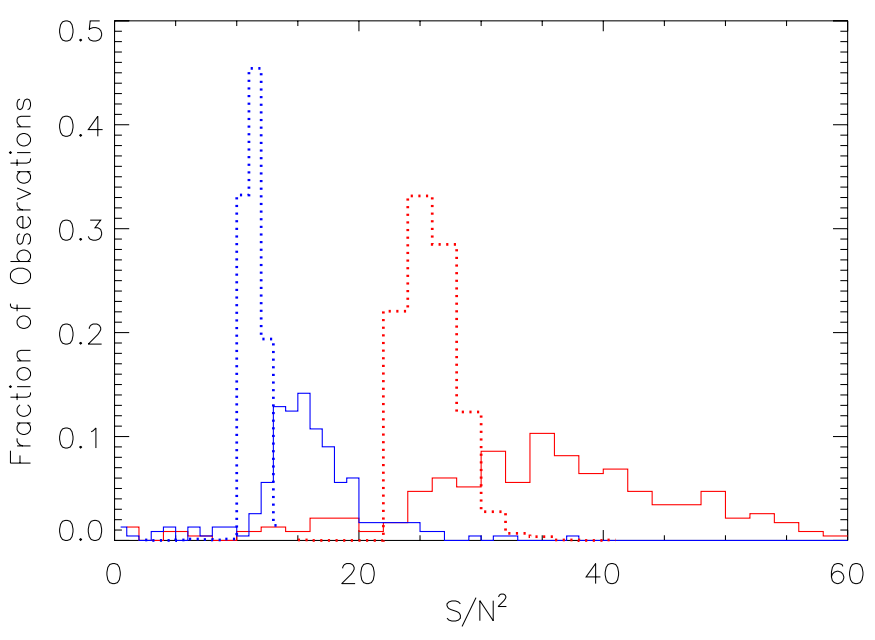

Figure 5. Fraction of completed plates as a function of $S / N^{2}$ as reported by SOS. The $\mathrm{S} / \mathrm{N}^{2}$ for the first year of data is shown as a solid line while the $\mathrm{S} / \mathrm{N}^{2}$ for the simulated survey, with somewhat lower thresholds as described in Section 5.5, is shown as the broken line. In both cases, the $S / \mathrm{N}^{2}$ for the red cameras is shown in red while the $\mathrm{S} / \mathrm{N}^{2}$ for blue cameras is shown in blue.

(A color version of this figure is available in the online journal.)

an increase in exposure times leads to deeper spectra in the Ly $\alpha$ forest, the extra time also reduces the total sky coverage. When we considered changing the exposure depths after the first year of BOSS, scaling arguments based on McDonald \& Eisenstein (2007) indicated that for BAO studies, the gains from additional area would exceed the losses from lower $\mathrm{S} / \mathrm{N}$ in the Ly $\alpha$ forest. In a more thorough recent investigation, Font-Ribera et al. (2012) address the question using detailed simulated Ly $\alpha$ forest catalogs constructed from Gaussian random density field realizations. They simulate quasars with a density $15-17 \mathrm{deg}^{-2}$, $g<22$, exposure depths comparable to those in BOSS, and a redshift $2.15<z<3.5$. Noise is added to each quasar spectrum assuming typical sky and readout noise from BOSS. They find that the errors on the Ly $\alpha$ forest correlation function increase by $10 \%-15 \%$ if the exposure times are halved, and conversely, that those errors would be reduced by $30 \%$ in the limit of zero noise. Because the error bars on the correlation function scale as the inverse square root of survey area, the simulations confirm a decision to favor area over depth in the Ly $\alpha$ spectra. We report the quality of the BOSS Ly $\alpha$ spectra in Section 6.4 in comparison to expectations from theoretical predictions for an optimal quasar survey.

Investigating the large-scale structure of $\operatorname{Ly} \alpha$ absorption relies critically on our ability to classify quasars and determine their redshifts in the first place. Quasars at $z>2.15$ are easily identified due to strong emission lines such as $\mathrm{Mg}$ II $\lambda 2798$, C III $\lambda 1908$, C IV $\lambda 1549$, and Ly $\alpha \lambda 1215$. As discussed in Pâris et al. (2012), the typical central pixel for a $g_{\mathrm{PSF}} \sim 22 \mathrm{DR} 9$ quasar in these emission line regions has an $\mathrm{S} / \mathrm{N}=0.62, \mathrm{~S} / \mathrm{N}=1.4$, $\mathrm{S} / \mathrm{N}=3.0$, and $\mathrm{S} / \mathrm{N}=4.3$, respectively. For comparison, the median $\mathrm{S} / \mathrm{N}$ per pixel over the whole spectrum at $g_{\mathrm{PSF}} \sim 22$ is 0.90 . In many spectra of quasars at $g_{\mathrm{PSF}} \sim 22$, only Ly $\alpha$ and C IV emission lines are used in identification; about half of quasar targets are confirmed to be at $z>2.15$. While incompleteness is substantial, it is important to emphasize that the vast majority of objects that are not confirmed Ly $\alpha$ quasars are likely to be stars, which lack the strong emission features of typical quasars. Some of the failures may also be BL Lac objects or other weaklined active galactic nuclei (AGNs). In any event, the level of spectroscopic incompleteness does not seriously impact our ability to measure structure in the Ly $\alpha$ forest.

As an additional test of the spectroscopic completeness of the quasar sample, we performed spectroscopy on seven plates with quasars selected to $g_{\text {PSF }}<22.5$, half a magnitude fainter than the typical limit for the main quasar sample. These targets fall on plates 5141-5147 and were chosen based on variability without the color selection that was imposed on the other variabilityselected targets in Stripe 82. Comparing shallow exposures of plate 5141 (the only one of these plates included in DR9) to deeper exposures, we found a redshift success rate $\sim 100 \%$ for quasar targets with $g_{\mathrm{PSF}}<22$ and $\sim 80 \%$ for $22<g<22.5$. A manual classification revealed that no objects were falsely classified as quasars in the shorter exposures. While the quality of the quasar spectra is clearly an essential feature of the BOSS survey, we conclude that even shallow BOSS exposures are adequate to reliably classify quasars and that survey area is more important than depth for signal in the Ly $\alpha$ forest region. We therefore base our metric for exposure depths entirely on the redshift efficiency for the galaxy sample.

\subsection{Atmospheric and Galactic Extinction}

Designing the survey, scheduling plates for plugging and observation, and tracking survey progress against completion of the full footprint require that we have accurate predictions for the total exposure time that will be needed for each plate to reach at least the $\mathrm{S} / \mathrm{N}$ thresholds given in Section 5.1. In order to determine how airmass effects $S / \mathrm{N}^{2}$, we first predict the impact of observing conditions on the depth of the exposures. While the depth of each exposure is affected by seeing conditions, changes in atmospheric extinction, sky brightness, and cloud cover, these processes are stochastic and cannot be predicted for a given plate at the time of observation. On the other hand, airmass and Galactic extinction only depend on the plate coordinates and design hour angle. We used the data from the first year of BOSS observations to understand their effect on the mean $\mathrm{S} / \mathrm{N}^{2}$.

Using the reported $\mathrm{S} / \mathrm{N}^{2}$ for each exposure in the first year from the SOS data reductions, we found that the scaling of the mean $\mathrm{S} / \mathrm{N}^{2}$ with Galactic extinction is well described by the expected relationship:

$$
\mathrm{S} / \mathrm{N}^{2}=\text { constant } \times 10^{-2 A_{X} / 2.5},
$$

where $A_{X}$ is the predicted extinction from Schlegel et al. (1998) in magnitudes in the synthetic bandpass filter " $X$." We then quantify the effect of airmass on the $S / N^{2}$ from the SOS reductions. Higher airmass degrades the $\mathrm{S} / \mathrm{N}$ by introducing additional sky background, increasing the seeing, and reducing atmospheric transparency. The exposures are binned by airmass and averaged in each bin to account for all other weather effects. We find a power-law dependence on airmass $(Y)$; the mean $\mathrm{S} / \mathrm{N}^{2}$ for a single exposure at the fiducial magnitudes scales for the red and blue cameras as

$$
\begin{aligned}
& \mathrm{S} / \mathrm{N}_{i}^{2}=7.5 \times Y^{-1.25} \\
& \mathrm{~S} / \mathrm{N}_{g}^{2}=3.6 \times Y^{-1.0} .
\end{aligned}
$$

These relations accurately describe the data up to an airmass of 1.3. Beyond that limit, the data become roughly independent of airmass with substantial scatter. 


\subsection{Determining Plate Hour Angles}

We attempt to assign the range of LST that each spectroscopic plug plate can be observed in a manner that covers the available time allocation for BOSS with the highest $\mathrm{S} / \mathrm{N}$ for the whole survey. We calculate the Galactic extinction at the center of each tile. We then determine the hour angle and corresponding airmass at each available timeslot shown in the right-hand panel of Figure 4 for that tile. At this stage, we impose several constraints on the observations. Tiles within $\delta \pm 5^{\circ}$ of the APO latitude of $+32^{\circ} 47^{\prime}$ must be designed at $|\mathrm{HA}| \geqslant 1 \mathrm{hr}$ to prevent zenith crossing, where telescope tracking in altitude-azimuth becomes uncertain. We then define a maximum hour angle for each tile to ensure that plates are not observed at an airmass that would lead to visibility windows that are significantly less than $2 \mathrm{hr}$. Finally, to simplify plate design, we assign hour angles in increments of 20 minutes. All plates are limited to a design hour angle of less than $\pm 3^{\mathrm{h}} 20^{\mathrm{m}}$.

Using the models for dependence on airmass and Galactic extinction in Section 5.3, we estimate the effective exposure time required to complete each plate at each timeslot. We define the weight of each plate as the fractional increase in exposure time required to achieve an $\mathrm{S} / \mathrm{N}^{2}$ identical to a plate at zero Galactic extinction, airmass unity, mean seeing conditions, and mean atmospheric conditions. We then rank the plates in order of increasing weight at each LST and determine the most expensive timeslots to perform observations. The regions that are the most expensive are those that have the highest weight for the topranked plates. These times occur during the Galactic plane crossing at $\sim 4.6 \mathrm{hr}$ and $\sim 20 \mathrm{hr}$ LST, where the fields tend to be observed at high airmass.

Given the celestial boundaries in the survey, we assign hour angles to plates in the NGC and SGC independently. We start with plates in the NGC with the timeslot at $4.6 \mathrm{hr}$ LST and the $N$ highest ranked plates for the $N$ days that are predicted to have observations at that LST. We simulate observations of each plate using an integral number of 15 minute exposures, a 75\% observing efficiency, and an $\mathrm{S} / \mathrm{N}$ that evolves according to the change in airmass over the course of the observation. The typical simulated observation of a plate with $\alpha \sim 7.5 \mathrm{hr}$ takes between 1.5 and $2 \mathrm{hr}$. It is these plates that are observed at high hour angle around 4.6-6 hr LST. We subtract the integrated exposure time for each plate over the range of LST in the same manner as actual observations would take place. We similarly simulate observations for the plates with $\alpha \sim 17 \mathrm{hr}$ that are needed for observation at the $20 \mathrm{hr}$ LST timeslot. We alternate between the eastern and western edges of the NGC, incrementally using plates closer to the center of the NGC as the LST approaches $12-13 \mathrm{hr}$, until all of the available time is used. We then assign hour angles to plates in the SGC following the same technique.

\subsection{Determining Exposure Depths and Updating Plate Designs}

We next evaluate the amount of time required to complete the plates to determine how much additional signal beyond the minimum thresholds $\left(\mathrm{S} / \mathrm{N}_{g}^{2}>10, \mathrm{~S} / \mathrm{N}_{i}^{2}>20\right)$ can be acquired while still completing the survey. The process of hour angle assignment is performed iteratively by varying the minimum depth of each exposure and varying the LST range covered by the NGC and SGC plates. Simulated 15 minute exposures are accumulated until the $\mathrm{S} / \mathrm{N}^{2}$ exceeds some minimum threshold as described in Section 4.2. We manually vary the thresholds of both the blue and red cameras until the simulations produce a completed survey in the amount of time allocated to BOSS. We adjust the LST times that divide plates between the NGC and SGC until both regions are observed in the amount of time allocated; plates from the NGC should be observed between an LST of $4.9 \mathrm{hr}$ and an LST of $19.1 \mathrm{hr}$ while plates from the SGC should be observed at other times.

We find that we can complete the survey if we set an $\mathrm{S} / \mathrm{N}^{2}$ threshold of 22 pixel $^{-1}$ for the synthetic $i$-band filter and 10 pixel $^{-1}$ for the synthetic $g$-band filter at their respective fiducial fiber2 magnitudes. As argued in Section 5.1, this depth is sufficient to accurately obtain redshifts for the vast majority of CMASS galaxy targets. The mean exposure time for the survey will be $1.61 \mathrm{hr}$ per plate, amounting to a total of $3551 \mathrm{hr}$ of exposure time. Because the plates in the NGC are located at a higher declination on average, and in regions that have smaller amounts of Galactic dust, the typical plate in the NGC will be completed in $1.49 \mathrm{hr}$, while the average plate in the SGC will be completed in $1.92 \mathrm{hr}$. The projected time spent observing at each LST is shown in Figure 4 and a histogram of predicted plate $\mathrm{S} / \mathrm{N}^{2}$ for the full survey is shown in Figure 5.

Figure 4 reveals two additional features of the BOSS survey projections. The first feature is a minor shortage of plates in the LST regions covered by the Galactic plane, appearing as dips around 5 and $20 \mathrm{hr}$. It is during these times that the observations are affected by the limitation on hour angles mentioned above. This shortage of plates was actually exacerbated during the first two years of observations. The second feature appears as an excess of plates between 21 and $3 \mathrm{hr}$ LST. This is the part of the SGC that is visible in the summer months and early Fall when nights are short and when the telescope is closed for six weeks due to yearly maintenance. It is likely that BOSS will have unobserved plates in this region when the survey is complete. To minimize similar gaps at other ranges of LST, we update the survey projections every few months to account for the amount of time remaining in the survey and the plates completed.

\subsection{Maintaining a High Observing Efficiency}

We require a high observing efficiency to accomplish the goal of completing the survey footprint within a fixed time window that is set largely by funding constraints. To do so, we balance nightly observing efficiency against the higher priority of protecting the telescope and instruments to avoid down time or catastrophic damage. Over the years since the beginning of SDSS, we have adopted a number of strategies to achieve this balance.

We assign two night-time observers at all times as discussed in Section 4.2. Two observers are scheduled mainly for observing efficiency and safety. The "warm" observer is responsible for setting up the software that controls the telescope and instruments, starting and monitoring data collection, checking SOS feedback, and other tasks that can be performed indoors. The "cold" observer's duties include swapping cartridges, checking data quality, and writing the night log. To minimize the amount of time between cartridge changes, the cold observer prepares for the change before the final exposure is completed. As soon as the final exposure is complete, the warm observer moves the telescope to zenith and the cold observer physically locks the system so that the telescope cannot move. As soon as the cold observer mounts the new cartridge to the telescope, the warm observer updates the software control system to reflect the cartridge change. The cold observer, still outdoors, releases the telescope so that slewing can begin. With two observers on duty, we shorten each cartridge change by between five and six 


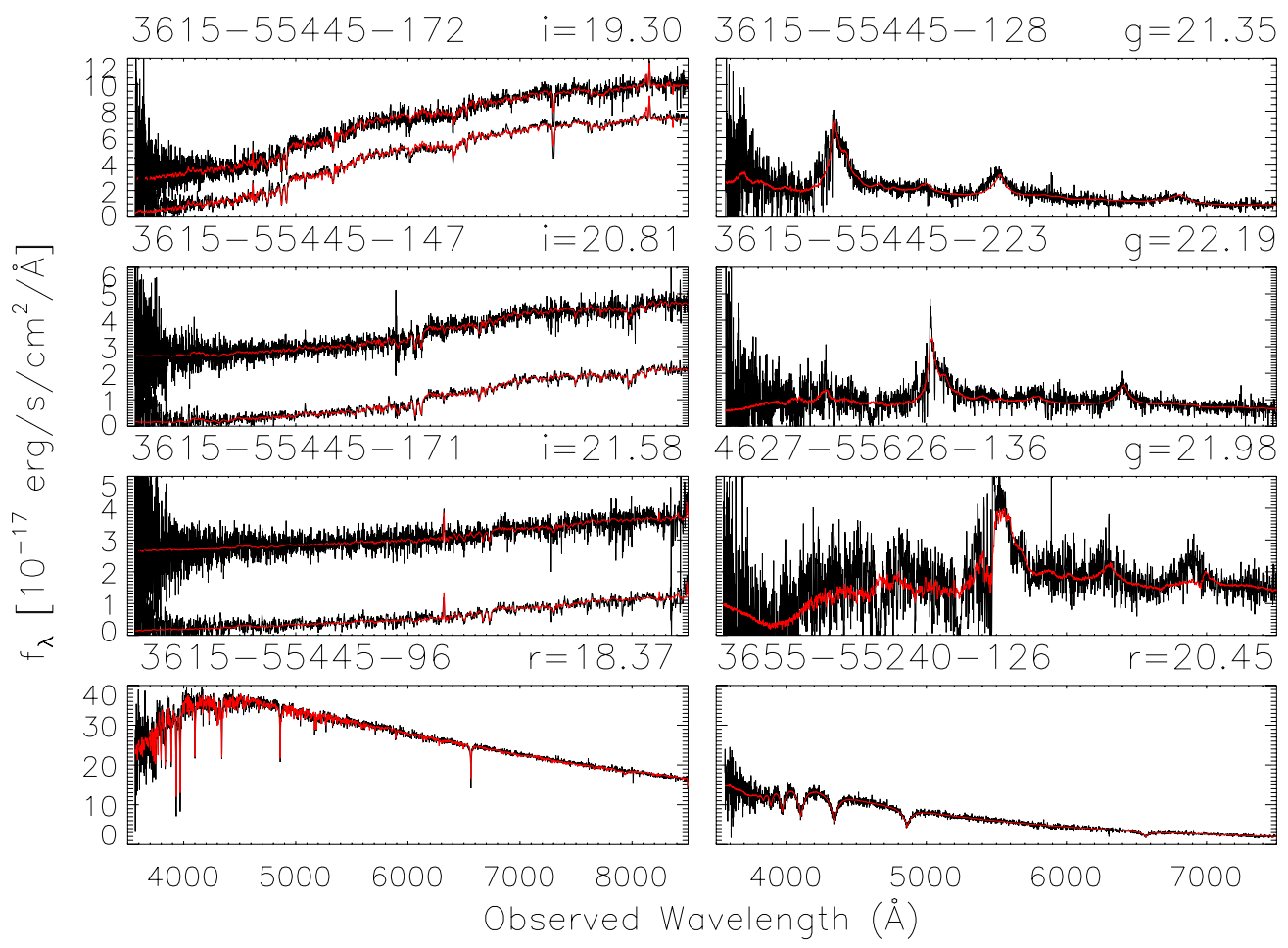

Figure 6. Mosaic of BOSS spectra (black) and best-fit idlspec2d templates (red) of varying luminosity. The plate, MJD, fiber, and fiber2 magnitude are listed at the top of each panel. Left (top three panels): Galaxy spectra from the LOWZ and CMASS samples in order of decreasing luminosity and increasing redshift: a $z=0.238$ LOWZ galaxy, a $z=0.541$ CMASS galaxy, and a $z=0.695$ CMASS galaxy that is slightly fainter than the $i_{\text {fib } 2}<21.5$ threshold that was imposed after year one. In each case, the top spectrum is offset by $2.5 \times 10^{-17} \mathrm{erg} \mathrm{s}^{-1} \mathrm{~cm}^{-2} \AA^{-1}$ and the bottom spectrum is smoothed with a five-pixel median boxcar filter. Right (top three panels): unsmoothed quasar spectra from the CORE and BONUS samples in order of increasing redshift: a $z=2.57$ quasar, a $z=3.14$ quasar, and a $z=3.53$ quasar. Bottom: unsmoothed spectrum of a standard star (left) and of a white dwarf star (right).

(A color version of this figure is available in the online journal.)

minutes (saving 2-4 exposures per night), ensure better data quality, and guarantee that all parts of the system are functioning correctly. Operations safety is another important reason for scheduling two observers. While the cold observer is performing duties outside, the warm observer is monitoring the process from a camera that feeds to video in the control room. Therefore, the second observer can respond quickly in the unlikely case of an accident. All observers are trained to perform both warm and cold roles to ensure continuity between observing shift changes.

Both observers monitor the weather conditions and determine when to close the telescope enclosure. In a normal situation, it takes about seven minutes to close (including slewing the telescope to stow position and moving the enclosure). However, our criteria for closing due to external conditions are somewhat conservative in case we must manually move the enclosure back over the telescope, which takes about 30 minutes. The observers will close the telescope during the night if any of the following conditions occur.

1. Any lightning is detected within 15 miles.

2. Precipitation is detected or a radar return from precipitation is approaching that is 30 minutes away or less.

3. Smoke or ash is detected.

4. Wind speed is over $40 \mathrm{mph}$.

5. Humidity is high, with a dewpoint $2.5 \mathrm{C}$ below ambient temperature. Observers also visually check for condensation any time the temperature drops within $4{ }^{\circ} \mathrm{C}$ of dewpoint.

6. Integrated dust counts (likely from the White Sands gypsum dunefield or agriculture in the typically dry valleys below the telescope) for the night exceeds 20,000-40,000 count hours. The exact number depends on humidity, closing at lower integrated dust hours during high humidity.

7. Ambient temperature is below $-12^{\circ} \mathrm{C}$.

At the end of each night, logs are distributed to a broad list of APO day staff, observers, software developers, and project leaders (including those for MARVELS, APOGEE, and SEGUE). These $\operatorname{logs}$ are partly generated automatically, covering most of the telescope, weather, and instrument status. Any problems or unusual situations are recorded manually by the observers, including relevant error messages and as much relevant information as is available.

\section{DATA QUALITY}

The final imaging observations of the BOSS footprint were completed in the Fall of 2009 and are found in DR8 (Aihara et al. 2011a). Following the spectrograph rebuild in Summer of 2009, BOSS commissioning took place in Fall 2009. During commissioning, spectroscopic observations were performed on nights assigned to BOSS when conditions were not photometric. Full spectroscopic survey operations began on 2009 December 5 (MJD 55170) after the commissioning phase was complete, marking the first spectroscopic data that are included in DR9. Several additional improvements were made to the spectrograph during the survey. A few bugs in the software for guiding were resolved, residual tilt in the CCD focal plane was corrected, new triplet lenses were installed for all four cameras, the red CCDs were replaced, and the collimator mirrors were recoated. These changes led to improved spectral resolution and throughput, with a combined improvement to survey efficiency of roughly 

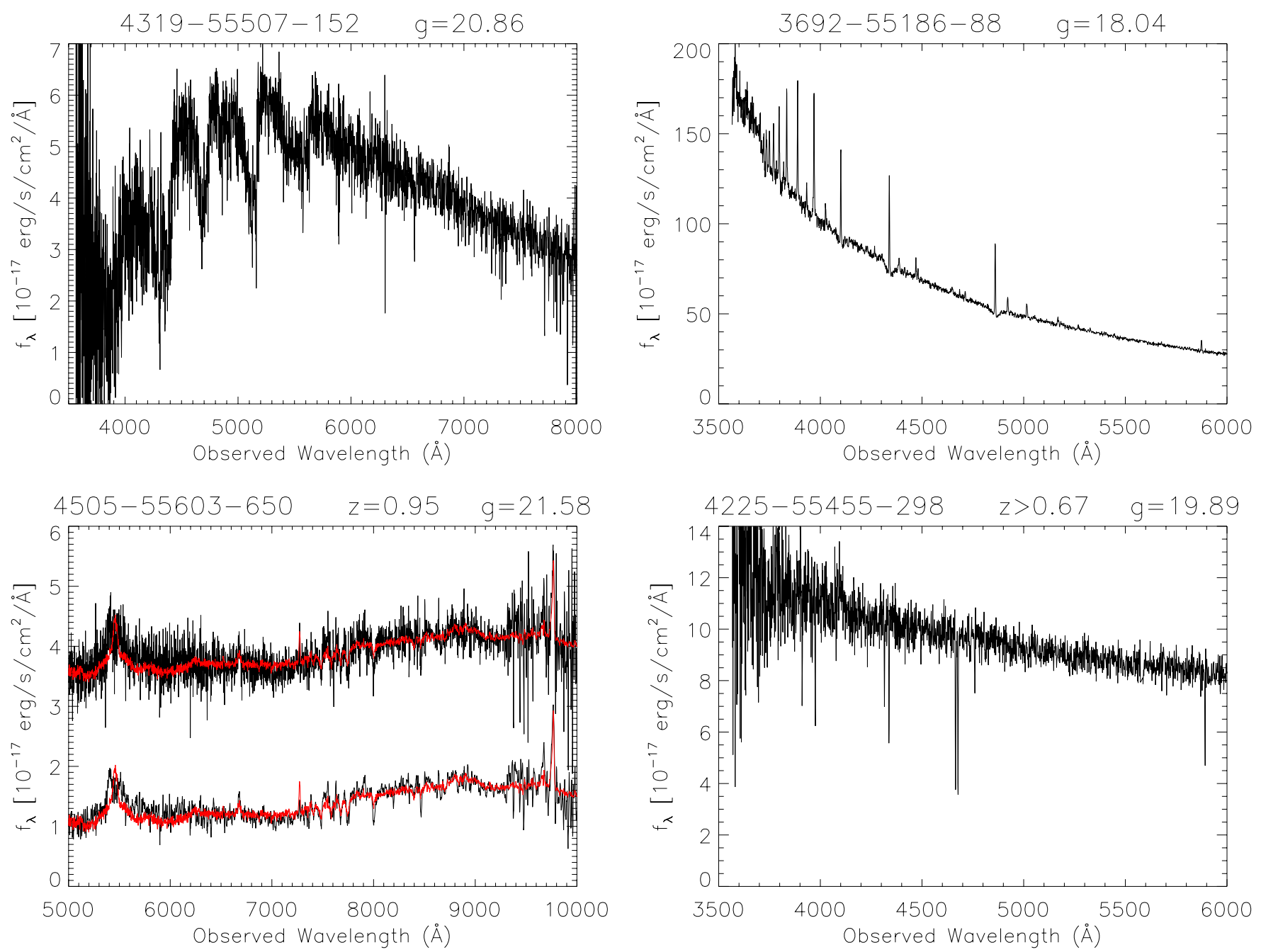

Figure 7. Mosaic of example spectra of rare objects discovered in the BOSS spectroscopic sample. Top: spectrum of a carbon star (left) and a cataclysmic variable with strong emission lines (right). Bottom: spectrum of a post-starburst galaxy at $z=0.95$ with AGN activity (left) and a featureless BL Lac object imprinted with absorption lines from Fe II $\lambda 2382$, Fe II $\lambda 2600$, and the Mg II $\lambda 2798,2803$ doublet at $z=0.67$ (right). The idlspec2d template (red) for the post-starburst galaxy is a good fit to the data. For this galaxy, the raw data are displayed with an offset of $2.5 \times 10^{-17} \mathrm{erg} \mathrm{s}^{-1} \mathrm{~cm}^{-2} \AA^{-1}$ and the spectrum smoothed with a five-pixel median boxcar filter is shown at true flux density.

(A color version of this figure is available in the online journal.)

25\%. The dates of these changes are documented in Ahn et al. (2012) and D. Schlegel et al. (2013, in preparation).

To give the reader a qualitative impression of the BOSS data quality from these first two years of data, we present a few examples of galaxy, quasar, and stellar spectra in Figure 6. As with SDSS, the enormous size of the BOSS spectroscopic sample also includes many classes of rare objects. A few examples of unusual spectra are shown in Figure 7. In the following section, we describe some global characteristics of the BOSS spectra.

\subsection{BOSS Spectrophotometry}

As described briefly in Section 4.3, the flux calibration for each exposure is corrected as a function of position in the focal plane using PSF magnitudes of standard stars as a baseline. Centroiding errors, extended objects, guiding errors, transparency variations, and other uncertainties in the corrections can lead to spectrophotometric errors above the measurement uncertainty over the $3^{\circ}$ field. As explained in Tremonti et al. (2004), an analysis of magnitudes synthesized from SDSS galaxy spectra showed an rms dispersion of 5\% in $(g-r)$ and 3\% in $(r-i)$ relative to the colors measured from $3^{\prime \prime}$ fiber magnitudes from SDSS photometry. At the bluest wavelengths ( $3800 \AA$ ) , the error was closer to $12 \%$. Comparing stars with a PSF magnitude brighter than 19, the SDSS spectrophotometry was biased 0.02 mag brighter with an rms dispersion of 0.05 mag in $r$ (DR6; Adelman-McCarthy et al. 2008). Similarly, the $(g-r)$ colors showed a bias of 0.02 mag with 0.05 mag dispersion while the $(r-i)$ colors showed a bias of $-0.01 \mathrm{mag}$ with $0.03 \mathrm{mag}$ dispersion.

We performed a similar analysis on the flux calibration of BOSS spectra using objects with $15<g_{\text {fib } 2}<19$. As shown in Figure 8, we find slightly larger bias and rms dispersion in fluxing errors for stars and galaxies than was reported in SDSS. The larger fluxing errors are not surprising because the BOSS fibers are smaller in diameter and therefore more susceptible to guiding offsets. For the standard stars we find BOSS spectrophotometry to be on average $0.014 \mathrm{mag}$ fainter than the PSF photometry with an rms dispersion of $0.058 \mathrm{mag}$ in $r$. BOSS spectrophotometry is $0.038 \mathrm{mag}$ fainter (0.068 mag dispersion) than the PSF photometry in $g$. The $(g-r)$ colors are 0.022 mag redder $(0.063$ mag dispersion) while 

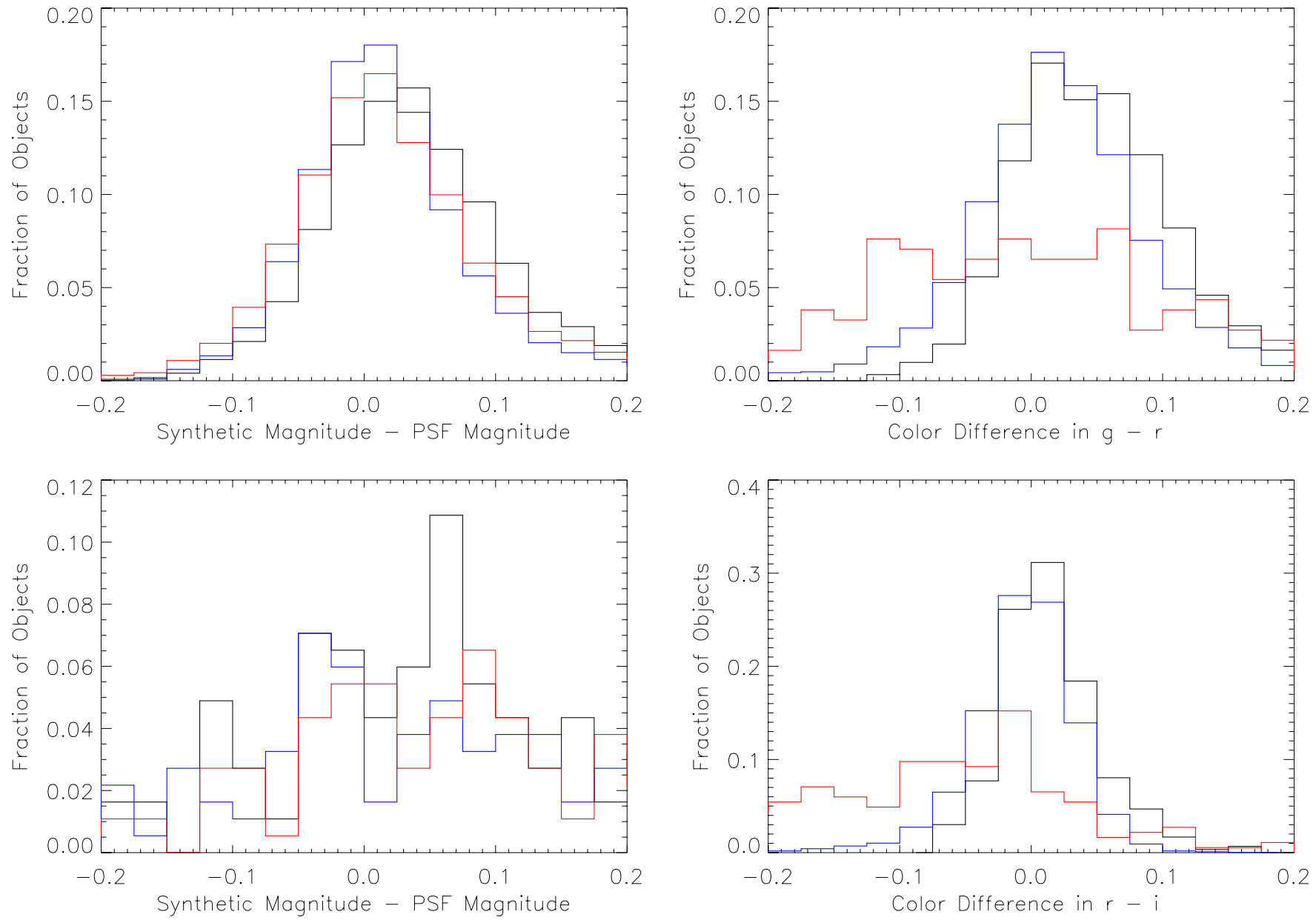

Figure 8. Difference between the synthetic photometry computed from each spectrum and the measured photometry from the SDSS imaging data. Left: histogram of the spectrophotometric offsets for stars. The black line shows $g_{\text {synthetic }}-g_{\text {PSF }}$ for the standard stars (top) and for a sample of stars that appeared as contaminants in the CORE and BONUS quasar samples (bottom). Similarly, the blue lines show the offsets for the $r$ filter while the red lines show the offsets for the $i$ filter. Right: histogram of the color differences $(g-r)_{\text {synthetic }}-(g-r)_{\text {PSF }}$ shown in the top panel and $r-i$ in the bottom panel. Standard stars are presented as the blue line using PSF magnitudes, galaxies as the black line (fiber2 magnitudes), and stellar contaminants in the quasar sample as the red line (PSF magnitudes).

(A color version of this figure is available in the online journal.)
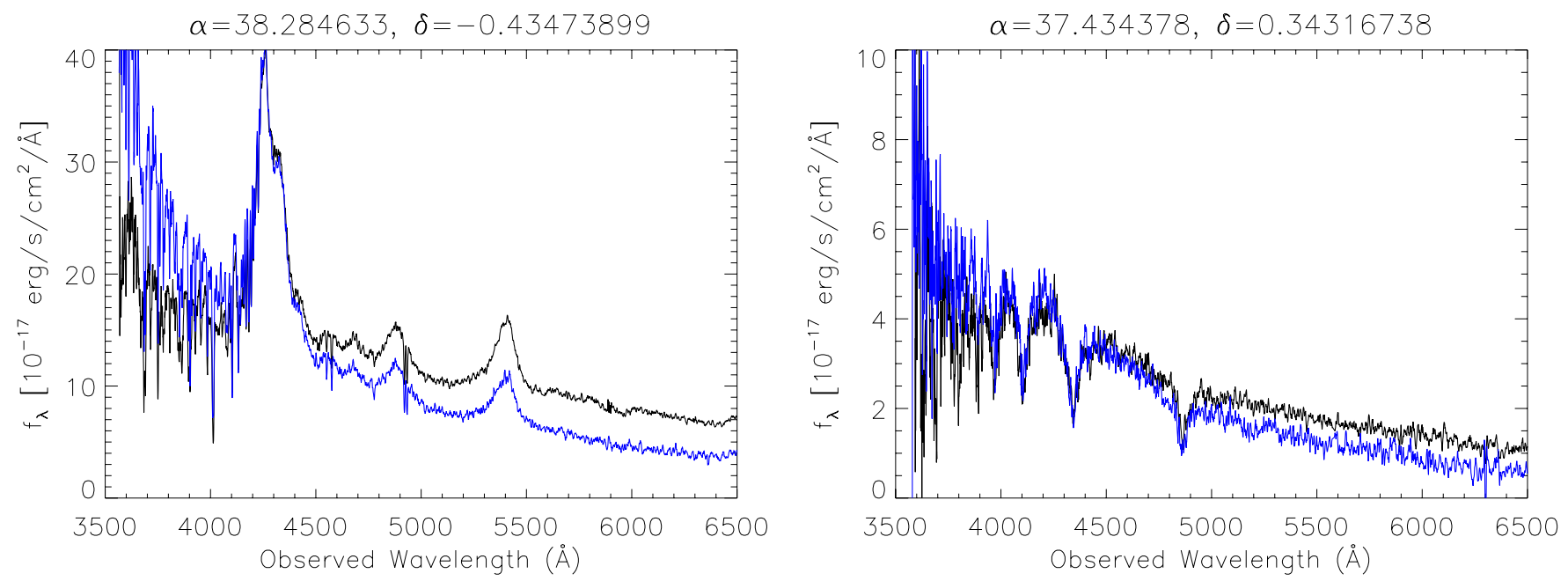

Figure 9. Quasar target spectra smoothed with a five-pixel median boxcar filter, covering the wavelength range $3500 \AA \AA$ through $6500 \AA$ taken at different airmass, demonstrating the effects of atmospheric differential refraction and other guiding errors on spectrophotometry. Left: spectrum of a $g_{\text {fib } 2}=19.48$ quasar at $z=2.49$ observed at an airmass of 1.2 (black) and again at an airmass of 1.4 (blue). Right: spectrum of a white dwarf with $g_{\text {fib } 2}=21.53$ on the same observations with the same color pattern.

(A color version of this figure is available in the online journal.) 
the $(r-i)$ colors are 0.004 mag bluer ( 0.035 mag dispersion). For galaxies, we compare only colors, using the $2^{\prime \prime}$ fiber magnitudes from SDSS imaging, and find that $(g-r)$ colors are 0.048 ( $0.058 \mathrm{mag}$ dispersion) and $(r-i)$ colors are $0.013 \mathrm{mag}$ (0.035 mag dispersion) redder for the spectra. As shown in the same figure, the offsets and dispersion of spectra are much larger for objects targeted as quasars but confirmed to be stars. On average, stellar contaminants in the quasar sample are $0.16 \mathrm{mag}$ fainter in $r$, with an rms dispersion of $0.29 \mathrm{mag}$; these objects are 0.038 (0.158 mag dispersion) and 0.070 (0.099 mag dispersion) magnitudes more blue in $g-r$ and $r-i$ colors, respectively.

The application of washers and the offsets in plate position for the quasar fibers accounts for the warping of the quasar target spectrophotometry relative to the standard stars. The quasars have higher throughput in the blue relative to the standard stars at a cost of reduced throughput in the red, and this difference is not included in the flux calibration. The effect is exacerbated by the smaller fibers (now comparable in diameter to the typical $1^{\prime \prime} .5$ seeing at Apache Point) relative to those used in SDSS.

The net effects of these sources of error can be demonstrated in the spectrophotometry of plates 3615 and 3647 , both located on the celestial equator at $\alpha=37^{\circ}$. The two plates contain identical targets but were drilled for different airmass and were observed a total of nine times in the first two years to monitor the system. An example of the spectrophotometric calibration accuracy from these two plates is shown in Figure 9. The left panel displays a quasar observed twice at different airmass. Note the clear trend toward bluer synthetic photometry in the spectrum that was obtained at larger airmass; ADR causes the red light to be offset from the center of the fiber for the quasar target in an opposite sense to the ADR effect on the spectrophotometric star fibers (optimized for throughput at $5400 \AA$ ). Also shown in the figure is the spectrum of a star that was targeted as a likely quasar and therefore positioned in the offset quasar focal plane. The stellar profile is known to be constant over the period of 29 days between the two observations, yet the spectra show the same trend toward bluer colors.

An understanding of spectrophotometric accuracy is critical for studies of cosmology, galaxy evolution, and quasar physics. Yan (2011) evaluates small wavelength-scale residuals in the flux calibration that can contaminate weak emission and absorption features in the spectra. Using SDSS spectra, he estimates that the wavelength-dependent relative flux calibration is accurate at the 1\%-2\% level. A similar analysis has not been published with BOSS spectra, but the similarities in the data reduction pipeline make it likely that the BOSS spectrophotometry has similar accuracy on small wavelength scales. The broadband spectrophotometry accuracy described here is biased at less than 5\% for the standard stars and galaxy targets, with a comparable amount of scatter. This precision does not seem to significantly reduce the galaxy redshift efficiency; as was shown in Section 5.1, the $i_{\text {fib2 }}<21.5$ CMASS spectra are successfully classified for more than $94 \%$ of the objects. The estimates of color scatter and bias described in this section should be taken into consideration for science applications that require precise spectrophotometry; repeat spectra can be used to verify the reproducibility of measurements. In general, galaxy evolution studies should use the $2^{\prime \prime}$ fiber magnitudes for comparison of spectrophotometric color to photometry, as the central region of extended galaxies contributes the majority of light to the BOSS spectra. The intrinsic quasar continuum must be estimated before Ly $\alpha$ forest analysis and is highly susceptible to spectrophotometric errors because of the fiber offsets. The Ly $\alpha$ analysis of Slosar et al. (2011) effectively treats the broadband power introduced by spectrophotometric errors as a nuisance parameter in continuum fitting. The continuum fitting procedure outlined in Lee et al. (2012) has been applied to the BOSS data and will be described in a public release of model quasar continuum (Lee et al. 2012).

\subsection{Galaxy Spectroscopic Data Quality}

Thomas et al. (2012) perform a spectroscopic analysis of the 492,450 galaxy spectra that are part of DR9. They show that the typical signal-to-noise ratio of BOSS spectra is sufficient to measure simple dynamical quantities such as stellar velocity dispersion for individual objects. The typical error in the velocity dispersion measurement is $14 \%$, and $93 \%$ of BOSS galaxies have velocity dispersions with an accuracy better than $30 \%$. Thomas et al. (2012) further show that emission lines can be measured on BOSS spectra, but the majority of BOSS galaxies lack detectable emission lines, as is to be expected because of the target selection design toward massive galaxies.

In order to independently assess the performance of BOSS idlspec $2 d$ in determining galaxy redshifts, the code "RUNZ" 81 was run on all extracted galaxy spectra. Comparisons between the RUNZ and idlspec $2 \mathrm{~d}$ redshifts gave important indications of common failure modes in the idlspec $2 \mathrm{~d}$ redshifts at the beginning of the survey. The results from RUNZ will be made available with DR9.

RUNZ was originally developed for 2dFGRS (Colless et al. 2001) and has since been optimized for several LRG redshift surveys such as the 2SLAQ Survey (Cannon et al. 2006) and the AAOmega UKIDSS SDSS LRG Survey (D. A. Wake et al. 2013, in preparation). The software has also been used to determine redshifts for emission line galaxies in the WiggleZ survey (Drinkwater et al. 2010) and for a broad range of galaxy types in the Galaxy and Mass Assembly survey (Driver et al. 2009, 2011). RUNZ provides two redshift estimates for each input spectrum; the first by cross-correlating each spectrum with a series of galaxy and stellar templates (with the emission lines masked) and the second by fitting a Gaussian to detected emission lines and identifying multiple matches at a single redshift. An integer value $(q)$ between one and five is assigned to reflect the quality of the redshift. RUNZ was modified for BOSS and tuned to assign quality values and input templates based on the visual inspection of one of the commissioning plates.

Selecting all repeat spectra, we identify the primary spectrum (also requiring $q>3$ ) and test the reliability of the RUNZ quality flags and redshifts assigned to the other spectra of the same object. We attempted to tune the quality value $q=3$ to correspond to a $95 \%$ reliability and $q=4$ to correspond to a $>99 \%$ reliability. Table 5 shows the fraction of repeat spectra pairs that yield the same redshifts, where agreement is defined as a velocity difference $\delta v=(z 1-z 2) /(1+z 2) * c<500 \mathrm{~km} \mathrm{~s}^{-1}$. Analogous statistics are reported in Table 5 for the idlspec $2 \mathrm{~d}$ redshifts, where a reliable redshift is designated as having ZWARNING $=0$. The results agree with the findings of Ross et al. (2012a), who report that LOWZ and CMASS galaxies that were observed multiple times produced idlspec $2 \mathrm{~d}$ redshifts with a deviation $\delta z /(1+z)<0.001$ in $99.7 \%$ of the cases when the spectroscopic classification produced ZWARNING $=0$. Visual inspection of a subsample of those data confirmed this result, indicating that the rate of catastrophic failures among

\footnotetext{
81 Maintained by Scott Croom.
} 
Table 5

Redshift Consistency of Repeat Spectra

\begin{tabular}{lccc}
\hline \hline $\begin{array}{l}\text { Condition } \\
\text { of Object } 1\end{array}$ & $\begin{array}{c}\text { Condition } \\
\text { of Object 2 }\end{array}$ & $\begin{array}{c}\text { Number } \\
\text { of Pairs }\end{array}$ & $\begin{array}{c}\text { Consistency } \\
\text { Fraction }\end{array}$ \\
\hline$q \leqslant 2$ & $q>3$ & 448 & 0.58 \\
$q=3$ & $q>3$ & 1298 & 0.93 \\
$q>3$ & $q>3$ & 24075 & 0.996 \\
$q \geqslant 3$ & $q>3$ & 25373 & 0.992 \\
ZWARN $=0$ & ZWARN $=0$ & 27304 & 0.996 \\
ZWARN $>0$ & ZWARN $=0$ & 771 & 0.68 \\
\hline
\end{tabular}

the galaxy targets is less than $1 \%$, as required to meet the BAO requirements.

We estimated the redshift accuracy for RUNZ and for idlspec $2 \mathrm{~d}$ by using the same repeat spectra and requiring all pairs to have $q>2$ (or ZWARNING $=0$ ). The distribution of $\delta v$ for RUNZ and BOSS are shown in the bottom panel of Figure 10. The idlspec $2 \mathrm{~d}$ redshifts are significantly more repeatable, indicating a single-epoch rms uncertainty of $38 \mathrm{~km} \mathrm{~s}^{-1}$ compared with $81 \mathrm{~km} \mathrm{~s}^{-1}$ for the RUNZ redshifts (after removing pairs with $\delta v>2000 \mathrm{~km} \mathrm{~s}^{-1}$ ). The bottom panel of Figure 10 shows

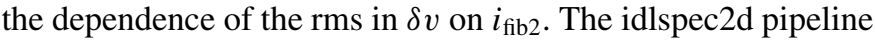
now performs better at all magnitudes, continues to improve at bright magnitudes, and grows more slowly with decreasing flux when compared with the RUNZ results.

When comparing the results of RUNZ directly to the idlspec $2 \mathrm{~d}$ pipeline for identical spectra, we find the RUNZ redshifts are lower by a median of $8 \mathrm{~km} \mathrm{~s}^{-1}$. The rms scatter between the two algorithms is $72 \mathrm{~km} \mathrm{~s}^{-1}$, consistent with the larger rms scatter observed in the repeat spectra of RUNZ redshifts. Possible redshift failure modes are identified by comparing instances where both idlspec $2 \mathrm{~d}$ and RUNZ indicate a correct redshift for a galaxy but the redshifts differ significantly. There are 311 such galaxies in DR9 where ZWARNING $=0, q>3$ and $\left(z_{\text {RUNZ }}-z_{\text {idlspec } 2 \mathrm{~d}}\right) /\left(1+z_{\text {idlspec } 2 \mathrm{~d}}\right) * c>1000 \mathrm{~km} \mathrm{~s}^{-1}$. Visual inspection reveals a number of causes for the failures. In many cases, both redshifts are valid because a superposition of two different objects at different redshifts leads to two correct results; two such examples are presented in Figure 11. In the case of a star-galaxy superposition, the idlspec2d pipeline mildly favors a redshift assigned to the stars compared to RUNZ. However, in the case that background emission-line objects are projected on foreground galaxy light, idlspec $2 \mathrm{~d}$ tends to select the background emission-line redshift whereas RUNZ selects the foreground redshift. A genuine failure mode for idlspec $2 \mathrm{~d}$ appears in instances of bad data, either where the relative normalization of the red and blue spectra has failed or some broadband shape has been introduced into the blue spectra. An example of such a spectrum is shown in the left hand panel of Figure 12. RUNZ appears to be less affected by such problems, as it doesn't fit the broadband shape of the spectra. The most common failure for RUNZ occurs in strong emission-line galaxies. These failures may result from inadequate templates combined with an insufficient weight given to the emission-line redshift relative to the cross-correlation redshift as a result of the LRG optimization of this version of RUNZ.

While RUNZ was used to help diagnose BOSS galaxy redshifts early in the survey, we concentrated our subsequent software development entirely on the idlspec $2 \mathrm{~d}$ data reduction pipeline and redshift classification. The algorithms employed for redshift determination of BOSS targets are described in Bolton et al. (2012b), including a more thorough description of
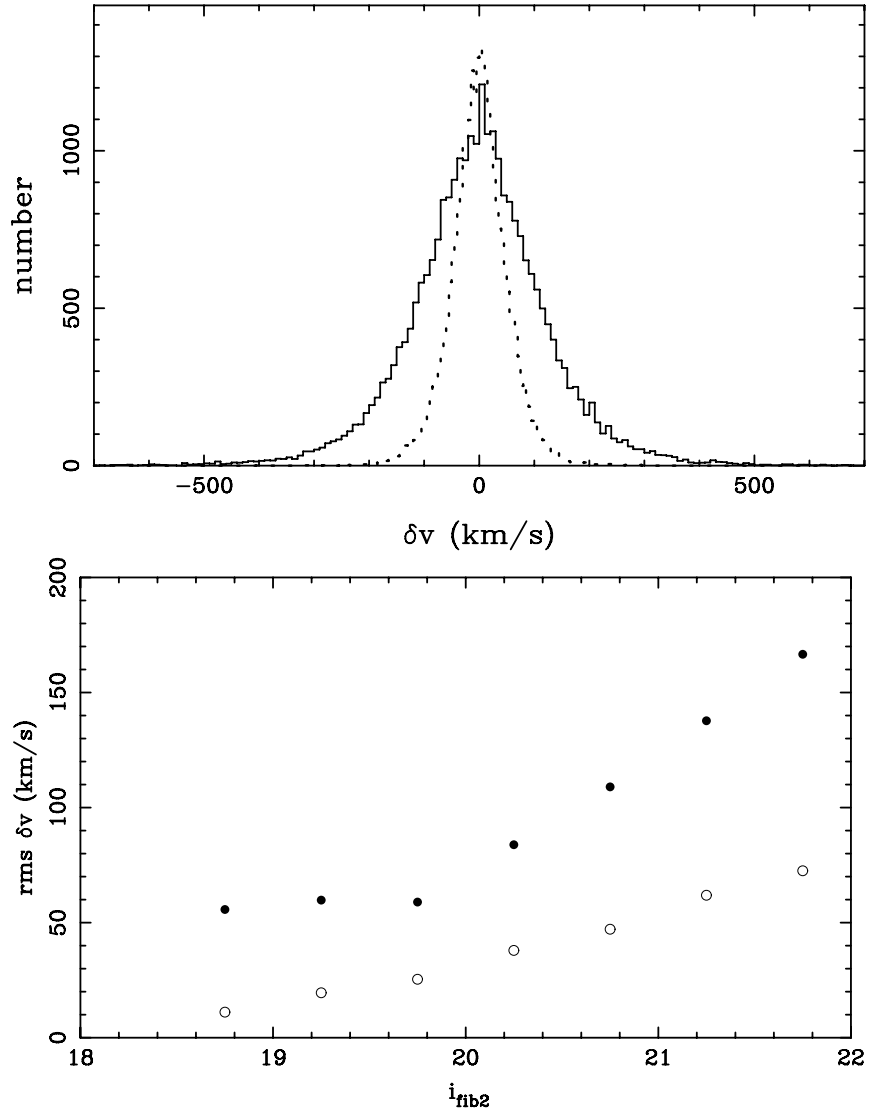

Figure 10. Statistics of $\delta v$ in pairs of spectra for the same galaxy. Top: histogram of $\delta v$ for RUNZ (solid black) and for idlspec2d (dashed). Bottom: rms width of distribution binned at half magnitude intervals in $i_{\text {fib2 }}$. RUNZ is presented as solid circles while idlspec $2 \mathrm{~d}$ is presented as open circles.

templates and redshift efficiency than what is found here. Even though idlspec $2 \mathrm{~d}$ performs slightly better than RUNZ in terms of completeness, reliability, and redshift repeatability, RUNZ would probably perform better if it were fully optimized for the BOSS data.

\subsection{Quasar Classification}

Due to the variety of quasar spectral features and the complexity of automatic classification, members of the French Participation Group supplemented the idlspec $2 d$ results with a visual inspection of the spectra. The visual inspection provides a secure identification and a reliable estimate of the redshift for each object in addition to a characterization of other fundamental quasar properties. In total, 189,018 spectra were inspected in the DR9 sample. We provide a brief summary of the inspections; full details are found in Pâris et al. (2012).

All objects that were targeted as quasars or classified as quasars with $z \geqslant 2$ by idlspec2d were visually inspected and added to the DR9 quasar catalog (DR9Q; Pâris et al. 2012), which is included with DR9. The inspections include not only CORE and BONUS quasars, but also objects from ancillary programs focused on quasars. A fraction of objects in the galaxy sample were identified as quasars by idlspec $2 \mathrm{~d}$ and visually inspected as well. The catalog provides human classifications of the objects, refined redshift estimates and emission line characteristics. Peculiar spectral features that could affect any $\operatorname{Ly} \alpha$ forest analysis, such as damped Ly $\alpha$ (DLA) systems and broad absorption line (BAL) quasars, are first flagged in 

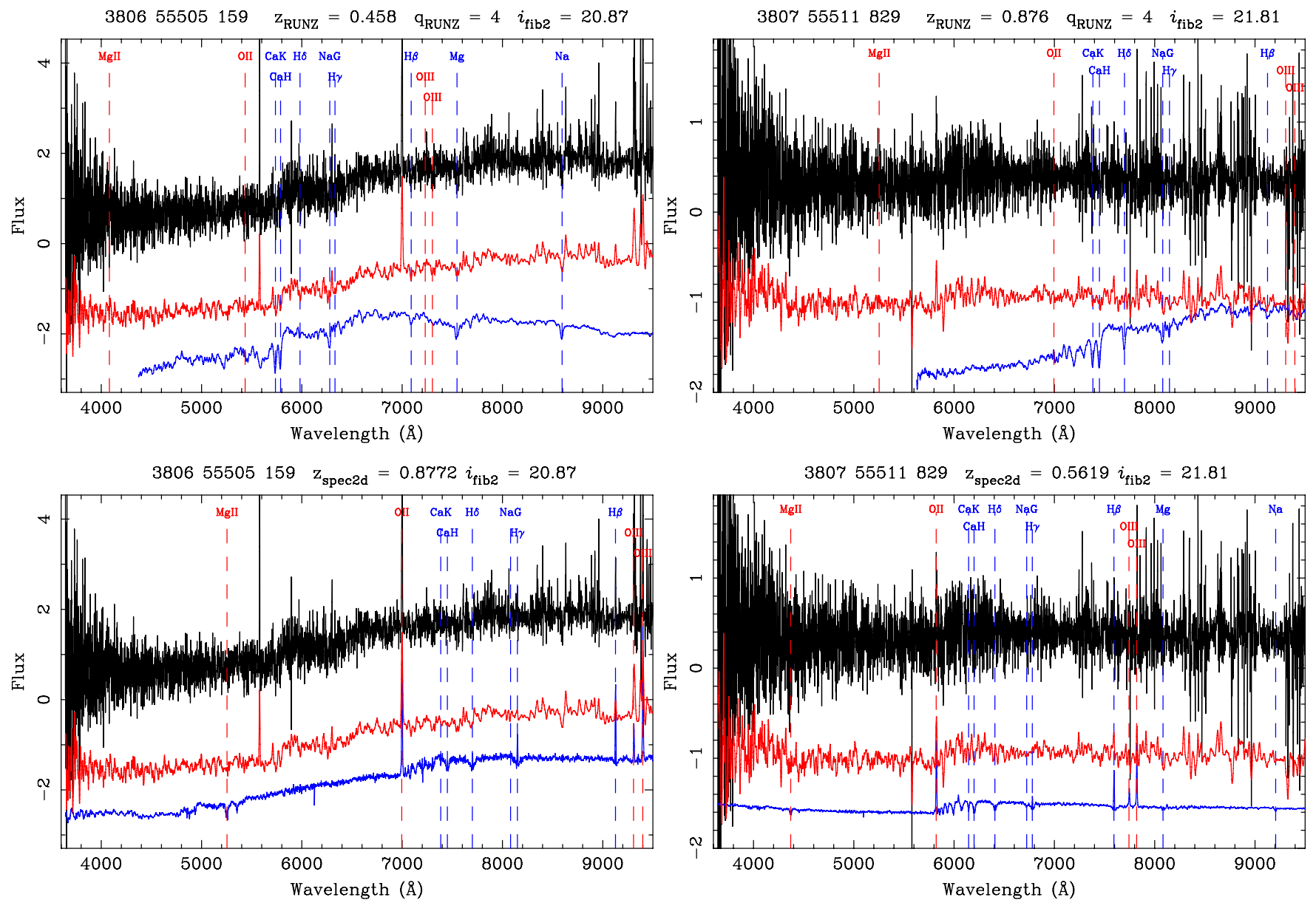

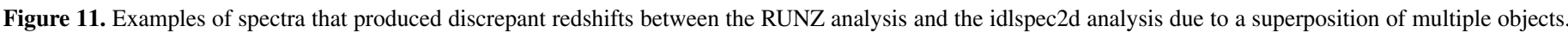

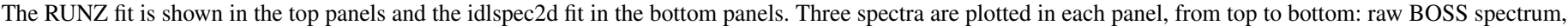

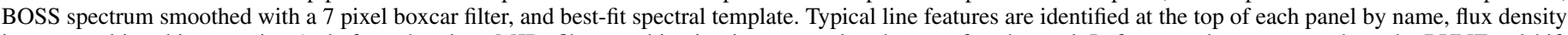

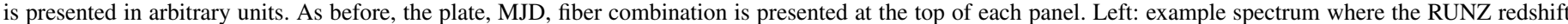

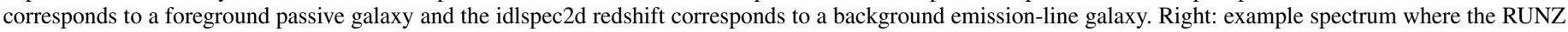
redshift corresponds to a background passive galaxy and the idlspec $2 \mathrm{~d}$ redshift corresponds to a foreground emission-line galaxy.

(A color version of this figure is available in the online journal.)

visual inspection. DLA systems are then evaluated with an automated characterization to determine column densities (Noterdaeme et al. 2009). Those results will be reported in a separate catalog (Noterdaeme et al. 2012). BAL quasars are processed for automatic estimates of the balnicity index (BI; Weymann et al. 1991) and absorption index (AI; Hall et al. 2002) of C IV troughs to quantify their strength. Problems identified in visual inspection such as the presence of artificial breaks in the spectrum, poor flux calibration, or bad sky subtraction are flagged as well.

The visual inspection starts from the output of idlspec $2 \mathrm{~d}$ for both classification and redshift. We find that fewer than $0.3 \%$ of objects classified as quasars with ZWARNING $=0$ by idlspec $2 \mathrm{~d}$ have misidentified the observed emission lines (typically at $z<2$, where $\operatorname{Ly} \alpha$ emission is not observable), leading to redshift errors $\Delta z>0.1$. About half of the objects classified as quasars by idlspec2d have redshifts adjusted by less than $\Delta z>0.1(\overline{\Delta z}<0.005)$ after their visual inspection, when the best fit idlspec2d template misses the position of the $\mathrm{Mg}$ II emission line peak or when the maximum of the C IV emission line determines the redshift. The precision of quasar redshifts are further refined using a linear combination of carefully crafted principal components fit to each spectrum (see, e.g., Pâris et al. 2011). Indeed, known shifts between emission lines are intrinsically imprinted in the eigenvectors and this method also takes into account the quasar-to-quasar variation. This redshift estimate is also provided in addition to the idlspec $2 \mathrm{~d}$ results as part of the DR9Q.

About $12 \%$ of the quasar targets in the CORE and BONUS samples are assigned a non-zero ZWARNING flag in the idlspec $2 d$ pipeline. Visual inspection reveals that $13 \%$ of these objects are truly quasars and about $7 \%$ have $z>2.15$. Of those objects that are classified with ZWARNING $=0$ as quasars in idlspec2d, approximately $1 \%$ are revealed to be stars. Similarly, only 23 objects classified as stars with ZWARNING $=0$ are shown to be quasars in visual inspection, only five of which are $z>2.15$ quasars. A summary of the classifications in DR9Q is provided in Table 6.

\subsection{Ly $\alpha$ Forest Measurements}

As described in Section 5.2, the exposure depths over the full survey are sufficient to ensure a nearly $100 \%$ redshift completeness among true quasars. McQuinn \& White (2011) also address the question of a survey's sensitivity in the Ly $\alpha$ forest using a metric $v_{n}$. The parameter $v_{n}$ ranges from 0 to 1 and represents the effective value of each quasar to constraining 

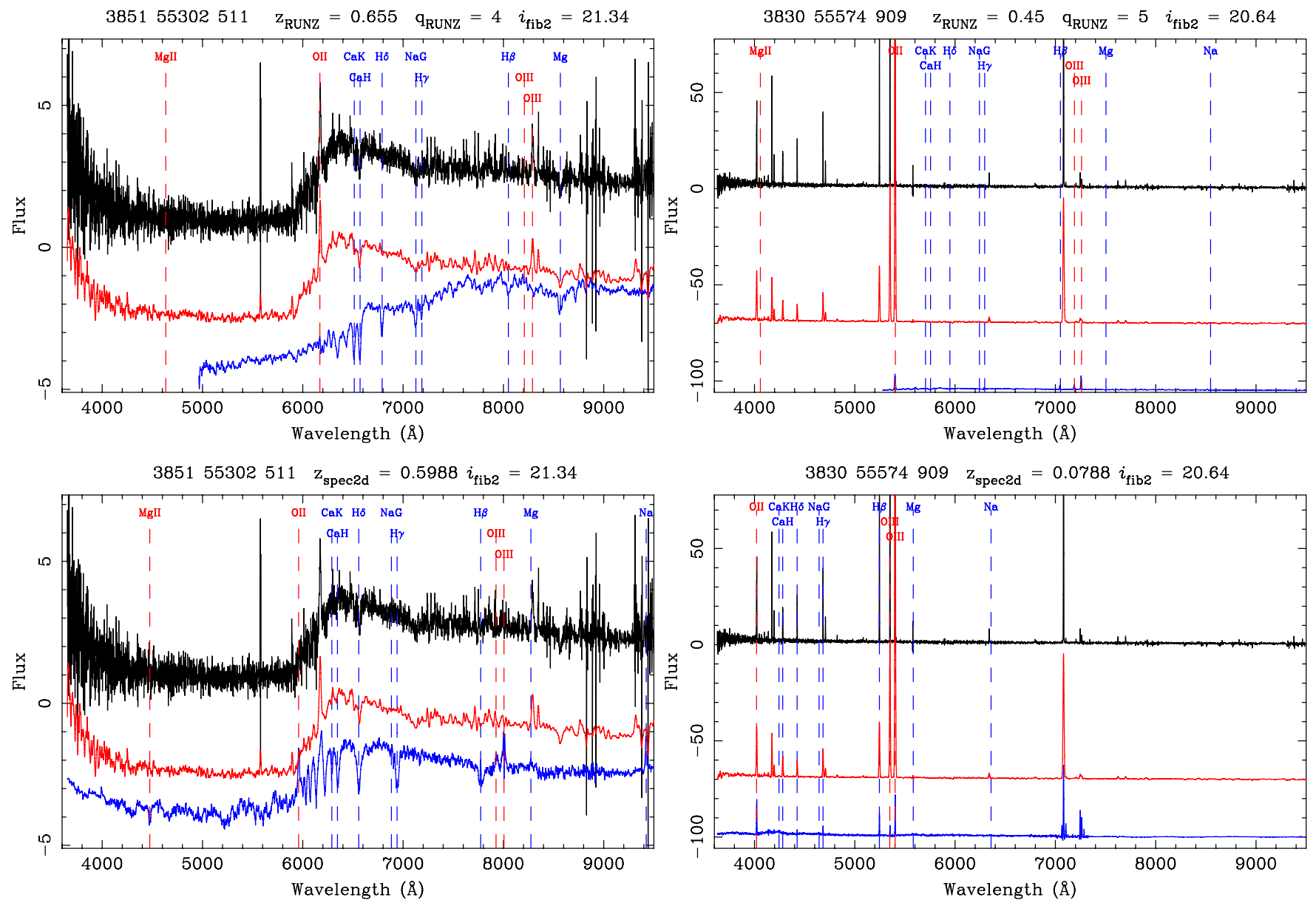

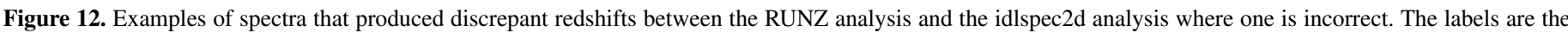

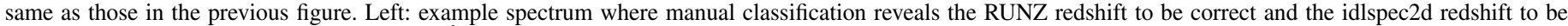

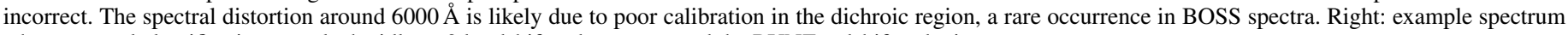
where manual classification reveals the idlspec $2 \mathrm{~d}$ redshift to be correct and the RUNZ redshift to be incorrect.

(A color version of this figure is available in the online journal.)

Table 6

Key Statistics from DR9Q

\begin{tabular}{lcc}
\hline \hline Classification & No. of Visually Inspected & Fraction \\
\hline Quasar & 87,822 & 1.00 \\
Quasar with $z \geqslant 2.15$ & 61,933 & 0.70 \\
BAL quasar & 7,532 & 0.086 \\
DLA quasar & 7,492 & 0.085 \\
\hline
\end{tabular}

the three-dimensional power spectrum depending on the redshift and $\mathrm{S} / \mathrm{N}$ in the Ly $\alpha$ forest region. Assuming the quasar redshift and luminosity distribution of Hopkins et al. (2006), they find that $v_{n}$ roughly doubles as $\mathrm{S} / \mathrm{N}$ per $\AA$ increases from one to two for a quasar at $z \sim 3$. Similar to Font-Ribera et al. (2012), they argue for a survey that favors area over depth, citing specifically that the improvement in sensitivity is marginal once the noise exceeds $\mathrm{S} / \mathrm{N}=2$ per $\AA$ for the faintest quasars. The specific Ly $\alpha$ data quality modeled in these projections lends themselves to a direct comparison to the BOSS data obtained in the first two years of the survey.

Using the DR9 quasar sample, we evaluate the depth of the spectra in the Ly $\alpha$ forest for comparison to the McQuinn \& White (2011) projections. Using only objects that were classified as quasars by idlspec $2 \mathrm{~d}$ with ZWARNING $=0$, we compute the $\mathrm{S} / \mathrm{N}$ per co-added BOSS pixel between $1041 \AA$ and $1185 \AA$ in the rest frame of each quasar. In the co-added spectra, the sampling at $4300 \AA$ corresponds to roughly $1 \AA$ A per pixel, leading to a sampling that is easily compared to the projections of McQuinn \& White (2011). The sampling of the Ly $\alpha$ forest is somewhat higher for quasars at $z=2.15$ and slightly lower for quasars at $z=3.5$. The $\mathrm{S} / \mathrm{N}$ statistics of the Ly $\alpha$ forest pixels are shown in Figure 13. The typical quasar with $g_{\mathrm{PSF}}<21.5$ exceeds $\mathrm{S} / \mathrm{N}=2$ per pixel while the fainter quasars exceed $\mathrm{S} / \mathrm{N}=1$ on average, leading to values $v_{n}=0.5$ and $v_{n}=0.2$ at $z=2.6$ for the two samples, respectively. The target density and survey depth results in an $\mathrm{S} / \mathrm{N}$ that is sufficient for Ly $\alpha$ constraints on the BAO peak.

\section{CONCLUSION}

The data from the 2009-2010 and the 2010-2011 BOSS observing seasons are included in DR9. Observations followed a pace that was close to the survey projections; 236 unique plates were completed in first year compared with an expectation of 331. While this rate was about $30 \%$ behind the required rate to complete the survey, the slow start can largely be explained by the deeper exposures that were acquired. The $\mathrm{S} / \mathrm{N}^{2}$ thresholds were reduced by $37.5 \%$ on the blue cameras and $15 \%$ on the red cameras after that first year; 577 unique plates were completed in 

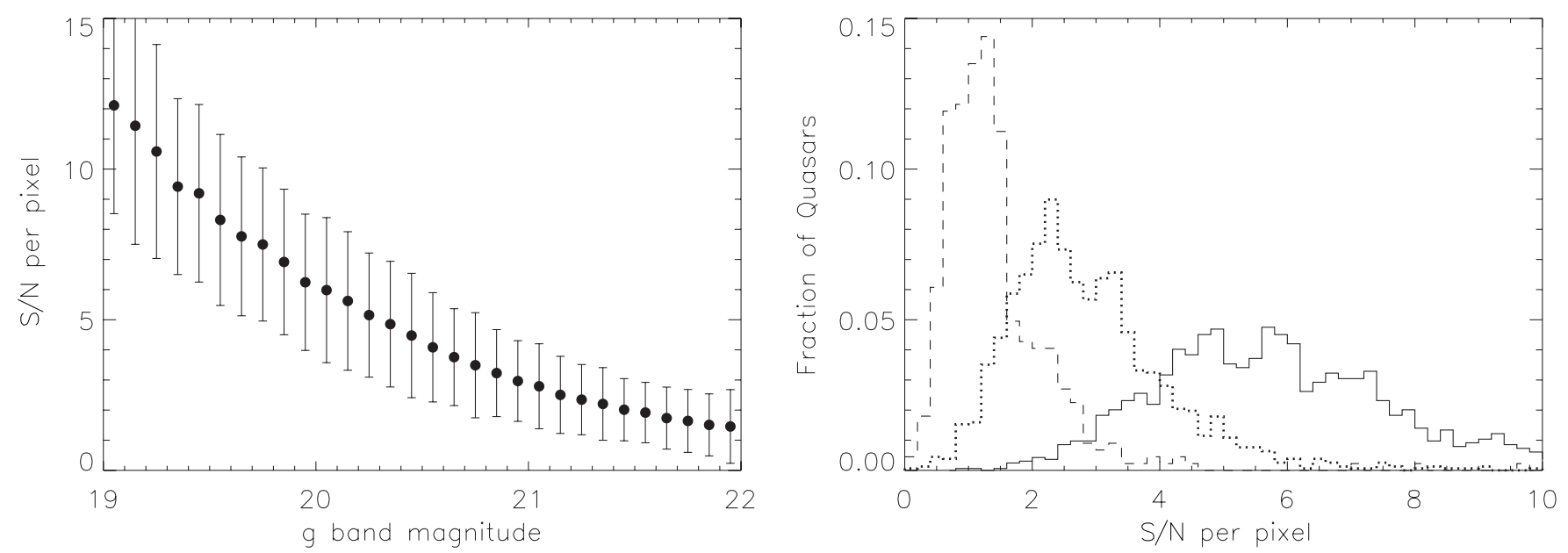

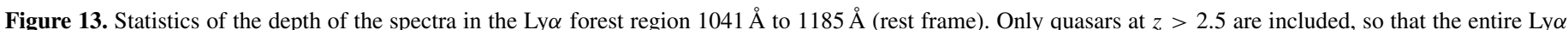

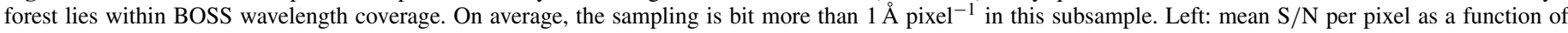

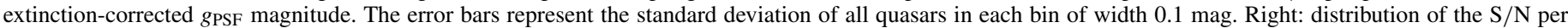

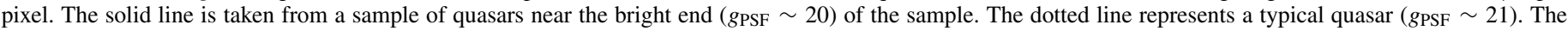
dashed line shows quasars near the faint magnitude limit $g_{\mathrm{PSF}} \sim 22.0$ of the CORE and BONUS target selection algorithms.
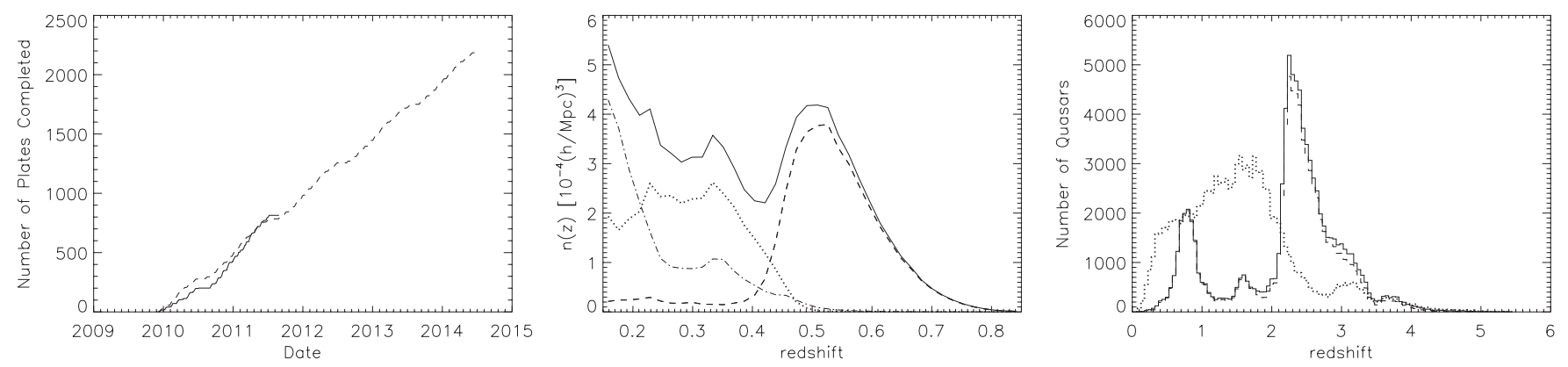

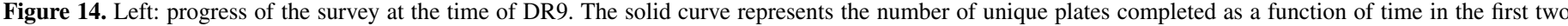

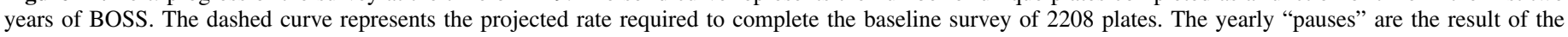

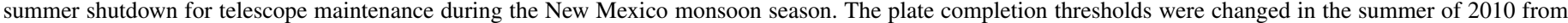

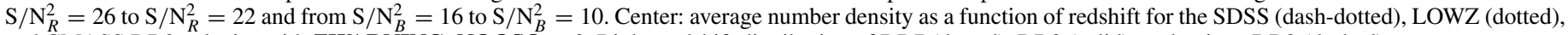
and CMASS DR9 galaxies with ZWARNING_NOQSO = 0. Right: redshift distribution of DR7 (dotted), DR9 (solid), and unique DR9 (dashed) quasars.

the second year, compared with an expectation of 454 . The rate of plate completion benefited from better than average weather in the spring of 2011. In total, 813 unique plates have been completed, $3.5 \%$ ahead of the projected progress to complete the survey by the summer of 2014. As reported in Anderson et al. (2012), these plates account for $3275 \mathrm{deg}^{2}$ of unique sky coverage, approximately $35 \%$ of the full footprint. The rate of plate completion as a function of time during the first two years of BOSS is shown in Figure 14.

The plates completed as a function of equatorial coordinates are presented in Figure 1. This figure reveals a high rate of completion in the tGC and a lower rate in the SGC. The difference between the NGC and SGC is due to the unusually good weather in the Spring of 2011 and the fact that commissioning occurred during most of the time that the SGC was visible in 2009. The difference is more acute when comparing the $110^{\circ}<\alpha<130^{\circ}$ region of the $\mathrm{NGC}$ to the $\alpha>330^{\circ}$ region of the SGC. As explained in Section 5.4, the Galactic plane crosses at LST $\sim 4.6 \mathrm{hr}$, and many of those NGC fields must be observed near that time. As shown in Figure 5, the shorter nights and New Mexico monsoon season result in a smaller amount of observing time available to observe those SGC plates. Looking beyond the second year and assuming average weather, we predict that we will complete the NGC region of the survey in less than the allotted time, but will likely need better than average weather to finish the $\alpha>330^{\circ}$ region of the SGC.

The DR9 sample is the first sample of BOSS spectra released to the public and is the data that define the initial sample for the BOSS galaxy and Ly $\alpha$ BAO analyses described below. DR9 contains 324,198 unique CMASS targets and 29,493 repeat observations from the CMASS sample. Exceeding survey requirements, the spectra from $98.7 \%$ of the CMASS targets produced ZWARNING_NOQSO $=0$ in at least one of the observations, and $95 . \overline{4} \%$ were confirmed as galaxies. Contamination from stars accounts for the $3.3 \%$ of successfully classified objects that were not galaxies. As expected, the statistics from the LOWZ sample are even better; 103,729 unique targets $(7,646$ repeat observations) produced a $99.9 \%$ successful rate of classification; $99.2 \%$ of LOWZ targets were successfully classified as galaxies.

There were 154,433 unique quasar targets from the main sample, with 11,601 repeat observations. The idlspec $2 \mathrm{~d}$ pipeline classified $79.2 \%$ of these objects successfully and determined $51.5 \%$ of the 154,433 objects to be quasars. The numbers are similar to the numbers found in manual classification described in Section 6.3, with minor differences explained by the inclusion of ancillary programs in the manual inspections and occasional disagreement between the two techniques. A summary of the DR9 statistics is found in Table 7 and the redshift distribution for 

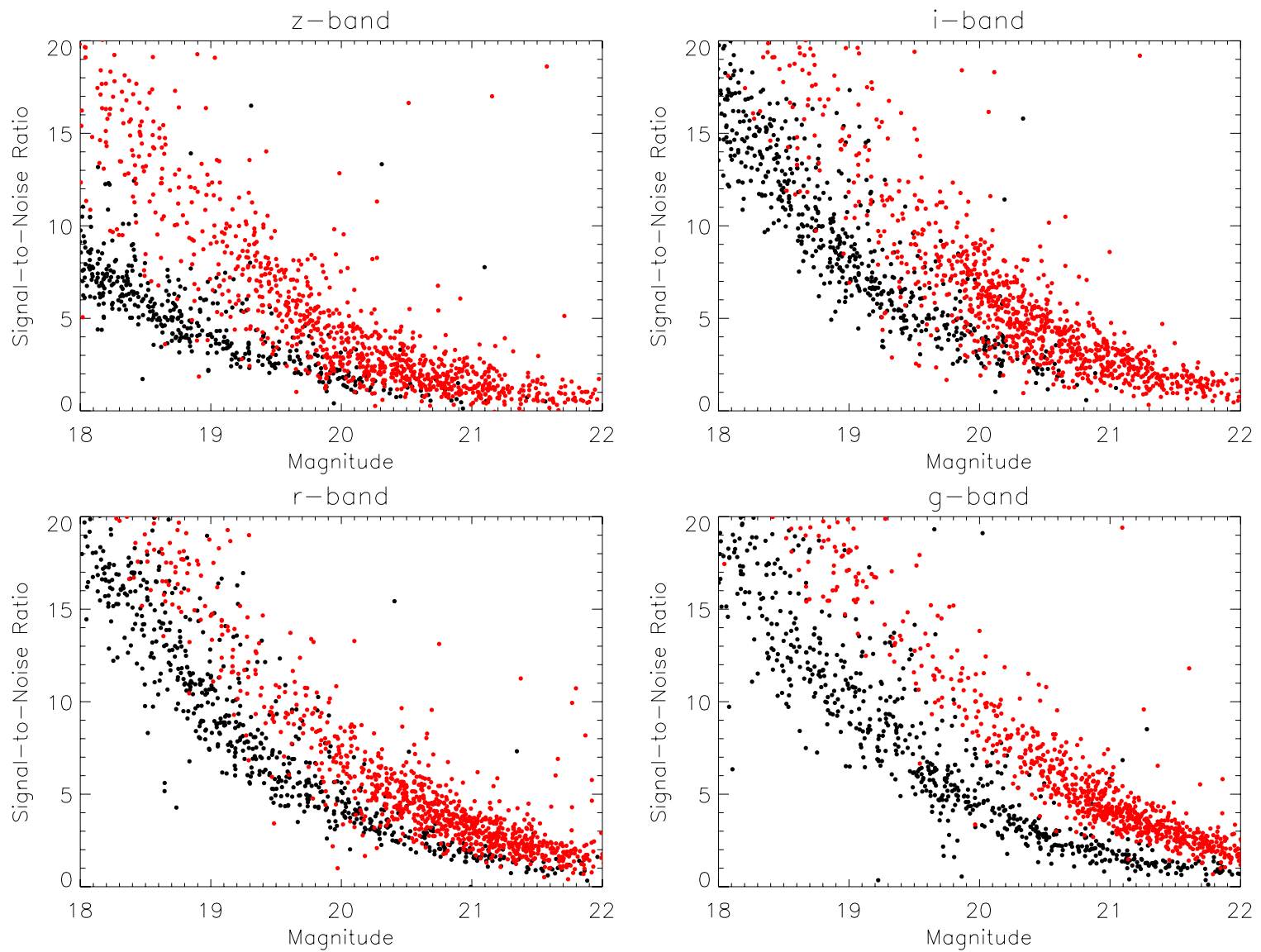

Figure 15. S/N per pixel as a function of extinction-corrected PSF magnitude in each of the four synthetic bandpass filters (griz) matching the photometric passbands of the SDSS imaging survey. In each case, the red dots represent BOSS data while the black dots represent SDSS data. The data were selected from a random sampling of objects classified as stars over the full SDSS and BOSS samples.

(A color version of this figure is available in the online journal.)

Table 7

DR9 Redshift Determination for Main Targets

\begin{tabular}{lccccr}
\hline \hline Sample & Unique Targets & Repeat Observations & Failed Redshifts & Purity & Contaminating Objects \\
\hline LOWZ & 103729 & 7646 & 119 & 102890 & 720 \\
CMASS & 324198 & 29493 & 4167 & 309307 & 10724 \\
Quasar & 154433 & 11601 & 31945 & 79570 & 42918 \\
\hline
\end{tabular}

all successfully classified main galaxies and quasars is shown in Figure 14.

\subsection{BOSS in the Context of Previous SDSS Spectroscopy}

As discussed in Section 1, the improvements to the BOSS spectrograph and increased cosmological volume of BOSS produce a new sample that is both complementary and a significant expansion beyond SDSS. The SDSS-I and II surveys obtained roughly 1.8 million spectra of galaxies, stars, and quasars (DR8: Aihara et al. 2011a). There are now about as many galaxies in the BOSS LOWZ sample as in the LRG sample used to derive the BAO constraints from SDSS, while the sample of CMASS galaxies represents a probe of an entirely new cosmological volume. The SDSS sample consists of more than 100,000 quasar spectra, with a median redshift of $z \sim 1.5$ and absolute magnitudes $\mathbf{M}_{i}<-22.0$ (Schneider et al. 2010). Just over 19,000 of these quasars lie beyond $z=2.15$; the new combined sample of Ly $\alpha$ quasars is already a factor 3.7 larger than what was available with SDSS.
BOSS produces more objects at high redshift largely due to the improved throughout of the spectrographs and the decreased sky background from the smaller fibers. The improved sensitivity is demonstrated through a comparison of the typical $\mathrm{S} / \mathrm{N}$ in BOSS spectra relative to SDSS. By comparing the quality of data from a random sampling of stellar point sources from both samples, we find roughly a factor of two improvement in $\mathrm{S} / \mathrm{N}$ per pixel in BOSS. An $\mathrm{S} / \mathrm{N}$ versus magnitude scatter plot of individual objects measured in the synthetic bandpass filters griz is shown in Figure 15. We do not include the synthetic $u$ filter because of the significant increase in the wavelength coverage of BOSS in that wavelength range.

The ambitious extension to redshifts beyond $z=0.6$ for the CMASS sample was made possible not only by improvements to the spectrographs, but also by the proven reliability of the idlspec2D pipeline to classify objects with spectra as low as $\mathrm{S} / \mathrm{N} \sim 2$ per co-added pixel in SDSS. An example of a successful classification of a faint SDSS LRG is shown in Figure 16. For comparison, a CMASS galaxy with reliable redshift is shown in the same figure. The BOSS galaxy has about 

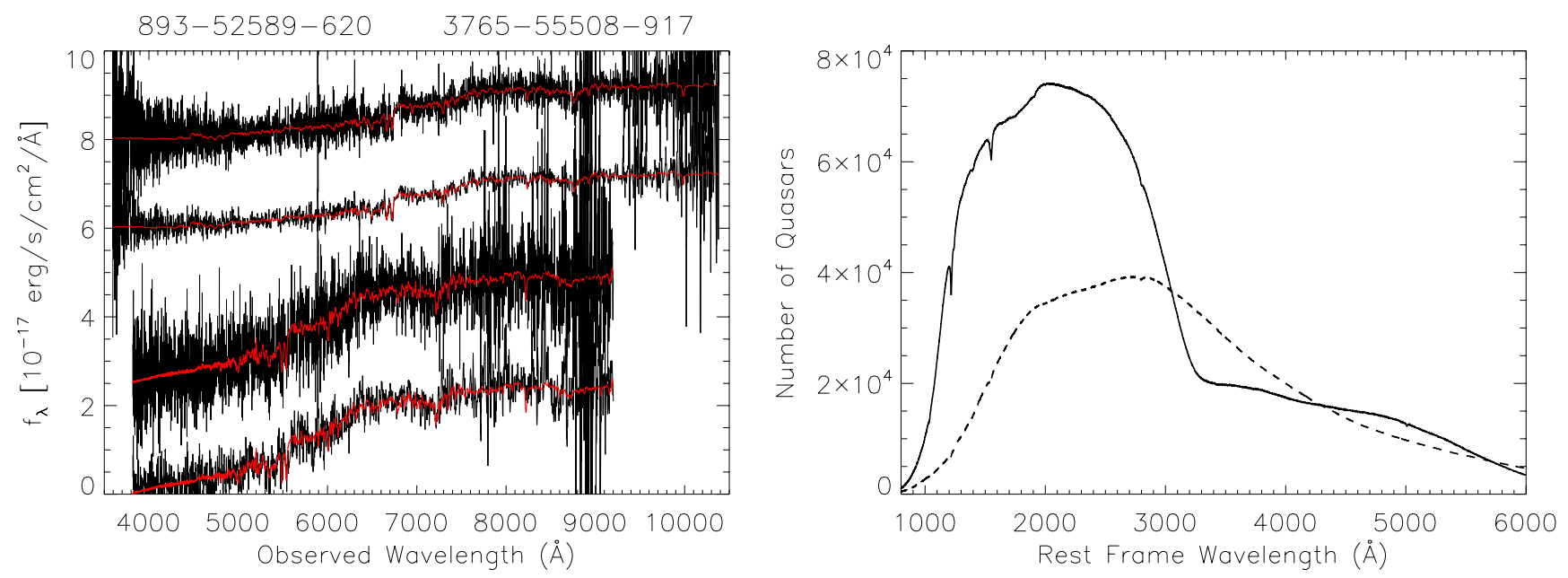

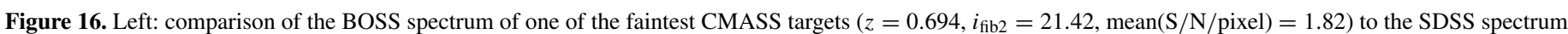

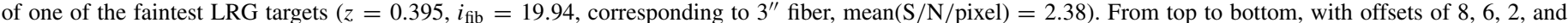

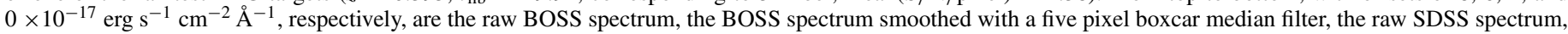

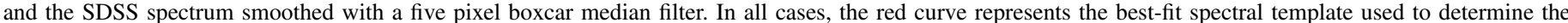

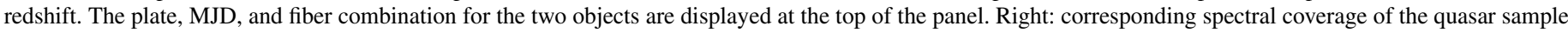
in BOSS (solid curve) relative to SDSS (dashed curve). The histogram represents the number of objects sampled at each rest-frame wavelength.

(A color version of this figure is available in the online journal.)

the same $\mathrm{S} / \mathrm{N}$, but has almost 1.5 magnitudes less flux entering the fiber.

As important as increasing the number of $\operatorname{Ly} \alpha$ quasars is the superior wavelength coverage of BOSS relative to SDSS. The expanded coverage from $\lambda \gtrsim 3780 \AA$ to $\lambda \gtrsim 3615 \AA$ makes the redshift range $2.15<z<2.3$ more accessible for Ly $\alpha$ forest studies. As shown in Figure 14, $19 \%$ of the Ly $\alpha$ quasars for BOSS lie between $2.15<z<2.3$. Figure 16 shows an increase in the number of observations at each wavelength in the Ly $\alpha$ forest for BOSS relative to SDSS.

\subsection{Early Science Results from BOSS Data}

In addition to the technical papers summarized in Section 1, members of the SDSS-III collaboration have published a number of papers using the BOSS data. While cosmology was the primary driver, the size and quality of the data sample enable many other studies of galaxy evolution and quasar physics.

Chen et al. (2012) applied principal component analysis (PCA) to the rest-frame 3700-5500 Å spectral region to estimate the properties of the BOSS galaxies. They find the fraction of galaxies with recent star formation in the CMASS sample first declines with increasing stellar mass, but flattens above a stellar mass of $10^{11.5} M_{\odot}$ at $z \sim 0.6$. This is in contrast with $z \sim 0.1$, where the fraction of galaxies with recent star formation declines monotonically with stellar mass (right panel of Figure 16 in Chen et al. 2012). The PCA fits as well as galaxies properties, such as stellar masses and stellar velocity dispersions, are included as part of the DR9 catalog. Shu et al. (2012) present the velocity-dispersion probability function for each galaxy in the LOWZ and CMASS samples. Correlating velocity dispersion with absolute magnitude and redshift, they find that galaxies of constant mass exhibit higher intrinsic scatter in velocity dispersion at higher redshifts. Maraston et al. (2012) present the first BOSS galaxy mass functions at redshift $0.4<z<0.7$ using the BOSS spectroscopic redshifts and ugriz photometry. A comparison to galaxy formation models shows that data-derived mass functions compare well with models at the typical BOSS redshift ( $z=0.55)$, even at the high mass end. However, the data do not show the evolution to zero redshift that is predicted in models of hierarchical mass build-up. The BOSS spectroscopy and SDSS imaging data has also been used to study the luminosity functions of galaxies surrounding LRGs (Tal et al. 2012). The data require a multiple component fit to produce a luminosity function consistent with the distribution of central galaxies and the most luminous satellite galaxies. The result stands in contrast with models that predict mass growth of central galaxies through major mergers.

Many observations with the Hubble Space Telescope (HST) have been taken inside the BOSS footprint. These high spatial resolution data offer a counterpart to the BOSS spectra, allowing, for example, a study of the morphology and size of the CMASS sample of galaxies (Masters et al. 2011). The study reveals that the majority of CMASS galaxies $(74 \% \pm 6 \%)$ exhibit early-type morphology as intended in the target selection algorithms. An additional color cut excluding galaxies with $g-i<2.35$ increases the population of early-type galaxies to $\geqslant 90 \%$, producing a subsample that is similar to that of the SDSS-I and II LRG galaxies. In an approach similar to that from the Sloan Lens Advanced Camera for Surveys (SLACS; Bolton et al. 2006, 2008; Auger et al. 2009), Brownstein et al. (2012) present a catalog of strong galaxy-galaxy gravitational lens systems discovered spectroscopically in the CMASS sample and observed with HST. Known as the BOSS Emission-Line Lensing Survey (BELLS), the HST imaging and BOSS spectra enable a reconstruction of the mass profile of the lensing galaxies. Bolton et al. (2012a) perform a joint analysis on the SLACS and BELLS samples and determine that galaxies tend to have steeper mass profiles with decreasing redshift. Unlike the studies of Tal et al. (2012), the results indicate that major dry mergers play a major role in the evolution of massive galaxies.

Finally, highlighting the potential for multi-wavelength studies, the first detection (Hand et al. 2012) of the kinematic Sunyaev-Zel'dovich effect (Sunyaev \& Zel'dovich 1972) was discovered in a correlation of BOSS galaxies with maps made by the Atacama Cosmology Telescope (Hincks et al. 2010). This is surely the first such result in a stream of studies that will combine BOSS spectra with data from projects such as WISE, XMM, Chandra, and Planck. 
Results from the main quasar sample are now also beginning to appear. The first such study constrains the two-point correlation function over the redshift range $2.2<z<2.8$ (White et al. 2012). The redshift space correlation function of quasars is described by a power-law that requires a bias factor of $b \sim 3.5$ for the CORE sample, implying that these quasars reside in halos with a typical mass of $\sim 10^{12} M_{\odot}$. Members of the BOSS collaboration will soon release results that constrain the quasar luminosity function, determine a composite quasar spectrum (as in Vanden Berk et al. 2001), and report variable absorption in BAL quasars, in addition to many other studies of quasar physics. In addition to results from the primary BOSS galaxy and quasar targets, analysis of data from the ancillary programs described in Appendix B continues and will result in an even more diverse array of findings than would be possible with the main samples alone.

\subsection{BOSS Cosmology}

As explained in Section 2.1, we intend to complete spectroscopy with BOSS over the $\sim 10,000 \mathrm{deg}^{2}$ footprint that falls at high Galactic latitude and was imaged by SDSS-I and II. The primary goal of the galaxy redshift survey is to achieve BAO distance scale constraints that are close to the limit set by cosmic variance out to $z=0.6$; the only substantial (factor-of-two) improvement possible at these redshifts would be to cover the remaining $3 \pi$ steradians of the sky. The BOSS Ly $\alpha$ forest survey will pioneer a new method of measuring three-dimensional structure in the high-redshift universe and provide the first BAO measurements of distance and expansion rate at $z>2$.

The first small scale clustering statistics of the LOWZ sample from the BOSS data are reported in Parejko et al. (2012). Measurements of clustering in the CMASS sample from the first year of BOSS data are found in White et al. (2010). That analysis includes constraints on the halo occupation distribution. With the full DR9 sample, the BOSS collaboration carried out a series of more ambitious clustering analysis. For example, Reid et al. (2012) measure the growth of structure and expansion rate at $z=0.57$ from anisotropic clustering, and Sánchez et al. (2012) explore the cosmological implications of the large-scale twopoint correlation function. The analysis for these cosmology studies shares much of the same framework as the galaxy BAO study. Toward that goal, Manera et al. (2012) generate a large sample of mock galaxy catalogs to characterize the statistics of large-scale structure, Ross et al. (2012a) present an analysis of potential systematics in the galaxy sample, and Tojeiro et al. (2012) measure structure growth using passive galaxies.

The efforts described in the previous paragraph made possible the first BOSS BAO measurement (Anderson et al. 2012). Using 264,283 CMASS galaxies over $3275 \mathrm{deg}^{2}$ and a redshift range $0.43<z<0.7$, we determined the angle-averaged galaxy correlation function and galaxy power spectrum in an effective volume of more than two $\mathrm{Gpc}^{3}$, the largest sample of the universe ever surveyed at this density. In combination with the SDSS LRG sample, the BAO detection provides a $1.7 \%$ measurement of the distance to $z=0.57$, the most precise distance constraint ever obtained from a galaxy survey. The resulting constraints on various cosmological models continue to support a flat Universe dominated by a cosmological constant.

Measurements of the three-dimensional correlation function using the first year of BOSS Ly $\alpha$ spectra are found in Slosar et al. (2011). This analysis also reports the first detection of flux correlations across widely separated sight lines, a measurement of redshift-distortion due to peculiar velocities, and constraints on the linear bias parameter of optical depth. The complete five year quasar sample should provide the means to extend the Slosar et al. (2011) analysis and observe the BAO feature at $z>2$ for the first time.

As described in Eisenstein et al. (2011), we have used a Fisher matrix formalism to predict the BAO constraints from the five year BOSS galaxy and Ly $\alpha$ forest surveys (drawing on Seo \& Eisenstein 2007; McDonald \& Eisenstein 2007), and to predict the impact of these constraints on cosmological parameter determinations. For the galaxy survey we forecast $1 \sigma$ precision of $1.0 \%$ on $D_{A}(z)$ and $1.8 \%$ on $H(z)$ at $z=0.35$ (bin width $0.2<z<0.5)$ and corresponding errors of $1.0 \%$ and $1.7 \%$ at $z=0.6$ (bin width $0.5<z<0.7$ ). Errors at the two redshifts are essentially uncorrelated, while the errors on $D_{A}(z)$ and $H(z)$ at a given redshift are correlated at the $40 \%$ level. Dividing into smaller bins would yield a finer grained view of $w(z)$ evolution, with larger errors per bin but the same aggregate precision. For the Ly $\alpha$ forest we update the forecast slightly from Eisenstein et al. (2011), predicting a measurement precision of $1.9 \%$ at a redshift approximately $z=2.5$ on an overall dilation factor scaling both $D_{A}(z)$ and $H^{-1}(z)$. This result was confirmed with a full simulation of the survey using mock quasar spectra (Le Goff et al. 2011). $D_{A}(z)$ and $H(z)$ have a higher degree of degeneracy than in the galaxy sample, with a correlation coefficient of 0.6; we therefore do not report projections for individual constraints here. In detail, Ly $\alpha$ forest projections depend on the level of clustering bias and redshift-space distortion in the forest, which are only now being measured precisely in ongoing analyses of the DR9 quasar sample.

The impact of these measurements on cosmological parameter determinations depends on the assumptions about external constraints from other data sets and about the underlying space of theoretical models. We have computed forecasts (Appendix A of Eisenstein et al. 2011) within the framework adopted by the DETF (Albrecht et al. 2006), which assumes an inflationary cold dark matter cosmology, allows departures from spatial flatness, and employs a dark energy equation of state described by $w(a)=w_{p}+w_{a}\left(a_{p}-a\right)$, where $a=(1+z)^{-1}$ and $w_{p}=w\left(z_{p}\right)$ is the value of $w$ at the "pivot" redshift $z_{p} \approx 0.5$ where it is best constrained by BOSS. When combining the forecast BOSS BAO constraints with Planck priors for CMB anisotropy measurements and "Stage II" priors that approximately describe the constraints from existing dark energy experiments, we project $1 \sigma$ errors of 0.030 on $w_{p}, 0.320$ on $w_{a}, 2 \times 10^{-3}$ on the curvature parameter $\Omega_{k}$, and $0.9 \%$ on the Hubble constant $H_{0}$. Combining BOSS data with measurements from other, upcoming "Stage III" experiments, such as supernova, weak lensing, and cluster abundance data from the Dark Energy Survey, will further tighten these constraints, breaking parameter degeneracies and allowing cross-checks of results from different methods. Within BOSS itself, analyses that exploit the full shape and redshift-space anisotropy of the galaxy correlation function or power spectrum (e.g.; Sánchez et al. 2012; Reid et al. 2012), or that use other statistical approaches sensitive to higher order correlations, can achieve tighter constraints on dark energy and curvature, can test whether the rate of cosmic structure growth is consistent with the predictions of General Relativity, and provide sensitivity to neutrino masses, inflation physics, extra radiation backgrounds, and other departures from the simplest forms of the $\Lambda$ CDM scenario. These approaches make stronger demands on theoretical models of non-linear gravitational evolution and galaxy bias than does BAO, but the BOSS clustering measurements allow detailed, high-precision tests of these models. 
The success of BOSS, as documented in this paper, should be viewed as strong encouragement to future efforts in constraining BAO over large volumes and large redshifts. BOSS data will have broad impact on our understanding of large-scale structure, massive galaxy evolution, quasar evolution, and the intergalactic medium. The ultimate impact on fundamental cosmology depends in part on what nature has in store. Any clearly detected deviations from a flat universe with a cosmological constant would have profound implications, taking us closer to understanding one of the most remarkable scientific discoveries of our time.

Funding for SDSS-III has been provided by the Alfred P. Sloan Foundation, the Participating Institutions, the National Science Foundation, and the U.S. Department of Energy Office of Science. The SDSS-III Web site is http://www.sdss3.org/.

SDSS-III is managed by the Astrophysical Research Consortium for the Participating Institutions of the SDSS-III Collaboration including the University of Arizona, the Brazilian Participation Group, Brookhaven National Laboratory, University of Cambridge, Carnegie Mellon University, University of Florida, the French Participation Group, the German Participation Group, Harvard University, the Instituto de Astrofisica de Canarias, the Michigan State/Notre Dame/JINA Participation Group, Johns Hopkins University, Lawrence Berkeley National Laboratory, Max Planck Institute for Astrophysics, Max Planck Institute for Extraterrestrial Physics, New Mexico State University, New York University, Ohio State University, Pennsylvania State University, University of Portsmouth, Princeton University, the Spanish Participation Group, University of Tokyo, University of Utah, Vanderbilt University, University of Virginia, University of Washington, and Yale University.

\section{APPENDIX A}

\section{FLAGS FOR PRIMARY TARGET CLASSES}

The algorithms for targeting various classes of galaxy and quasar targets described in Section 2 and several additional classes of object for calibration are all recorded in the BOSS_TARGET1 flag bit. A brief description of those objects is found below. The density and survey area containing each type of object is found in Table 8. The BOSS_TARGET2 keyword serves as a placeholder for additional targets, but has not yet been used in SDSS-III.

Galaxy target selection. As described in Section 2.3, the galaxy sample used to derive new BAO constraints was divided into two principal samples based on redshift. The LOWZ sample (represented by the flag GAL_LOZ) targets the redshift interval $0.15<z<0.43$ while the CMASS sample targets the redshift interval $0.43<z<0.7$. The CMASS sample is divided into the following four subsamples of galaxies, with significant overlap between several subsets.

1. GAL_CMASS is the core of the high redshift BOSS galaxy sample, with selection criteria described in the main text. The flag was assigned to a test sample in the BOSS commissioning data found in boss 1 and boss 2 and should not be used in those chunks. It was assigned to the full sample of CMASS objects starting in boss 3 .

2. GAL_CMASS_COMM represent galaxies that were targeted using a commissioning selection algorithm to investigate possible targeting strategies. Objects were chosen so that $d_{\perp}>0.55, i<20.14+1.6\left(d_{\perp}-0.8\right), 17.5<i<20.0$
Table 8 BOSS Programs with BOSS_TARGET1 Flag

\begin{tabular}{|c|c|c|c|}
\hline Program & $\begin{array}{c}\text { Bit } \\
\text { Number }\end{array}$ & $\begin{array}{l}\text { Density } \\
\left(\operatorname{deg}^{-2}\right)\end{array}$ & $\begin{array}{c}\text { Survey Area } \\
\left(\mathrm{deg}^{2}\right)\end{array}$ \\
\hline GAL_LOZ & 0 & $20-30$ & 10000 \\
\hline GAL_CMASS & 1 & 110 & 9750 \\
\hline GAL_CMASS_COMM & 2 & 115 & 500 \\
\hline GAL_CMASS_SPARSE & 3 & 5 & 9750 \\
\hline GAL_CMASS_ALL & 7 & 115 & 9750 \\
\hline QSO_CORE & 10 & 14 & $\sim 250$ \\
\hline QSO_BONUS & 11 & 17 & $\sim 250$ \\
\hline QSO_KNOWN_MIDZ & 12 & 1.5 & 10000 \\
\hline QSO_KNOWN_LOHIZ & 13 & 0.25 & $\sim 100$ \\
\hline QSO_NN & 14 & 20 & 10000 \\
\hline QSO_UKIDSS & 15 & 1.0 & $\sim 50$ \\
\hline QSO_KDE_COADD & 16 & 27 & $\sim 50$ \\
\hline QSO_LIKE & 17 & 28 & 10000 \\
\hline QSO_FIRST_BOSS & 18 & 1.0 & 10000 \\
\hline QSO_KDE & 19 & 28 & 10000 \\
\hline STD_FSTAR & 20 & 4.6 & 10000 \\
\hline TEMPLATE_GAL_PHOTO & 32 & 4.2 & $\sim 50$ \\
\hline TEMPLATE_QSO_SDSS & 33 & 2.6 & $\sim 50$ \\
\hline TEMPLATE_STAR_PHOTO & 34 & 3.1 & $\sim 50$ \\
\hline TEMPLATE_STAR_SPECTRO & 35 & 1.2 & $\sim 50$ \\
\hline QSO_CORE_MAIN & 40 & 20 & 10000 \\
\hline QSO_BONUS_MAIN & 41 & 19 & 10000 \\
\hline QSO_CORE_ED & 42 & 10 & 9000 \\
\hline QSO_CORE_LIKE & 43 & 11 & 9000 \\
\hline QSO_KNOWN_SUPPZ & 44 & 0.25 & $\sim 100$ \\
\hline
\end{tabular}

and $r-i<2$ where $r$ and $i$ are cmodel magnitudes. Additionally, objects were required to have $i_{\text {fib2 }}<22$ and $i_{\mathrm{PSF}}-i_{\text {model }}>0.2+0.2\left(20.0-i_{\text {model }}\right)$. This sample is complete only for the first two BOSS commissioning chunks, boss 1 and boss2. The flag was never phased out and appears in DR9 from objects in later stages in the tiling, representing a subset of galaxies that would otherwise satisfy the GAL_CMASS_COMM criteria. It should not be used for boss 3 or any chunks thereafter.

3. GAL_CMASS_SPARSE targets have a color-magnitude cut that is shifted relative to GAL_CMASS to $i<$ $20.14+1.6 \times\left(d_{\perp}-0.8\right)$ to allow for completeness studies. Galaxies are either flagged GAL_CMASS or GAL_CMASS_SPARSE, but not both. This flag was introduced in boss3, after the commissioning period for galaxy target selection.

4. GAL_CMASS_ALL is used internally in the target selection code, representing the union of the GAL_CMASS and GAL_CMASS_SPARSE targets for boss3 and thereafter. Because the sample of galaxies identified by this flag can be reconstructed by the union of GAL_CMASS and GAL_CMASS_SPARSE, it provides no additional information and should rarely be needed by the user.

Quasar target selection. The use of quasar target flags evolved significantly throughout the first two years of the survey as different algorithms were tested to maximize the targeting efficiency. All programs with the QSO_prefix in Table 8 were targeted as potential quasars. A detailed explanation of each program is given in Ross et al. (2012b); we simply report the densities and survey area for each program. A few target classes bear special mention.

1. The QSO_UKIDSS and QSO_KDE_COADD target classes were only used in the boss 1 chunk of commissioning 
data on Stripe 82 , covered by a small number of plates between $0^{\circ}<\alpha<40^{\circ}$.

2. The QSO_CORE_ED and QSO_CORE_LIKE classes were implemented in the second year.

3. The QSO_CORE and QSO_BONUS classes were deprecated after the boss2 chunk and replaced by the QSO_CORE_MAIN and QSO_BONUS_MAIN classes for the rest of the survey, beginning with chunk3.

Calibration programs. There are five target classes dedicated to calibration programs within BOSS. The most prevalent target flag is STD_FSTAR, used to denote objects chosen as likely F stars as described in Section 3.2. These objects are used both for flux calibration and correction for telluric absorption. The other four target classes were only used in the Stripe 82 commissioning plates in the boss 1 chunk. These contain objects to aid in the development of templates for classification and redshift determination (Bolton et al. 2012b). In order for the classification to be as robust and informative as possible, the set of template spectra were selected to cover a wide range in the space of intrinsic spectral variation. Several classes contain objects previously observed in SDSS; the extended wavelength coverage of BOSS requires repeat spectra of these objects to cover 361-1014 $\mathrm{nm}$ in the observer frame. The targets are divided into four categories.

1. TEMPLATE_GAL_PHOTO were galaxies selected to uniformly cover the space of $[(g-r),(r-i), i]$. Objects were first chosen with dereddened cmodelmag $i<20, g_{\text {fib }}$, $r_{\text {fib }}$, and $i_{\text {fib }}>16.5, \mathrm{~S} / \mathrm{N}>5.0$ for gri model magnitudes to limit scatter in color measurements, and an absolute value of the difference between the model magnitude and cmodel magnitude in $i$ less than 0.8 . Next, for each galaxy, the total number of galaxies within a radius of 0.1 magnitudes in $[(g-r),(r-i), i]$ space was computed and taken as an estimate of the local density in that space. A subsample was then drawn at random, with the probability of retaining any given draw proportional to the inverse of the local density. This generates an approximately uniform sampling of the populated region within the color-color-magnitude space, where colors $g-r$ and $r-i$ were defined using model magnitudes and $i$ was determined using cmodel magnitudes.

2. TEMPLATE_QSO_SDSS1 were selected from the DR7 catalog of quasars (Schneider et al. 2007). The sample is restricted to objects with dereddened PSF magnitudes in ugriz $<20.0$ and gri fiber magnitudes $>16.5$. A random subsample was chosen after imposing a uniform distribution in redshift out to $z<3.2$ and taking all quasars at redshifts $z>3.2$. The PCA redshift templates in BOSS are based exclusively on this sample.

3. TEMPLATE_STAR_PHOTO were selected in similar fashion to the galaxy sample above, with dereddened PSF magnitudes brighter than 19.25 in any one of ugriz, $\mathrm{S} / \mathrm{N}>5.0$ in all filters using PSF magnitudes, and fiber magnitudes fainter than 16.5 in each of gri. A local density estimator within the PSF magnitude space of $[(u-g)$, $(g-r),(r-i),(i-z)]$ was computed from the number of stars within a 4 -sphere of radius 0.1 magnitude centered on each star. A random subsample of stars was drawn with the probability of retaining any given draw proportional to the local density to the power -0.7 (compared to -1 for the galaxy sample). This heuristic factor is chosen to retain some preferential weighting in favor of the core stellar locus, while still achieving significant representation from the less densely populated regions of color space.
4. TEMPLATE_STAR_SPECTRO were selected from the SDSS (non-SEGUE) spectroscopic database for reobservation to provide a sample of spectra that cover the major classes of stellar objects that lie away from the main stellar locus. The objects were required to have been both targeted as stars photometrically and classified as stars spectroscopically. The objects are required to have dereddened PSF magnitudes brighter than 19.25 in ugriz and fiber magnitudes fainter than 16.5 in each of gri. Stars were then selected at random to represent each of the following SDSS target categories identified by the DR9 LEGACY_TARGET1 ${ }^{82}$ and LEGACY_TARGET2 ${ }^{83}$ flags: STAR_BHB, STAR_CARBON, STĀR_BROWN_ DWARF, STAR_SUB_DWARF, STAR_CATY_VAR, STAR_RED_DWARF, STAR_WHITE_DWARF, STAR_PN, REDDEN_STD, SPECTROPHOTO_STD, and HOT_STD.

\section{APPENDIX B}

\section{TARGET SELECTION AND SCIENTIFIC MOTIVATION FOR ANCILLARY SCIENCE PROGRAMS}

The first category of ancillary science programs lies in the region of Stripe 82 (the celestial equator in the SGC) that has completed observations and will be included in DR9. The second set includes programs that are dispersed throughout the full BOSS footprint and will continue to accumulate data throughout the survey. Additional ancillary programs may be added in the future; those programs will be described in future data release papers. All ancillary targets are assigned fibers at a priority that is lower than the primary galaxy and quasar targets; sample selection for these programs is therefore not complete. Here we provide a comprehensive description of the motivation, target selection, and the target densities for these programs. For programs that are divided into subprograms, we also provide a description of the ancillary target flags and selection for each subprogram. The target bits and statistics for the ancillary science targets are reported in Tables 9 and 10. The target bit names appear in bold font in what follows.

\section{B.1. Stripe 82}

Several of these BOSS ancillary programs are contained completely in the Stripe 82 region. We finished all plates in Stripe 82 in 2011, leading to a group of ancillary programs that are included in its entirety in DR9. Starting with the nearest objects, we explain the rationale and target selection behind each of these programs below.

The transient universe through Stripe 82. The repeat imaging of Stripe 82 allows identification of transient and variable phenomena of all sorts (for example Anderson et al. 2008; Blake et al. 2008; Becker et al. 2008; Kowalski et al. 2009; Bhatti et al. 2010; Becker et al. 2011; Sako et al. 2011). In this program, several classes of variable point sources and highproper motion stars discovered in Stripe 82 photometry were targeted for spectroscopy. These objects include flaring M stars (Kowalski et al. 2009), faint high proper motion stars (Scholz et al. 2009; Schmidt et al. 2010c), candidate low-metallicity M dwarfs, and diverse samples of variable stars (Roelofs et al. 2007; Anderson et al. 2008; Blake et al. 2008). BOSS spectra were obtained to characterize the statistical properties of these

\footnotetext{
82 http://www.sdss3.org/dr9/algorithms/bitmask_legacy_target1.php

83 http://www.sdss3.org/dr9/algorithms/bitmask_legacy_target2.php
} 
Table 9

BOSS Ancillary Programs with ANCILLARY_TARGET1 Flag

\begin{tabular}{|c|c|c|c|c|}
\hline Primary Program & SubProgram & $\begin{array}{c}\text { Bit } \\
\text { Number }\end{array}$ & $\begin{array}{l}\text { Density } \\
\left(\mathrm{deg}^{-2}\right)\end{array}$ & $\begin{array}{c}\text { Survey Area } \\
\left(\operatorname{deg}^{2}\right)\end{array}$ \\
\hline Transient Universe & $\mathrm{AMC}$ & 0 & 0.05 & $220^{\mathrm{a}}$ \\
\hline Transient Universe & FLARE1 & 1 & 0.2 & $220^{\mathrm{a}}$ \\
\hline Transient Universe & FLARE2 & 2 & 0.7 & $220^{\mathrm{a}}$ \\
\hline Transient Universe & HPM & 3 & 0.5 & $220^{\mathrm{a}}$ \\
\hline Transient Universe & LOW_MET & 4 & 0.3 & $220^{\mathrm{a}}$ \\
\hline Transient Universe & VARS & 5 & 0.9 & $220^{\mathrm{a}}$ \\
\hline High Energy Blazars & BLAZGVAR & 6 & $\ll 1$ & $7650^{\mathrm{b}}$ \\
\hline High Energy Blazars & BLAZR & 7 & $\ll 1$ & $7650^{\mathrm{b}}$ \\
\hline High Energy Blazars & BLAZGXR & 8 & $\ll 1$ & $7650^{\mathrm{b}}$ \\
\hline High Energy Blazars & BLAZXRSAM & 9 & $\ll 1$ & $7650^{\mathrm{b}}$ \\
\hline Remarkable X-ray Sources & XMMBRIGHT & 11 & 0.1 & $7650^{\mathrm{b}}$ \\
\hline Remarkable X-ray Sources & XMMGRIZ & 12 & 0.02 & $7650^{\mathrm{b}}$ \\
\hline Remarkable X-ray Sources & XMMHR & 13 & 0.1 & $7650^{\mathrm{b}}$ \\
\hline Remarkable X-ray Sources & XMMRED & 14 & 0.08 & $7650^{\mathrm{b}}$ \\
\hline BAL Variability & FBQSBAL & 15 & 0.003 & 5740 \\
\hline BAL Variability & LBQSBAL & 16 & 0.002 & 5740 \\
\hline BAL Variability & ODDBAL & 17 & 0.007 & 5740 \\
\hline BAL Variability & OTBAL & 18 & 0.003 & 5740 \\
\hline BAL Variability & PREVBAL & 19 & 0.004 & 5740 \\
\hline BAL Variability & VARBAL & 20 & 0.4 & 5740 \\
\hline Bright Galaxies & BRIGHTGAL & 21 & 1.1 & $7650^{\mathrm{b}}$ \\
\hline Variable Quasar Absorption & QSO_AAL & 22 & 0.08 & $7650^{\mathrm{b}}$ \\
\hline Variable Quasar Absorption & QSO_AALS & 23 & 0.2 & $7650^{\mathrm{b}}$ \\
\hline Variable Quasar Absorption & QSO_IAL & 24 & 0.05 & $7650^{\mathrm{b}}$ \\
\hline Variable Quasar Absorption & QSO_RADIO & 25 & 0.04 & $7650^{\mathrm{b}}$ \\
\hline Variable Quasar Absorption & QSO_RADIO_AAL & 26 & 0.02 & $7650^{\mathrm{b}}$ \\
\hline Variable Quasar Absorption & QSO_RADIO_IAL & 27 & 0.01 & $7650^{\mathrm{b}}$ \\
\hline Variable Quasar Absorption & QSO_NOAALS & 28 & 0.01 & $7650^{\mathrm{b}}$ \\
\hline High Redshift Quasars & QSO_GRI & 29 & 2.7 & $220^{\mathrm{a}}$ \\
\hline High Redshift Quasars & QSO_GRI & 29 & 0.8 & 2500 \\
\hline High Redshift Quasars & QSO_HIZ & 30 & 0.7 & $220^{\mathrm{a}}$ \\
\hline High Redshift Quasars & QSO_HIZ & 30 & 0.2 & 2500 \\
\hline High Redshift Quasars & QSO_RIZ & 31 & 1.2 & $220^{\mathrm{a}}$ \\
\hline High Redshift Quasars & QSO_RIZ & 31 & 0.6 & 2500 \\
\hline Reddened Quasars & RQSS_SF & 32 & 1.0 & $\sim 50$ \\
\hline Reddened Quasars & RQSS_SFC & 33 & 0.3 & $\sim 50$ \\
\hline Reddened Quasars & RQSS_STM & 34 & 0.2 & $\sim 50$ \\
\hline Reddened Quasars & RQSS_STMC & 35 & 0.1 & $\sim 50$ \\
\hline SN Host Galaxies & SN_GAL1 & 36 & 13.8 & $220^{\mathrm{a}}$ \\
\hline SN Host Galaxies & SN_GAL2 & 37 & 0.4 & $220^{\mathrm{a}}$ \\
\hline SN Host Galaxies & SN_GAL3 & 38 & 0.1 & $220^{\mathrm{a}}$ \\
\hline SN Host Galaxies & SN_LOC & 39 & 1.6 & $220^{\mathrm{a}}$ \\
\hline SN Host Galaxies & SPEC_SN & 40 & 0.009 & $220^{\mathrm{a}}$ \\
\hline Low Mass Binary Stars & SPOKE & 41 & 0.1 & $7650^{\mathrm{b}}$ \\
\hline White Dwarf Stars & WHITEDWARF_NEW & 42 & 0.5 & $7430^{\mathrm{b}}$ \\
\hline White Dwarf Stars & WHITEDWARF_SDSS & 43 & 0.5 & $7430^{\mathrm{b}}$ \\
\hline Very Low Mass Stars & BRIGHTERL & 44 & 0.07 & $220^{\mathrm{a}}$ \\
\hline Very Low Mass Stars & BRIGHTERL & 44 & 0.08 & $7430^{\mathrm{b}}$ \\
\hline Very Low Mass Stars & BRIGHTERM & 45 & 2.9 & $220^{\mathrm{a}}$ \\
\hline Very Low Mass Stars & BRIGHTERM & 45 & 0.3 & $7430^{\mathrm{b}}$ \\
\hline Very Low Mass Stars & FAINTERL & 46 & 0.3 & $220^{\mathrm{a}}$ \\
\hline Very Low Mass Stars & FAINTERL & 46 & 0.2 & $7430^{\mathrm{b}}$ \\
\hline Very Low Mass Stars & FAINTERM & 47 & 2.2 & $220^{\mathrm{a}}$ \\
\hline Very Low Mass Stars & FAINTERM & 47 & 0.6 & $7430^{\mathrm{b}}$ \\
\hline Distant Halo Giants & RED_KG & 48 & 0.8 & 10,000 \\
\hline Distant Halo Giants & RVTEST & 49 & $\sim 0.8$ & $\sim 50$ \\
\hline High Energy Blazars & BLAZGRFLAT & 50 & 0.02 & $7650^{\mathrm{b}}$ \\
\hline High Energy Blazars & BLAZGRQSO & 51 & 0.02 & $7650^{\mathrm{b}}$ \\
\hline High Energy Blazars & BLAZGX & 52 & 0.01 & $7650^{\mathrm{b}}$ \\
\hline High Energy Blazars & BLAZGXQSO & 53 & 0.01 & $7650^{\mathrm{b}}$ \\
\hline High Energy Blazars & BLAZGXR & 54 & 0.03 & $7650^{\mathrm{b}}$ \\
\hline High Energy Blazars & BLAZXR & 55 & 0.1 & $7650^{\mathrm{b}}$ \\
\hline Star Forming Radio Galaxies & BLUE_RADIO & 56 & 0.4 & 10,000 \\
\hline X-ray View of Star-Formation and Accretion & CHANDRAV1 & 57 & 0.2 & $7650^{\mathrm{b}}$ \\
\hline
\end{tabular}


Table 9

(Continued)

\begin{tabular}{lcccc}
\hline \hline Primary Program & SubProgram & $\begin{array}{c}\text { Bit } \\
\text { Number }\end{array}$ & $\begin{array}{c}\text { Density } \\
\left(\mathrm{deg}^{-2}\right)\end{array}$ & $\begin{array}{c}\text { Survey Area } \\
\left(\mathrm{deg}^{2}\right)\end{array}$ \\
\hline Remarkable X-ray Sources & CXOBRIGHT & 58 & 0.05 & $7650^{\mathrm{b}}$ \\
Remarkable X-ray Sources & CXOGRIZ & 59 & 0.009 & $7650^{\mathrm{b}}$ \\
Remarkable X-ray Sources & CXORED & 60 & 0.08 & $7650^{\mathrm{b}}$ \\
Luminous Blue Galaxies & ELG & 61 & 22 & 143 \\
Galaxies near Quasar Sight Lines & GAL_NEAR_QSO & 62 & 0.3 & $7650^{\mathrm{b}}$ \\
Transient Universe & MTEMP & 63 & 0.5 & $220^{\mathrm{a}}$ \\
\hline
\end{tabular}

Notes.

${ }^{\text {a }}$ Sample is taken from the Stripe 82 region.

${ }^{\mathrm{b}}$ Sample is taken from BOSS footprint that overlaps with DR7 imaging data.

Table 10

BOSS Ancillary Programs with ANCILLARY_TARGET2 Flag

\begin{tabular}{lcccc}
\hline \hline Primary Program & SubProgram & $\begin{array}{c}\text { Bit } \\
\text { Number }\end{array}$ & $\begin{array}{c}\text { Density } \\
\left(\mathrm{deg}^{-2}\right)\end{array}$ & $\begin{array}{c}\text { Survey Area } \\
\left(\mathrm{deg}^{2}\right)\end{array}$ \\
\hline High Redshift Quasars with UKIDSS & HIZQSO82 & 0 & 0.5 & $220^{\mathrm{a}}$ \\
High Redshift Quasars with UKIDSS & HIZQSOIR & 1 & 0.3 & 700 \\
$K$-band Selected Quasars & KQSO_BOSS & 2 & 1.0 & $220^{\mathrm{a}}$ \\
No Quasar Left Behind & QSO_VAR & 3 & 6.5 & $220^{\mathrm{a}}$ \\
Variability Selected Quasars & QSO_VAR_FPG & 4 & 3.4 & $220^{\mathrm{a}}$ \\
Double-lobed Radio Quasars & RADIO_2LOBE_QSO & 5 & 0.3 & 7650 \\
Brightest Cluster Galaxies & STRIPE82BCG & 6 & 6.0 & $220^{\mathrm{a}}$ \\
\hline
\end{tabular}

Note. ${ }^{\text {a }}$ Sample is taken from the Stripe 82 region.

categories of transient objects. Approximately 600 objects were targeted across Stripe 82. The classes of transient objects were selected as follows.

1. FLARE1, FLARE2 consists of $\sim 200$ flaring $M$ stars selected from the Bramich et al. (2008) and Ivezić et al. (2007) catalogs with $g_{\mathrm{PSF}}<21.4, i_{\mathrm{PSF}}<19,0.3<$ $\left(i_{\mathrm{PSF}}-z_{\mathrm{PSF}}\right)<1.3$, and exhibiting a flare event in the Stripe 82 imaging data with amplitude $\Delta u>1$ mag (Kowalski et al. 2009).

2. HPM consists of $\sim 100$ high proper motion stars also selected from the catalogs of Bramich et al. (2008) and Ivezić et al. (2007) with an emphasis on faint objects with high proper motions $\left(\mu>0.1\right.$ mas $\left.\mathrm{yr}^{-1}\right)$. The goal of this target selection was to identify nearby low-mass stars and white dwarfs. Candidate nearby low-mass stars include faint stars $\left(19<z_{\mathrm{PSF}}<20\right)$ with $\left(i_{\mathrm{PSF}}-z_{\mathrm{PSF}}\right)>1.5$, including objects with photometric detections in $z$ band only. Candidate nearby white dwarfs include stars with $\left(g_{\mathrm{PSF}}-r_{\mathrm{PSF}}\right) \sim 0$ and $g_{\mathrm{PSF}}>19$.

3. VARS consists of $\sim 200$ variables with $g_{\mathrm{PSF}}<21.5$, $\left(g_{\mathrm{PSF}}-r_{\mathrm{PSF}}\right)>-0.5$, and $\mathrm{rms}$ variability in $g$ band $>0.1 \mathrm{mag}$. These objects were selected to lie outside the stellar and quasar loci in $\left(g_{\mathrm{PSF}}-r_{\mathrm{PSF}}\right)-\left(r_{\mathrm{PSF}}-i_{\mathrm{PSF}}\right)$ colorcolor space as defined in Fan et al. (1999).

4. AMC consists of ten candidate Am CVn stars selected based on their variability and colors $\left(\left(u_{\mathrm{PSF}}-g_{\mathrm{PSF}}\right)>0.4\right.$ and $\left.\left(g_{\mathrm{PSF}}-r_{\mathrm{PSF}}\right)>0\right)$.

As part of this ancillary program, a number of spectral templates were observed as well. These include a random sample of approximately $100 \mathrm{M}$ stars, designated MTEMP, selected to span the spectra range M0 to M8 following the color criteria outlined in West et al. $\left(2005,17.5<i_{\mathrm{PSF}}<18.5\right.$, $\left.\left(r_{\mathrm{PSF}}-i_{\mathrm{PSF}}\right)>0.5,\left(i_{\mathrm{PSF}}-z_{\mathrm{PSF}}\right)>0.3\right)$. Approximately 70 candidate low-metallicity M stars (flag LOW_MET) were also targeted; their colors were selected to be slightly outside the low-mass star stellar locus defined in West et al. (2005). The purpose of these sub-samples is to enable direct comparisons between the prevalence of line emission in the photometricallyselected flare stars and field M stars selected only on the basis of their colors.

Host Galaxies of SDSS-II SNe. While many SNe Ia identified in the SDSS-II program (Frieman et al. 2008) were spectroscopically confirmed and used in the first year cosmology results (Kessler et al. 2009), most $\mathrm{SNe}$ and their host galaxies have not been observed spectroscopically. With redshifts of the host galaxies, SN light curves can be fit with fewer degrees of freedom, leading to identifications of SN type with higher confidence. The new classifications will allow a much larger number of SNe Ia to be placed on the Hubble diagram (Campbell et al. 2012). In this ancillary program, candidate $\mathrm{SN}$ hosts were drawn from a database containing 21,787 potentially variable objects (Sako et al. 2008) determined from the repeat imaging of Stripe 82. The next stage of selection required coincidence of a signal in at least two passbands after vetoing regions of bright stars and variability from known AGNs or variable stars. In total the list includes 4099 candidates of different SN types and different confidence levels as determined from a Bayesian classification (Sako et al. 2011) of the light curve shapes. Fibers were assigned to 3743 of these host galaxies to obtain a redshift and thereby improve the SN classification. Approximately one third of the targets have light curves that do not resemble $\mathrm{SNe}$ and are included as a control sample. The redshifts and new classification will lead to a nearly complete sample of SNe Ia to $z<0.4$ and an enhanced cosmological analysis. The new classification will also enable a large statistical study of the correlated properties between SNe Ia and their host galaxies (e.g., Kelly et al. 2010; Sullivan et al. 2010; Lampeitl et al. 2010; Brandt et al. 2010). Subsamples of SNe from this ancillary program have already demonstrated correlations of residuals from the Hubble 
Diagram with spectroscopically-derived host properties (D'Andrea et al. 2011). The candidates were selected after visual inspection and divided according to the following ancillary program flags: SN_GAL1: fibers assigned to the position of the core of the nearest candidate host galaxy (this accounted for 95\% of the cases); SN_GAL2: fibers assigned to the position of the core of the second nearest candidate host galaxy (when the first was clearly a background object or a misclassified star; $4 \%$ of the targets); SN_GAL3: fibers assigned to the position of the core of the third nearest candidate host galaxy (1\% of the targets); SN_LOC: already have SDSS host spectra so the fiber was assigned to the location of the original SN candidate; and SPEC_SN: SNe identified from SDSS spectra (e.g., Krughoff et al. 2011) rather than photometric variability.

Brightest cluster galaxies (BCGs). Over 3000 groups and clusters have been identified photometrically in Stripe 82 (Geach et al. 2011; Murphy et al. 2012). The clusters were selected from ugriz photometry generated from the co-addition of Stripe 82 images (Annis et al. 2011) that is $\sim 2$ mag deeper than the rest of the area in the BOSS footprint. These clusters have photometric redshifts in the range $0<z<0.6$ (median $z=0.32$ ) and are expected to reside in dark matter halos with masses in excess of $2.5 \times 10^{13} M_{\odot}$. Each cluster is assigned a "BCG," which is simply defined as the brightest member associated with the cluster detection. To confirm the cluster redshifts, we obtained spectra of the likely BCGs with magnitudes $17<i_{\text {fib2 }}<21.7$ and colors that vary with redshift according to the cluster detection algorithm (Murphy et al. 2012). One thousand five hundred and five galaxies were requested and 1345 were observed that were not included in the main SDSS-I or -II galaxy, SDSS LRG, BOSS LOWZ, BOSS CMASS, 2SLAQ, or WiggleZ samples. This new sample of spectroscopically-confirmed clusters will enable a wide variety of science including the weak lensing of groups and clusters, the link between star formation and AGN activity in BCGs, and the LRG population of dark matter halos.

High quality galaxy spectra. Two plates (3615 and 3647) are being observed repeatedly throughout the survey several times per year to test reproducibility of extracted spectral parameters and to create exceptionally high $\mathrm{S} / \mathrm{N}$ spectra of a subset of objects in the main BOSS sample. These observations will produce accumulated exposure times of more than $20 \mathrm{hr}$ on all objects on the plates, including 96 LOWZ and 503 CMASS galaxies at $\alpha=37^{\circ}$ on Stripe 82. These plates contain identical spectroscopic targets but are drilled for different airmass. The targets acquired $16.5 \mathrm{hr}$ of total exposure between 2009 December 1 and 2011 July 15 and will be observed in each of the three remaining years of the survey. The final observations will enable detailed spectral population studies of intermediate redshift galaxies. The data also provide a platform for tests of the consistency in spectroscopic parameter extraction (e.g., Shu et al. 2012; Thomas et al. 2012) and of systematic errors in the models of continuum fitting in the $\mathrm{Ly} \alpha$ forest region of the quasar sample (e.g., Lee et al. 2012).

No quasar left behind. We observed unresolved sources that had not been previously observed in SDSS spectroscopy that exhibit photometric variability statistically similar to that of spectroscopically confirmed quasars. Approximately 1500 targets were selected from 11,000 variable sources with $16.2<$ $i_{\mathrm{PSF}}<20.5$ identified according to the technique outlined in Butler \& Bloom (2011). This study complements the sample of "Variability Selected Quasars" described below but targets brighter objects without color cuts, leading to a higher density of lower redshift quasars. The adopted variability-based selection criteria correctly identify $96 \%$ of previously known quasars, and more than $80 \%$ of targets are expected to be quasars in the redshift range $0<z<5$. The brightest of these $\left(i_{\mathrm{PSF}}<19\right)$ were observed to test the completeness of the color-selected SDSS sample (Ross et al. 2012b). The fainter subset represents a nearly complete sample that was selected from uniform photometry. In addition, the program provides an invaluable training sample for optimizing quasar selection algorithms based on photometric variability, vital for future synoptic surveys.

Variability selected quasars. Variability is used to improve the selection efficiency for quasars around $z=2.7$ and $\sim 3.5$, where they lie in the region of color-color space that is occupied by the stellar locus. The selection method is quite similar to that used for the variability target selection used to select the main sample of quasars in Stripe 82 as described at the end of Section 2.4. The sample also complements the sample of "No Quasar Left Behind" but includes fainter quasars and imposes a loose color selection to select primarily objects at $z>2.15$. In this method, objects were included with $i_{\text {fib } 2}>18,\left(g_{\mathrm{PSF}}-i_{\mathrm{PSF}}\right)<2.2$, $\left(u_{\mathrm{PSF}}-g_{\mathrm{PSF}}\right)>0.4$, and $c_{1}<1.5$ or $c_{3}<0$. Colors $c_{1}$ and $c_{3}$ are defined in Fan et al. (1999) as

$$
\begin{aligned}
& c_{1}=0.95(u-g)+0.31(g-r)+0.11(r-i), \\
& c_{3}=-0.39(u-g)+0.79(g-r)+0.47(r-i) .
\end{aligned}
$$

A variability neural network is used to quantify the likelihood that each object is a quasar. The neural network takes as an input (1) the $\chi^{2} \mathrm{~s}$ between the light curve in each band and a model assuming no variability and (2) a structure function derived from the light curve as in Schmidt et al. (2010a). Targets require a probability of being a quasar from the variability neural network greater than 0.95 . Candidate quasars are chosen from $\sim 60$ imaging epochs from the last ten years, resulting in about $15 \mathrm{deg}^{-2}$ quasar candidates. After removal of previously known quasars and removal of candidates that were already included in the main quasar selection, $3.4 \mathrm{deg}^{-2}$ were selected for this sample. These data address the completeness of color selections and identifies obscured objects that would not be selected otherwise (Palanque-Delabrouille et al. 2011). These data are also being used to demonstrate the efficiency of variability target selection for possible implementation in future surveys such as BigBOSS (Schlegel et al. 2011), a ground-based dark energy experiment to study BAO and the growth of structure with deeper observations than BOSS.

Reddened quasars. Quasar candidates likely to be intrinsically reddened with $E(B-V)>0.5$ were selected from SDSS photometry and either FIRST or the Two Micron All Sky Survey (2MASS; Skrutskie et al. 2006). The goal was a better determination of the distribution of reddenings in intervening absorbers and at the quasar redshift (e.g., Hopkins et al. 2004; Ménard et al. 2008), and investigating reddening as a function of quasar properties. Targets were selected based on color selection and stellar morphology. All targets were required to have $17<z_{\mathrm{PSF}}<21$ with Galactic extinction colors satisfying $\left(g_{\mathrm{PSF}}-i_{\mathrm{PSF}}\right)-3 \sigma_{\left(g_{\mathrm{PSF}}-i_{\mathrm{PSF}}\right)}>0.9$, where $\sigma$ is the measurement error in the color. Targets selected from SDSS plus FIRST were denoted RQSS_SF or RQSS_SFC (the trailing C indicates a CHILD object in SDSS, as in Stoughton et al. 2002) and were additionally required to match within $2^{\prime \prime}$ of a FIRST source. Targets selected from SDSS plus 2MASS (RQSS_TM or RQSS_TMC) were additionally required to match within $3^{\prime \prime}$ of a 2 MASS source having $11<K<15.1,(J-K)>1.25$, 
and a seven-dimensional color distance (Covey et al. 2007) greater than 50 (this is the distance in units of $\chi^{2}$ to the point on the stellar locus with the same $(g-i)$ color as the object). The program was discontinued after the first year due to low yield, and targets are included on only 13 completed plates. It is superceded by the program " $K$-band Limited Sample of Quasars."

K-band limited sample of quasars. Potential quasars are identified via photometering UKIDSS (Lawrence et al. 2007) at the position of SDSS sources to a limiting magnitude $K_{\mathrm{AB}}<19.0\left(K_{\text {vega }}<17.1\right)$. The intention was to identify highly red and reddened quasars not included in the main selection methods. The sample allows an investigation, for instance, of the incidence of BAL quasar samples, or of highly reddened gravitationally lensed quasars. In the original selection, approximately 2000 targets were identified that have $\left(g_{\mathrm{PSF}}-i_{\mathrm{PSF}}\right)<1.153 \times\left(i_{\mathrm{PSF}}-K_{\mathrm{AB}}\right)-1.401$ to avoid the color locus of the stellar main sequence, and $\left(u_{\mathrm{PSF}}-g_{\mathrm{PSF}}\right)>0.4$ to exclude UV excess quasars (i.e., unreddened quasars at redshifts of about $z<2.15$ ). Here, the $K$-band magnitudes are on the Vega system, while the SDSS photometry is AB. The targets not included in Stripe 82 were effectively incorporated into the BONUS sample through the XDQSO selection (Bovy et al. 2011a, 2012) and this ancillary program was not continued for the remainder of the BOSS footprint. This ancillary program superseded the ancillary program "A Large Sample of Reddened Quasars," as the superior UKIDSS photometry could better target red, reddened, and high redshift quasars than 2MASS. In addition, the cut of $\left(u_{\mathrm{PSF}}-g_{\mathrm{PSF}}\right)>0.4$ better complemented the BOSS mission to target mostly higher redshift quasars.

\section{B.2. Full BOSS Survey}

The remaining BOSS ancillary science programs are distributed over some or all of the full 10,000 $\mathrm{deg}^{2}$ footprint and are selected from single-epoch imaging data. Observations will continue through the end of the survey, and additional programs will be added in the future. As with the Stripe 82 programs, we list these programs in order of increasing distance.

Very low mass stars and brown dwarfs. Very low mass stars and brown dwarfs (spectral types M8, M9 and L) are ideal tracers of the kinematic properties of the Milky Way thin disk. While the program "The Transient Universe Through Stripe 82" uses variability information over Stripe 82 to identify rare classes of stars, this program uses photometric selection over the much larger BOSS survey area to identify new low mass stars and brown dwarfs.

SDSS I and II yielded a wealth of spectroscopic data of these ultracool dwarfs (Schmidt et al. 2010c; West et al. 2011), but were limited by the small number of spectra observed for stars with spectral types later than M7. Additional observations of these objects are essential to understand the properties of magnetic activity in these ultracool dwarfs (extending the results of West et al. 2008). The data will also enable us to use kinematics to understand the distribution of ages, especially at the stellar/sub-stellar boundary. Finally, the sample will contain a class of $\mathrm{L}$ dwarfs that are peculiarly blue in the near-infrared but have typical L dwarf colors in SDSS $i-z$ (Schmidt et al. 2010b).

The program was divided into different densities in Stripe82 $\left(5 \mathrm{deg}^{-2}\right)$ and the rest of the BOSS footprint $\left(1 \mathrm{deg}^{-2}\right)$. In order to select the cleanest possible sample, we included 2MASS magnitudes as part of our selection criteria. Our Stripe 82 criteria were
1. $i_{\mathrm{PSF}}-z_{\mathrm{PSF}}>1.14$

2. $i_{\mathrm{PSF}}<21$

3. $i_{\mathrm{PSF}}-J>3.7$

4. $1.9<z_{\mathrm{PSF}}-J<4$.

The criteria for the rest of the BOSS footprint are

1. $i_{\mathrm{PSF}}-z_{\mathrm{PSF}}>1.44$

2. $i_{\mathrm{PSF}}<20.5$

3. $i_{\mathrm{PSF}}-J>3.7$

4. $1.9<z_{\mathrm{PSF}}-J<4$.

Here, the $J$-band photometry, unlike the SDSS photometry, is Vega-based.

The targets are divided into subsamples with different priorities. L dwarfs are both less common and fainter, so our priority was to detect $\mathrm{L}$ dwarfs over late-M dwarfs. Targets likely to be bright L dwarfs (BRIGHTERL; $i_{\mathrm{PSF}}<19.5$, $\left.i_{\mathrm{PSF}}-z_{\mathrm{PSF}}>1.14\right)$ are assigned first priority and fainter $\mathrm{L}$ dwarfs (FAINTERL; $i_{\mathrm{PSF}}>19.5, i_{\mathrm{PSF}}-z_{\mathrm{PSF}}>1.14$ ) are assigned second priority. The bright $\mathrm{M}$ dwarfs (BRIGHTERM; $\left.i_{\mathrm{PSF}}<19.5, i_{\mathrm{PSF}}-z_{\mathrm{PSF}}<1.14\right)$ are assigned third priority and FAINTERM dwarfs are assigned lowest priority.

Low-mass binary stars. Ultra-wide, low-mass binaries probe how dynamical interactions affect and shape the star formation process and the environment in which the processes occur. The binaries are also ideal coeval laboratories to constrain and calibrate properties of low-mass stars, as they were presumably born at the same time of the same material. The Sloan Low-mass Wide Pairs of Kinematically Equivalent Stars (SLoWPoKES; Dhital et al. 2010) catalog is the largest sample of wide, lowmass binaries. The objects in the catalog were identified from common proper motions and photometric distances but lacked information regarding radial velocities. The BOSS spectra will be used to measure radial velocities for the components and confirm their physical association. Targets for this program were selected in two ways: (1) SLoWPoKES systems of spectral type M0 or later, which had robust SDSS/USNO-B proper motions (Munn et al. 2004) and (2) systems of spectral type M4 or later, without proper motions. Both sets of targets were selected to have angular separations of $65^{\prime \prime}-180^{\prime \prime}$ to avoid fiber collision and to be brighter than $i_{\text {fib2 }}=20$ to achieve the critical $\mathrm{S} / \mathrm{N}$. The pairs from the second sub-sample are valuable for probing the lower-mass mid- and late-type $\mathrm{M}$ stars, which were underrepresented in SLoWPoKES. Although the lack of proper motion matching for these pairs makes them more susceptible to chance alignments, radial velocities from BOSS spectroscopy will confirm their common motions. From the combined sample, 500 pairs were randomly selected for targeting with BOSS, and both components will be observed. The spectra will be used to measure and calibrate the metallicity and mass-age-activity relationship for low-mass stars (e.g., Dhital et al. 2012), especially at the mid-late M spectral types. There was an error in correcting the positions of the target for their proper motions in the first year, affecting targets in plates numbered less than 3879 and between 3965-3987; few of these targets resulted in usable spectra.

White dwarfs and hot subdwarf stars. The SDSS multicolor imaging efficiently distinguishes hot white dwarf and subdwarf stars from the bulk of the stellar and quasar loci (Harris et al. 2003). Special target classes in SDSS produced the largest spectroscopic samples of white dwarfs (Kleinman et al. 2004; Eisenstein et al. 2006). However, much of SDSS white dwarf targeting required that the objects be unblended, which caused many brighter white dwarfs to be skipped (for a detailed 
discussion, see Section 5.6 of Eisenstein et al. 2006). This BOSS ancillary program relaxes this requirement and imposes color cuts to focus on warm and hot white dwarfs. Importantly, the BOSS spectral range extends further into the UV, allowing full coverage of the Balmer lines. We require targets to be point sources with good $u, g$, and $r$ photometry (following the clean point source selection from the DR7 documentation) and USNO counterparts. We restrict to regions inside the DR7 footprint with Galactic extinction of $A_{r}<0.5$ mag. Targets must satisfy $g<19.2,(u-r)<0.4,-1<(u-g)<0.3$, and $-1<(g-r)<0.5$, using extinction-corrected model magnitudes. Additionally, targets that do not have $u-r<-0.1$ and $g-r<-0.1$ must have USNO proper motions of more than $2^{\prime \prime}$ /century. Objects satisfying the selection criteria and not observed in previously in SDSS are denoted by the WHITEDWARF_NEW target flag, while those with SDSS spectra are assigned the WHITEDWARF_SDSS flag. Some of the latter objects are re-observed with BOSS in order to obtain the extended wavelength coverage. This color selection includes DA stars with temperatures above $\sim 14,000 \mathrm{~K}$ and helium atmosphere white dwarfs above $\sim 8000 \mathrm{~K}$, as well as many rarer classes of white dwarfs. Hot subdwarfs ( $\mathrm{sdB}$ and sdO) will be included as well. Many of these stars are excellent spectrophotometric standards and can be tested in comparison to the BOSS F-star calibration.

Distant halo giant stars. Rare giants in the outer halo of the Milky Way are selected using a targeting strategy tested in SEGUE2 (the SDSS-III counterpart to SEGUE; Yanny et al. 2009; C. M. Rockosi et al. 2013, in preparation). Observations of the new BOSS targets are expected to increase the number of known halo stars beyond $60 \mathrm{kpc}$ by a factor of 10 . The full sample RED_KG will enable measurements of substructure of the Milky Way in position-velocity phase space, a signature of the hierarchical assembly of the stellar halo. The data will thus provide tests of $\Lambda \mathrm{CDM}$ predictions for galaxy assembly in a part of the Galaxy expected to be almost entirely composed of debris from recent accretions (Xue et al. 2011). The sample will also enable dynamical mass estimates for the Milky Way out to $150 \mathrm{kpc}$. Objects with $17<g_{\text {fib2 }}<19.5$ are selected from a color box, with $\left(u_{\mathrm{PSF}}-g_{\mathrm{PSF}}, g_{\mathrm{PSF}}-r_{\mathrm{PSF}}\right)$ corners of $(0.8,2.35),(0.8,2.65),(1.4,3.0)$, and $(1.4,3.9)$. This selection identifies stars near the tip of the red giant branch in the region of the $u-g / g-r$ diagram where giants separate from the locus of foreground dwarfs (Yanny et al. 2009). In addition,

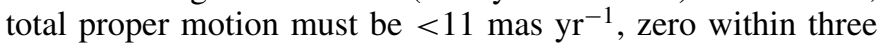
standard deviations of the proper motion errors, to reject nearby stars. The 3 " fiber magnitude must be $i>16.5$ as an additional mechanism to reject bright targets. A smaller sample of targets is found on the commissioning plates. Denoted RVTEST, this sample targets stars previously observed spectroscopically as a test of the reproducibility of velocity measurements.

Bright galaxies. Bright galaxies were commonly missed in the original SDSS spectroscopic survey due to fiber collisions, bright limits (objects with model magnitudes $r>15, g>15$, or $i>14.5$ were excluded), and errors in the deblending of overlapping images (Strauss et al. 2002). Approximately 10\% of the brightest galaxies were not spectroscopically observed (Fukugita et al. 2007). To improve the completeness of this spectroscopic sample, objects were chosen with Petrosian radius $>1^{\prime \prime}$ in $r$ to reject stars, with no saturated pixels, and with extinction-corrected, Petrosian (Petrosian 1976; Strauss et al. 2002) $r$ magnitude between 10 and 16. Targets were also required to have an extinction-corrected Petrosian magnitude $i<20$ and $z<20$ to exclude misidentified satellite tracks that would not show up in the other bands. Galaxies without spectra ( $\sim 24,000$ from the original list of $\sim 93,000)$ where then visually vetted to remove foreground stars that remained in the sample, detector artifacts (e.g., internal reflections) that were misidentified, and other sources of confusion. In cases where a foreground star was misidentified as the galaxy center, the target position was moved to the correct position. In cases of merging galaxies, we visually identified multiple targets corresponding to the centers of each galaxy. The list was cross-correlated with the Third Reference Catalog of Bright Galaxies (RC3 de Vaucouleurs et al. 1991; Corwin et al. 1994), and any targets that did not appear in the original SDSS spectroscopic survey were added to the target list $(0.05 \%$ of the final list). Finally, targets within $2^{\prime \prime}$ of a star that appears in the Tycho-2 Catalog (Høg et al. 2000) were removed. The final sample includes 8637 galaxies over the BOSS footprint.

High energy blazars and optical counterparts of gamma-ray sources. We targeted candidate optical counterparts of sources detected (or likely to be detected) by NASA's Fermi Gammaray Space Telescope (Atwood et al. 2009), with the goal to spectroscopically confirm and provide redshifts for candidate gamma-ray blazars, with model magnitude $m<21$ in any of the three bandpasses $g, r$, or $i$. We also require targets to have $3^{\prime \prime}$ fiber magnitudes $m>16.5$ to minimize impact of fiber crosstalk. Ranked in approximate order of priority, fibers are assigned to targets from the following subprograms.

1. BLAZGXR: about 300 blazar candidates are assigned at highest priority to DR7 optical sources within Fermi gamma-ray error ellipses. Targets must also lie within the $<1^{\prime}$ radius error circle for X-ray sources in the ROSAT AllSky Survey (RASS; Voges et al. 1999, 2000) and within $2^{\prime \prime}$ of a FIRST (Becker et al. 1995) radio source.

2. BLAZGRFLAT: about 175 blazar candidates detected with Fermi and the Combined Radio All-Sky Targeted Eight GHz Survey (CRATES; Healey et al. 2007). Objects from the DR7 catalog within 2" of a CRATES radio source and within a Fermi error ellipse were targeted.

3. BLAZGXQSO: 95 further candidate X-ray and gamma-ray emitting quasars/blazars, including photometric quasar/ blazar candidates (Richards et al. 2009), as well as confirmed DR7 quasars/blazars (Schneider et al. 2010) revisited to assess optical spectral variability. Targets are selected that lie within $<1^{\prime}$ of a RASS X-ray source and within Fermi error ellipses.

4. BLAZGRQSO: 185 candidate radio and gamma-ray emitting quasars/blazars, including both photometric candidates (Richards et al. 2009), and DR7 confirmations (Schneider et al. 2010) revisited to assess optical spectral variability. Targets are selected that lie within $2^{\prime \prime}$ of a FIRST radio source and within Fermi error ellipses.

5. BLAZGX: 75 targets that are candidate high-energy counterparts but which lack typical (e.g., radio emission, unusual optical color, etc.) blazar properties were targeted to probe unknown classes of gamma ray sources. The optically brightest objects from DR7 within the Fermi error ellipses and within $1^{\prime}$ of a RASS X-ray source were preferentially targeted.

6. BLAZXR: 1100 targets are selected that may plausibly emerge as Fermi sources, but are still below the detection limits in the early Fermi source catalogs. The approach is similar to the "ROSAT_A" target selection scheme described in Anderson et al. (2003) and the "pre-selection" 
approach of Healey et al. (2008) that provided many of the gamma-ray counterpart associations reported in the first Fermi catalogs (Abdo et al. 2010b, 2010a). Targets are chosen from the DR7 photometry catalog with radio coincidence (within $2^{\prime \prime}$ of a FIRST source) and X-ray coincidence ( $<1^{\prime}$ of a RASS source). This sample overlaps heavily with the BONUS quasar sample, but includes quasars at lower redshift.

In addition, there were 10 miscellaneous candidate blazar spectra taken in an early trial of this program. These targets were assigned subcategory names using the following flags: BLAZGVAR, BLAZR, and BLAZXRSAM.

An $X$-ray view of star formation and accretion in normal galaxies. The extended wavelength coverage and improved throughput of BOSS relative to SDSS enable studies of the relationship between star formation and black hole accretion in galaxies using the key diagnostic $\mathrm{H} \alpha / \mathrm{N}[\mathrm{II}]$ to $z \sim 0.49$. For this study, a target list was derived from the matched Chandra Source Catalog (CSC; version 1; Evans et al. 2010) and SDSS DR7 photometric catalogs. High quality matches underwent visual inspection in X-ray and optical imaging and were required to have positional matches between the SDSS DR7 catalog and the CSC as defined by the Rots et al. (2009) matching method that incorporates positions, positional errors, and sky coverage. Matches with Bayesian probability $<0.5$ suffer from a larger number of multiple matches and are discarded. In addition, targets were required to have model magnitude $16.5<$ $r<20.75$ and Chandra off-axis angles $\theta<10^{\prime}$. Objects with existing SDSS spectroscopy, proper motions from Munn et al. $(2004,2008)$ exceeding 11 mas $\mathrm{yr}^{-1}$ (selection criteria are described further in Haggard et al. 2010), or poor-quality $\mathrm{X}$-ray measurements are removed from the sample. Because only sources with Chandra coverage were included, the target have a non-uniform distribution over the DR7 footprint. To avoid overlap between the targets selected for this program and the program "Remarkable X-ray Source Populations" described below, the target lists were cross-correlated (using a $2^{\prime \prime}$ match radius) and 754 duplicates were removed from this program designation.

Remarkable X-ray source populations. This program targets remarkable serendipitous X-ray sources from the Second XMM-Newton Serendipitous Source Catalog (2XMMi; Watson et al. 2009) and the CSC (Evans et al. 2010) that do not already have available identification spectra. Source types of primary interest include AGN (often obscured), X-ray binaries, magnetic cataclysmic variables, and strongly flaring stars.

$X M M-N e w t o n$ sources were required to lie within $14^{\prime}$ of the pointing center of an XMM-Newton observation, to have a 2XMMi detection likelihood greater than 12 as defined in Watson et al. (2009), to have a statistically significant match to an SDSS photometric counterpart, and not to have any 2XMMi problem flags set. Similarly, Chandra sources were required to have an SDSS counterpart and not to have any indication of Chandra source confusion. All XMM-Newton and Chandra sources having Galactic latitudes of $|b|<20^{\circ}$ were furthermore removed to emphasize extragalactic sources.

The four XMM-Newton source types for this program were all selected using SDSS model magnitudes and are determined as follows:

1. XMMHR: $1030 X M M-N e w t o n$ sources were selected that have unusual 2XMMi hardness ratios in the HR2-HR3 plane. These sources were also required to have $i<20.5$ (to ensure reasonable BOSS spectral quality) and to have
$2-12 \mathrm{keV} \mathrm{X}$-ray to $i$-band flux ratios greater than 0.03 (to minimize stellar contamination). Optical fluxes are defined as $f_{v} \Delta v$, where $\Delta v$ is the width of the bandpass.

2. XMMBRIGHT: 826 XMM-Newton sources were selected that have bright $2-12 \mathrm{keV}$ fluxes (brighter than $5 \times$ $10^{-14} \mathrm{erg} \mathrm{cm}^{-2} \mathrm{~s}^{-1}$ ). These sources were also required to have $i<20.0$.

3. XMMRED: 627 optically red XMM-Newton sources were selected that have SDSS colors of $g-i>1.0$. These sources were also required to have $i<19.3$ and to have $2-12 \mathrm{keV} X$-ray to $i$-band flux ratios greater than 0.03 .

4. XMMGRIZ: 149 XMM-Newton sources were selected that have "outlier" SDSS colors. Specifically, we selected SDSS point-source counterparts that have $g-r>1.2$ or $r-i>1.0$ or $i-z>1.4$. These sources were also required to have $i=18-21.3$ and to have $0.5-2 \mathrm{keV}$ X-ray to $i$-band flux ratios greater than 0.1 .

There is some overlap among these XMM-Newton sourceselection approaches (e.g., a bright XMM-Newton source might also have optically red colors). At each selection step, we removed sources already selected in previous XMM-Newton selection steps (following the ordering above). There are 2632 selected XMM-Newton sources in total.

As with the XMM-Newton sources, Chandra source types were all selected using SDSS model magnitudes and are determined as follows:

1. CXOBRIGHT: 387 Chandra sources were selected that have bright $2-8 \mathrm{keV}$ fluxes (brighter than $5 \times$ $10^{-14}$ erg $\mathrm{cm}^{-2} \mathrm{~s}^{-1}$ ). These sources were also required to have $i<20.0$.

2. CXORED: 635 optically red Chandra sources were selected that have SDSS colors of $g-i>1.0$. These sources were also required to have $i<19.3$ and to have $2-8 \mathrm{keV}$ $\mathrm{X}$-ray to $i$-band flux ratios greater than 0.03 .

3. CXOGRIZ: 66 Chandra sources were selected that have "outlier" SDSS colors. Specifically, we selected SDSS point-source counterparts that have $g-r>1.2$ or $r-i>$ 1.0 or $i-z>1.4$. These sources were also required to have $i=18-21.3$ and to have $0.5-2 \mathrm{keV} \mathrm{X}$-ray to $i$-band flux ratios greater than 0.1 .

Again, there is some overlap among these Chandra sourceselection approaches, and again at each step we removed sources already selected in previous Chandra selection steps (following the ordering above). There are 1088 selected Chandra sources in total. We furthermore removed selected Chandra sources that were also selected XMM-Newton sources; this reduced the number of selected Chandra sources to 952.

Star-Forming Radio Galaxies. Joint analysis of SDSS, FIRST, and the NRAO VLA Sky Survey (NVSS; Condon et al. 1998) has shown that low redshift radio AGNs play an essential role in regulating the growth of massive galaxies (e.g., Best et al. 2005, 2007). However, much less is known about the detailed interplay of gas cooling and radio feedback in more luminous radio galaxies at higher redshifts. Current samples are incomplete, in particular for radio galaxies with significant on-going star formation. This ancillary program selects radio galaxies with blue colors at $z>0.3$ that would otherwise be missed from the LOWZ and CMASS samples. Galaxy targets were selected from DR7 according to the following criteria: (1) extended morphology in SDSS photometry; (2) clean ugriz model photometry and $17<i<19.9$; (3) $i_{\text {fib } 2}<21.7$; and (4) $[(g-r)>1.45]$ or $[(u-g)<1.14 *(g-r)]$, where 
photometry is determined from model magnitudes. The later criterion is designed to color-select objects at $z>0.3$. We cross-matched this sample with the FIRST catalog (2008 July version) and selected all objects within $3^{\prime \prime}$ of FIRST sources with fluxes $>3.5 \mathrm{mJy}$. Most targets were within $1^{\prime \prime} .5$. Finally, we rejected objects spectroscopically observed by SDSS-I/II, and objects meeting the target selection criterion for the galaxy samples. In total there were 4610 targets; we randomly sampled these to produce a final list of 4170 ancillary targets.

Galaxies near SDSS quasar sight lines. Obtaining accurate redshifts of galaxies projected near the lines of sight to quasars with existing SDSS spectra allows a study of the properties of galaxies that are associated with intervening quasar absorption systems. BOSS enables a study at small galaxy-quasar separations that could not be done with SDSS due to fiber collisions. Galaxy targets are selected by a $u g r$ color cut to lie in a redshift range $(z>0.35)$ where $\mathrm{Mg}$ II at $2800 \AA$ is detectable in SDSS spectra. The sample of spectroscopically confirmed quasars was selected with model magnitude $g<19.2$ and redshift $0.7<z<2.1$ from the SDSS DR7 quasar catalog (Schneider et al. 2010). Galaxies were chosen that lie between 0 '006 and $1^{\prime}$ of a quasar with spectroscopy, with model magnitudes $17.5<i<19.9, A_{g}<0.3$, and $(g-r)>1.65$ or $(u-g)<1.14 \times(g-r)-0.4$ to select objects with $z>0.35$. The sample is weighted to have similar numbers of galaxies at separations $b<0$.'5 and $0.5<b<1^{\prime}$. A sample of approximately 3000 galaxies will help determine whether absorption is correlated with galaxy type, the covering fraction of absorption as a function of radius, and the size of velocity offsets.

Luminous blue galaxies at $0.7<z<1.7$. Studies from the second Deep Extragalactic Evolutionary Probe (DEEP2; Davis et al. 2003) reveal that the most luminous, star-forming blue galaxies at $z \sim 1$ appear to be a population that evolves into massive red galaxies at lower redshifts (Cooper et al. 2008). Sampling 2000 color-selected galaxies in Stripe 82 and from the CFHTLS Wide fields (W1, W3, and W4) allows a measure of the clustering of the rarest, most luminous of these blue galaxies on large scales. Such a measurement has not previously been conducted, as prior galaxy evolution-motivated surveys have a limited field of view and mostly target fainter galaxies.

The galaxy targets were color-selected based on the CFHTLS photometric-redshift catalog (Coupon et al. 2009). Different color selections were explored using either the $\left(u_{\mathrm{PSF}}-g_{\mathrm{PSF}}\right.$, $\left.g_{\mathrm{PSF}}-r_{\mathrm{PSF}}\right)$ color-color diagram down to $g_{\mathrm{PSF}}<22.5$ or the $\left(g_{\mathrm{PSF}}-r_{\mathrm{PSF}}, r_{\mathrm{PSF}}-i_{\mathrm{PSF}}\right)$ color-color diagram down to $i_{\mathrm{PSF}}<21.3$. Detailed description of the color selection and redshift measurement is in Comparat et al. (2012). Using this dataset, photometric redshifts can be re-calibrated in the CFHTLS W3 field, thereby reducing biases in redshift estimates at $z>1$. Measurement of the galaxy bias of these luminous blue galaxies will be presented in J. Comparat et al (2013, in preparation). This dataset has been important in motivating the "extended-BOSS" project (PI, J.-P. Kneib), a survey that is proposed to begin in Fall 2014 as part of the successor to SDSS-III. The data will also be used to improve the targeting strategy of future projects such as BigBOSS (Schlegel et al. 2011).

Broad absorption line (BAL) Quasar Variability Survey. Thousands of BAL quasars were discovered in the SDSS-I and II (e.g., Gibson et al. 2009). In some cases, repeat spectroscopy showed variable absorption, providing clues to the nature of the BAL phenomenon (e.g., Lundgren et al. 2007; Gibson et al.
2008 , 2010). Returning with BOSS to obtain repeat spectra on a much larger sample of these quasars allows a large-scale study of BAL variability on multi-year timescales in the rest frame. The resulting data provide insight into the dynamics, structure, and energetics of quasar winds. First results from this ancillary project are presented in Filiz Ak et al. (2012).

The targets for this ancillary project were selected before the decision was made to re-target known quasars at $z>2.15$ (see Section 2.4), and thus there is some overlap between these two samples. However, this ancillary project also provides many unique targets at $z<2.15$. The main sample of BAL quasars chosen for study contains 2005 objects assigned the ancillary target flag VARBAL; this sample is about two orders of magnitude larger than those used previously to investigate BAL variability on multi-year timescales. These 2005 objects were selected to be optically bright $\left(i_{\mathrm{PSF}}<19.28\right.$ with no correction for extinction) and to have at least moderately strong absorption in one of their BAL troughs (with a "balnicity index" of $\mathrm{BI}_{0}>100 \mathrm{~km} \mathrm{~s}^{-1}$ as measured by Gibson et al. 2009). In addition, only quasars that are in redshift ranges such that strong BAL transitions are fully covered by the SDSS-I, SDSS-II and BOSS spectra (from outflow velocities of $0-25000 \mathrm{~km} \mathrm{~s}^{-1}$ ) were included; see Section 4 of Gibson et al. (2009) for further explanation. The corresponding redshift ranges are $1.96<z<5.55$ for Si IV BALs, $1.68<z<4.93$ for C IV BALs, $1.23<z<3.93$ for Al III BALs, and $0.48<z<2.28$ for Mg II BALs. Finally, for those objects in the Gibson et al. (2009) catalog that have measurements of the $\mathrm{S} / \mathrm{N}$ at rest-frame $1700 \AA\left(\mathrm{SN}_{1700}\right)$, we require that $\mathrm{SN}_{1700}>6$; this criterion ensures that high-quality SDSS/SDSS-II spectra are available for these targets.

In addition to the primary VARBAL sample objects described above, the BAL quasar variability survey also targets 102 additional BAL quasars selected with other approaches. These targets may violate one or more of the selection criteria utilized for the VARBAL targets, but they have been identified as worthy of new observations nonetheless. The relevant source types for these additional BAL quasars are the following.

1. LBQSBAL and FBQSBAL are BAL quasars identified in the Large Bright Quasar Survey (LBQS; e.g., Hewett et al. 1995) and the FIRST Bright Quasar Survey (FBQS; e.g., White et al. 2000), respectively. They thus have LBQS or FBQS spectra predating the SDSS-I and II spectra by up to a decade or more.

2. OTBAL (Overlapping-Trough BAL quasars) are BAL quasars with nearly complete absorption at wavelengths shortward of $\mathrm{Mg}$ II in one epoch and which in one case have already shown extreme variability (e.g., Hall et al. 2002).

3. PREVBAL are BAL quasars observed more than once by SDSS-I and II. They thus already possess more than one observation epoch for comparison to BOSS spectra.

4. ODDBAL are BAL quasars selected to have various unusual properties (e.g., Hall et al. 2002). For these objects, variability (or the lack thereof) between SDSS-I and II and BOSS may help to unravel the processes responsible for their unusual spectra.

Variable quasar narrow-line absorption. Quasar absorption lines are plentiful in SDSS I and II and have been documented in a catalog of all lines and systems (QSOALS; York et al. 2005). This catalog (D. G. York et al. 2013, in preparation), now updated through DR7, contains 60,000 uniformly detected 
quasar absorption line systems in which two or more transitions from common metal absorption lines (e.g., $\mathrm{Mg}$ II, Fe II, C IV) are identified at the same redshift. This dataset has been used to study the statistics of quasar absorption lines (York et al. 2006) and to confirm correlations with quasars (Wild et al. 2007) as well as foreground galaxies projected along the line of sight (Lundgren et al. 2009).

It has been shown that smaller equivalent width BALs are more prone to variation on short timescales (e.g., Barlow 1994; Lundgren et al. 2007). A large survey of variability in narrow absorption lines (NALs) is therefore required in order to examine if this trend applies across a larger range in equivalent widths. Complementary to the ancillary BAL quasar variability study described above, this program seeks to compile the largest dataset of multi-epoch observations of quasar sight lines with known narrow absorption along the line of sight. Detections of variability in NAL systems hold great promise for identifying high-velocity intrinsic quasar absorption and miniBAL emergence and for providing limits on the sizes of cold gas clouds in the extended haloes of luminous galaxies in the foreground.

The targets of this program include quasars with $16.5<$ $i_{\text {fib2 }}<17.9$ and redshift $0.7<z<2.2$ from the DR7 quasar catalog (Schneider et al. 2010), which would otherwise be ignored by the primary BOSS target selection. Sight lines with known BALs (Gibson et al. 2008) are ignored, as this parameter space is being covered by the separate BOSS BAL variability program. Each of the sight lines targeted in this program contains a NAL system detected at $>4 \sigma$ (including multiple unambiguous transitions of $\mathrm{Mg}$ II, C IV, or both), which have been identified in York et al. (2005). However, this program is not limited only to cases in which previously identified NALs disappear in later epochs, since NALs should have the same probability of emerging along these lines of sight with and without identified NALs. In total, this program targets about 3000 quasars with a target density of $\sim 0.35 \mathrm{deg}^{-2}$.

As one of the science goals of this program is to determine the extent of variable NALs in velocity space relative to the quasar, the target list includes sight lines with NALs over a wide range in velocity. The following sub-groups allow for the identification of quasars and absorbers with particular characteristics.

1. QSO_RADIO_AAL: radio-loud with 1 associated absorption system (AAL; $v \leqslant 5000 \mathrm{~km} \mathrm{~s}^{-1}$ in the quasar rest frame).

2. QSO_RADIO_IAL: radio-loud with 1 intervening absorption system (IAL: $v>5000 \mathrm{~km} \mathrm{~s}^{-1}$ in the quasar rest frame).

3. QSO_AAL: radio-quiet source with 1 AAL

4. QSO_IAL: radio-quiet source with 1 IAL

5. QSO_RADIO: radio-loud source with multiple AALs and/ or IALs

6. QSO_AALs: radio-quiet source with multiple AAL and/or IALs

7. QSO_noAALs: radio-quiet source with no AALs and multiple IALs

Double-lobed radio quasars. Objects identified as optical point sources near the midpoint of pairs of FIRST radio sources are observed as potential double-lobed radio quasars. Such quasars are important for studying quasar evolution and interactions of radio jets with their local environment. Candidates are selected by identifying FIRST pairs with a separation less than $60^{\prime \prime}$ and no SDSS optical counterpart within $2^{\prime \prime}$ of either source. SDSS point sources located within a search radius that ranges between $2^{\prime \prime}$ and 5.3 (depending on the separation distance of the FIRST pair) from the midpoint are targeted. FIRST pairs with a flux ratio $>10$ are rejected because true double-lobed sources are unlikely to have a high ratio of lobe-lobe flux density. The final catalog includes objects not spectroscopically observed with SDSS, not targeted in the main BOSS sample, and with $17.8<i_{\mathrm{PSF}}<21.6$ (Galactic extinction-corrected).

High redshift quasars. High-redshift quasars trace the evolution of early generations of supermassive black holes, provide tests for models of quasar formation and AGN evolution, and probe evolution in the intergalactic medium (IGM). However, the BOSS quasar survey (Ross et al. 2012b) selects objects only to $z \sim 3.5$. Light emitted by high redshift quasars at wavelengths shorter than Ly $\alpha$ is absorbed by the IGM, meaning that for redshifts $z>5.7$, quasars are detected in only the $z$ band, the reddest filter in the SDSS imaging survey. We use areas with overlap imaging, thereby reducing contamination from cosmic rays and improving the photometry, to select high redshift quasar candidates in three redshift ranges to fainter magnitudes than in the SDSS survey.

For the main survey, PSF magnitudes for objects with multiple detections are extracted from the Neighbors table in the DR7 Catalog Archive Server, and the detections are co-added in each band. Target selection is performed on the co-added photometry. For objects within Stripe 82, PSF magnitudes were extracted from the co-added image catalogs described in Annis et al. (2011). These catalogs combine roughly 20 epochs instead of just two, and permit selection of objects at fainter magnitudes. The first part of the program targets objects with similar color cuts imposed in the SDSS quasar survey (Richards et al. 2002). The SDSS quasar target selection defined two inclusion regions in $g r i$ and $r i z$ color space for targeting $z>3.6$ and $z>4.5$ quasars, respectively (Richards et al. 2002). The first two high- $z$ quasar ancillary programs are straightforward extensions of these color selection criteria to fainter magnitudes, with limits of $i_{\mathrm{PSF}}<21.3$ in the main survey regions and $i_{\mathrm{PSF}}<21.5$ (compared to $i_{\mathrm{PSF}}<20.2$ for SDSS). The ancillary target flag QSO_GRI is assigned to objects meeting the gri color criteria, and the flag QSO_RIZ to those meeting the riz criteria. In both cases, the primary color cut follows a diagonal line in the respective color plane (e.g., $r-i<A(g-r)+B$ for the $r i z$ cut), and the intercept of this line is shifted slightly upwards for brighter objects. This approach allows objects with similar brightness to SDSS quasars to be selected with more relaxed color criteria. This sample will provide a probe of the quasar luminosity function at high redshift and improve small-scale clustering measurements. A final class of targets (QSO_HIZ) in this program are candidates for quasars with redshifts between 5.6 and 6.5. At these redshifts, quasars have extremely red $i-z$ colors; this program targets all objects with $(i-z)>1.6$ and $z<20.8$ and that have no detections in the other SDSS bands.

High-redshift quasars from SDSS and UKIDSS. The final ancillary program described here targets high redshift quasar candidates through a combination of color cuts combining SDSS ugriz PSF photometry and UKIDSS $Y J H K$ aperture photometry ( $1^{\prime \prime}$ radius apertures). The addition of IR photometry from UKIDSS provides leverage to separate quasars at $z \sim 5.5$ from the red end of the stellar locus-indeed, the SDSS quasar survey was limited to $z<5.4$ by the strong overlap in ugriz colors of higher redshift quasars with red stars. Quasar candidates were selected by matching stellar objects from the 
SDSS DR7 UKIDSS DR3 databases. The initial sample is drawn from SDSS with the cuts $r-i>1.4, i-z>0.5$, and $z<20.2$. Likely stars are rejected with the criteria $(H-K)_{\text {Vega }}<0.53$ or $(J-K)_{\text {Vega }}<1.3(Y-J)_{\text {Vega }}+0.32$, taking advantage of the fact that quasars are redder than $M$ stars at longer wavelengths and bluer at shorter wavelengths. The remaining candidates are then prioritized based on $i z Y J K$ colors. For Stripe 82, the color criteria used for prioritizing targets were slightly relaxed, owing to the co-added ugriz photometry available from Annis et al. (2011), which greatly reduced the initial stellar contamination from the riz selection. Objects selected with the relaxed criteria on Stripe 82 are given the HIZQSO82 target bit.

\section{REFERENCES}

Abazajian, K., Adelman-McCarthy, J. K., Agüeros, M. A., et al. 2004, AJ, 128,502

Abazajian, K. N., Adelman-McCarthy, J. K., Agüeros, M. A., et al. 2009, ApJS, 182,543

Abdo, A. A., Ackermann, M., Ajello, M., et al. 2010a, ApJ, 715, 429

Abdo, A. A., Ackermann, M., Ajello, M., et al. 2010b, ApJS, 188, 405

Adelman-McCarthy, J. K., Agüeros, M. A., Allam, S. S., et al. 2008, ApJS, 175,297

Adelman-McCarthy, J. K., Agüeros, M. A., Allam, S. S., et al. 2006, ApJS, 162,38

Ahn, C. P., Alexandroff, R., Allende, P. C., et al. 2012, arXiv:1207.7137

Aihara, H., Allende, P. C., An, D., et al. 2011a, ApJS, 193, 29

Aihara, H., Allende Prieto, C., An, D., et al. 2011b, ApJS, 195, 26

Albrecht, A., Bernstein, G., Cahn, R., et al. 2006, arXiv:astro-ph/0609591

Anderson, L., Aubourg, E., Bailey, S., et al. 2012, arXiv:1203:6594

Anderson, S. F., Becker, A. C., Haggard, D., et al. 2008, AJ, 135, 2108

Anderson, S. F., Voges, W., Margon, B., et al. 2003, AJ, 126, 2209

Annis, J., Soares-Santos, M., Strauss, M. A., et al. 2011, arXiv:1111.6619

Atwood, W. B., Abdo, A. A., Ackermann, M., et al. 2009, ApJ, 697, 1071

Auger, M. W., Treu, T., Bolton, A. S., et al. 2009, ApJ, 705, 1099

Barlow, T. A. 1994, PASP, 106, 548

Becker, A. C., Agol, E., Silvestri, N. M., et al. 2008, MNRAS, 386, 416

Becker, A. C., Bochanski, J. J., Hawley, S. L., et al. 2011, ApJ, 731, 17

Becker, R. H., White, R. L., \& Helfand, D. J. 1995, ApJ, 450, 559

Best, P. N., Kauffmann, G., Heckman, T. M., et al. 2005, MNRAS, 362, 25

Best, P. N., von der Linden, A., Kauffmann, G., Heckman, T. M., \& Kaiser, C. R. 2007, MNRAS, 379, 894

Beutler, F., Blake, C., Colless, M., et al. 2011, MNRAS, 416, 3017

Bhatti, W. A., Richmond, M. W., Ford, H. C., \& Petro, L. D. 2010, ApJS, 186,233

Blake, C., Davis, T., Poole, G. B., et al. 2011a, MNRAS, 415, 2892

Blake, C., \& Glazebrook, K. 2003, ApJ, 594, 665

Blake, C., Kazin, E. A., Beutler, F., et al. 2011b, MNRAS, 418, 1707

Blake, C. H., Torres, G., Bloom, J. S., \& Gaudi, B. S. 2008, ApJ, 684, 635

Blanton, M. R., Lin, H., Lupton, R. H., et al. 2003, AJ, 125, 2276

Bolton, A. S., Brownstein, J. R., Kochanek, C. S., et al. 2012a, ApJ, 757, 82

Bolton, A. S., Burles, S., Koopmans, L. V. E., Treu, T., \& Moustakas, L. A. 2006, ApJ, 638, 703

Bolton, A. S., \& Schlegel, D. J. 2010, PASP, 122, 248

Bolton, A. S., Schlegel, D. J., Aubourg, É., et al. 2012b, AJ, 144, 144

Bolton, A. S., Treu, T., Koopmans, L. V. E., et al. 2008, ApJ, 684, 248

Bovy, J., Hennawi, J. F., Hogg, D. W., et al. 2011a, ApJ, 729, 141

Bovy, J., Hogg, D. W., \& Roweis, S. T. 2011b, AnApS, 5, 1657

Bovy, J., Myers, A. D., Hennawi, J. F., et al. 2012, ApJ, 749, 41

Bramich, D. M., Vidrih, S., Wyrzykowski, L., et al. 2008, MNRAS, 386, 887

Brandt, T. D., Tojeiro, R., Aubourg, É., et al. 2010, AJ, 140, 804

Brownstein, J. R., Bolton, A. S., Schlegel, D. J., et al. 2012, ApJ, 744, 41

Butler, N. R., \& Bloom, J. S. 2011, AJ, 141, 93

Campbell, H., D’Andrea, C. B., Nichol, R. C., et al. 2012, ApJ, submitted (arXiv:1211:4480)

Cannon, R., Drinkwater, M., Edge, A., et al. 2006, MNRAS, 372, 425

Cardelli, J. A., Clayton, G. C., \& Mathis, J. S. 1989, ApJ, 345, 245

Chen, Y.-M., Kauffmann, G., Tremonti, C. A., et al. 2012, MNRAS, 421, 314

Cole, S., Percival, W. J., Peacock, J. A., et al. 2005, MNRAS, 362, 505

Colless, M., Dalton, G., Maddox, S., et al. 2001, MNRAS, 328, 1039

Comparat, J., Kneib, J.-P., Escoffier, S., et al. 2012, MNRAS, in press (arXiv:1207.4321)

Condon, J. J., Cotton, W. D., Greisen, E. W., et al. 1998, AJ, 115, 1693

Conley, A., Guy, J., Sullivan, M., et al. 2011, ApJS, 192, 1
Cooper, M. C., Newman, J. A., Weiner, B. J., et al. 2008, MNRAS, 383, 1058 Corwin, H. G., Jr., Buta, R. J., \& de Vaucouleurs, G. 1994, AJ, 108, 2128 Coupon, J., Ilbert, O., Kilbinger, M., et al. 2009, A\&A, 500, 981

Covey, K. R., Ivezić, Ž., Schlegel, D., et al. 2007, AJ, 134, 2398

Croft, R. A. C., Weinberg, D. H., Bolte, M., et al. 2002, ApJ, 581, 20

Croft, R. A. C., Weinberg, D. H., Katz, N., \& Hernquist, L. 1998, ApJ, 495, 44 Croft, R. A. C., Weinberg, D. H., Pettini, M., Hernquist, L., \& Katz, N. 1999, ApJ, 520, 1

D’Andrea, C. B., Gupta, R. R., Sako, M., et al. 2011, ApJ, 743, 172

Davis, M., Faber, S. M., Newman, J., et al. 2003, Proc. SPIE, 4834, 161

de Vaucouleurs, G. 1948, AnAp, 11, 247

de Vaucouleurs, G., de Vaucouleurs, A., Corwin, H. G., Jr., et al. 1991, Sky Telesc., 82, 621

Dhital, S., West, A. A., Stassun, K. G., \& Bochanski, J. J. 2010, AJ, 139, 2566

Dhital, S., West, A. A., Stassun, K. G., et al. 2012, AJ, 143, 67

Drinkwater, M. J., Jurek, R. J., Blake, C., et al. 2010, MNRAS, 401, 1429

Driver, S. P., Hill, D. T., Kelvin, L. S., et al. 2011, MNRAS, 413, 971

Driver, S. P., Norberg, P., Baldry, I. K., et al. 2009, A\&G, 50, 5.12

Eisenstein, D. J., Annis, J., Gunn, J. E., et al. 2001, AJ, 122, 2267

Eisenstein, D. J., Hu, W., \& Tegmark, M. 1998, ApJ, 504, L57

Eisenstein, D. J., Liebert, J., Harris, H. C., et al. 2006, ApJS, 167, 40

Eisenstein, D. J., Seo, H.-J., Sirko, E., \& Spergel, D. N. 2007, ApJ, 664, 675

Eisenstein, D. J., Weinberg, D. H., Agol, E., et al. 2011, AJ, 142, 72

Eisenstein, D. J., Zehavi, I., Hogg, D. W., et al. 2005, ApJ, 633, 560

Evans, I. N., Primini, F. A., Glotfelty, K. J., et al. 2010, ApJS, 189, 37

Falco, E. E., Kurtz, M. J., Geller, M. J., et al. 1999, PASP, 111, 438

Fan, X. 1999, AJ, 117, 2528

Fan, X., Strauss, M. A., Schneider, D. P., et al. 1999, AJ, 118, 1

Filiz Ak, N., Brandt, W. N., Hall, P. B., et al. 2012, ApJ, 757, 114

Font-Ribera, A., McDonald, P., \& Miralda-Escudé, J. 2012, JCAP, JCAP01(2012)001

Freeman, K. C. 1970, ApJ, 160, 811

Frieman, J. A., Bassett, B., Becker, A., et al. 2008, AJ, 135, 338

Fukugita, M., Ichikawa, T., Gunn, J. E., et al. 1996, AJ, 111, 1748

Fukugita, M., Nakamura, O., Okamura, S., et al. 2007, AJ, 134, 579

Ge, J., Lee, B., de Lee, N., et al. 2009, Proc. SPIE, 7440, 18

Geach, J. E., Murphy, D. N. A., \& Bower, R. G. 2011, MNRAS, 413, 3059

Gibson, R. R., Brandt, W. N., Gallagher, S. C., Hewett, P. C., \& Schneider, D. P. 2010, ApJ, 713, 220

Gibson, R. R., Brandt, W. N., Schneider, D. P., \& Gallagher, S. C. 2008, ApJ, 675,985

Gibson, R. R., Jiang, L., Brandt, W. N., et al. 2009, ApJ, 692, 758

Goranova, Y., Hudelot, P., Magnard, F., et al. 2009, The CFHTLS T0006

Release (http://terapix.iap.fr/cplt/T0006-doc.pdf)

Gunn, J. E., Carr, M., Rockosi, C., et al. 1998, AJ, 116, 3040

Gunn, J. E., Siegmund, W. A., Mannery, E. J., et al. 2006, AJ, 131, 2332

Haggard, D., Green, P. J., Anderson, S. F., et al. 2010, ApJ, 723, 1447

Hall, P. B., Anderson, S. F., Strauss, M. A., et al. 2002, ApJS, 141, 267

Hand, N., Addison, G. E., Aubourg, E., et al. 2012, PhRv, 109, 041101

Harris, H. C., Liebert, J., Kleinman, S. J., et al. 2003, AJ, 126, 1023

Healey, S. E., Romani, R. W., Cotter, G., et al. 2008, ApJS, 175, 97

Healey, S. E., Romani, R. W., Taylor, G. B., et al. 2007, ApJS, 171, 61

Hewett, P. C., Foltz, C. B., \& Chaffee, F. H. 1995, AJ, 109, 1498

Hincks, A. D., Acquaviva, V., Ade, P. A. R., et al. 2010, ApJS, 191, 423

Høg, E., Fabricius, C., Makarov, V. V., et al. 2000, A\&A, 355, L27

Holland, S. E., Bebek, C. J., Dawson, K. S., et al. 2006, Proc. SPIE, 6276, 10

Hopkins, P. F., Hernquist, L., Cox, T. J., et al. 2006, ApJS, 163, 1

Hopkins, P. F., Strauss, M. A., Hall, P. B., et al. 2004, AJ, 128, 1112

Horne, K. 1986, PASP, 98, 609

Huchra, J., Davis, M., Latham, D., \& Tonry, J. 1983, ApJS, 52, 89

Ivezić, Ž., Lupton, R. H., Schlegel, D., et al. 2004, AN, 325, 583

Ivezić, Ž., Smith, J. A., Miknaitis, G., et al. 2007, AJ, 134, 973

Jiang, L., Fan, X., Cool, R. J., et al. 2006, AJ, 131, 2788

Jones, D. H., Read, M. A., Saunders, W., et al. 2009, MNRAS, 399, 683

Kaiser, N. 1987, MNRAS, 227, 1

Kazin, E. A., Blanton, M. R., Scoccimarro, R., et al. 2010, ApJ, 710, 1444

Kelly, P. L., Hicken, M., Burke, D. L., Mandel, K. S., \& Kirshner, R. P. 2010, ApJ, 715,743

Kessler, R., Becker, A. C., Cinabro, D., et al. 2009, ApJS, 185, 32

Kim, T.-S., Viel, M., Haehnelt, M. G., Carswell, R. F., \& Cristiani, S. 2004, MNRAS, 347, 355

Kirkpatrick, J. A., Schlegel, D. J., Ross, N. P., et al. 2011, ApJ, 743, 125

Kleinman, S. J., Harris, H. C., Eisenstein, D. J., et al. 2004, ApJ, 607, 426

Komatsu, E., Smith, K. M., Dunkley, J., et al. 2011, ApJS, 192, 18

Kowalski, A. F., Hawley, S. L., Hilton, E. J., et al. 2009, AJ, 138, 633

Krughoff, K. S., Connolly, A. J., Frieman, J., et al. 2011, ApJ, 731, 42

Lampeitl, H., Nichol, R. C., Seo, H.-J., et al. 2010, MNRAS, 401, 2331 
Lawrence, A., Warren, S. J., Almaini, O., et al. 2007, MNRAS, 379, 1599 Le Goff, J. M., Magneville, C., Rollinde, E., et al. 2011, A\&A, 534, A135

Lee, K.-G., Bailey, S., Bartsch, L. E., et al. 2012, AJ, submitted (arXiv:1211.5146)

Lee, K.-G., Suzuki, N., \& Spergel, D. N. 2012, AJ, 143, 51

Lundgren, B. F., Brunner, R. J., York, D. G., et al. 2009, ApJ, 698, 819

Lundgren, B. F., Wilhite, B. C., Brunner, R. J., et al. 2007, ApJ, 656, 73

Lupton, R., Gunn, J. E., Ivezić, Z., et al. 2001, in ASP Conf. Ser. 238, Astronomical Data Analysis Software and Systems X, ed. F. R. Harnden, Jr., F. A. Primini, \& H. E. Payne (San Francisco, CA: ASP), 269

Manera, M., Scoccimarro, R., Percival, W. J., et al. 2012, MNRAS, in press (arXiv:1203.6609)

Maraston, C., Pforr, J., Henriques, B. M., et al. 2012, arXiv:1207.6114

Maraston, C., Strömbäck, G., Thomas, D., Wake, D. A., \& Nichol, R. C. 2009, MNRAS, 394, L107

Martin, D. C., Fanson, J., Schiminovich, D., et al. 2005, ApJ, 619, L1

Masters, K. L., Maraston, C., Nichol, R. C., et al. 2011, MNRAS, 418, 1055

McDonald, P. 2003, ApJ, 585, 34

McDonald, P., \& Eisenstein, D. J. 2007, PhRvD, 76, 063009

McDonald, P., Miralda-Escudé, J., Rauch, M., et al. 2000, ApJ, 543, 1

McDonald, P., Seljak, U., Burles, S., et al. 2006, ApJS, 163, 80

McDonald, P., Seljak, U., Cen, R., et al. 2005, ApJ, 635, 761

McGreer, I. D., Jiang, L., Fan, X., et al. 2012, BAAS, 219, 209.07

McQuinn, M., \& White, M. 2011, MNRAS, 415, 2257

Mehta, K. T., Cuesta, A. J., Xu, X., Eisenstein, D. J., \& Padmanabhan, N. 2012, arXiv: 1202.0092

Ménard, B., Nestor, D., Turnshek, D., et al. 2008, MNRAS, 385, 1053

Monet, D. G., Levine, S. E., Canzian, B., et al. 2003, AJ, 125, 984

Munn, J. A., Monet, D. G., Levine, S. E., et al. 2004, AJ, 127, 3034

Munn, J. A., Monet, D. G., Levine, S. E., et al. 2008, AJ, 136, 895

Murphy, D. N. A., Geach, J. E., \& Bower, R. G. 2012, MNRAS, 420, 1861

Noterdaeme, P., Petitjean, P., Carithers, W. C., et al. 2012, A\&A, accepted (arXiv:1210.1213)

Noterdaeme, P., Petitjean, P., Ledoux, C., \& Srianand, R. 2009, A\&A, 505, 1087

Padmanabhan, N., Schlegel, D. J., Finkbeiner, D. P., et al. 2008, ApJ, 674,1217

Padmanabhan, N., Xu, X., Eisenstein, D. J., et al. 2012, arXiv:1202.0090

Palanque-Delabrouille, N., Yeche, C., Myers, A. D., et al. 2011, A\&A, 530, A122

Parejko, J., Sunayama, T., Padmanabhan, N., et al. 2012, MNRAS, submitted (arXiv:1211.3976)

Pâris, I., Petitjean, P., Aubourg, E., et al. 2012, arXiv:1210.5166

Pâris, I., Petitjean, P., Rollinde, E., et al. 2011, A\&A, 530, A50

Peebles, P. J. E., \& Yu, J. T. 1970, ApJ, 162, 815

Percival, W. J., Reid, B. A., Eisenstein, D. J., et al. 2010, MNRAS, 401, 2148

Petrosian, V. 1976, ApJ, 209, L1

Pier, J. R., Munn, J. A., Hindsley, R. B., et al. 2003, AJ, 125, 1559

Reid, B. A., Samushia, L., White, M., et al. 2012, MNRAS, 426, 2719

Richards, G. T., Fan, X., Newberg, H. J., et al. 2002, AJ, 123, 2945

Richards, G. T., Myers, A. D., Gray, A. G., et al. 2009, ApJS, 180, 67

Richards, G. T., Strauss, M. A., Fan, X., et al. 2006, AJ, 131, 2766

Riess, A. G., Macri, L., Casertano, S., et al. 2011, ApJ, 730, 119

Roelofs, G. H. A., Nelemans, G., \& Groot, P. J. 2007, MNRAS, 382, 685

Ross, A. J., Percival, W. J., Sánchez, A. G., et al. 2012a, MNRAS, 424, 564

Ross, N. P., Myers, A. D., Sheldon, E. S., et al. 2012b, ApJS, 199, 3

Rots, A., Budavari, T., Szalay, A., \& Hagler, J. 2009, in Chandra's First Decade of Discovery, ed. S. Wolk, A. Fruscione, \& D. Swartz, abstract \#188 Sakharov, A. D. 1966, JETP, 22, 241

Sako, M., Bassett, B., Becker, A., et al. 2008, AJ, 135, 348

Sako, M., Bassett, B., Connolly, B., et al. 2011, ApJ, 738, 162

Sánchez, A. G., Scóccola, C. G., Ross, A. J., et al. 2012, MNRAS, 425, 415
Schlegel, D., Abdalla, F., Abraham, T., et al. 2011, arXiv:1106.1706

Schlegel, D., White, M., \& Eisenstein, D. 2009, Astro2010: The Astronomy and Astrophysics Decadal Survey, 314 (arXiv:0902.4680)

Schlegel, D. J., Finkbeiner, D. P., \& Davis, M. 1998, ApJ, 500, 525

Schmidt, K. B., Marshall, P. J., Rix, H.-W., et al. 2010a, ApJ, 714, 1194

Schmidt, S. J., West, A. A., Burgasser, A. J., Bochanski, J. J., \& Hawley, S. L. 2010b, AJ, 139, 1045

Schmidt, S. J., West, A. A., Hawley, S. L., \& Pineda, J. S. 2010c, AJ, 139, 1808

Schneider, D. P., Hall, P. B., Richards, G. T., et al. 2007, AJ, 134, 102

Schneider, D. P., Richards, G. T., Hall, P. B., et al. 2010, AJ, 139, 2360

Scholz, R.-D., Storm, J., Knapp, G. R., \& Zinnecker, H. 2009, A\&A, 494, 949

Seljak, U., Slosar, A., \& McDonald, P. 2006, JCAP, JCAP10(2006)014

Seo, H.-J., \& Eisenstein, D. J. 2003, ApJ, 598, 720

Seo, H.-J., \& Eisenstein, D. J. 2007, ApJ, 665, 14

Shectman, S. A., Landy, S. D., Oemler, A., et al. 1996, ApJ, 470, 172

Shu, Y., Bolton, A. S., Schlegel, D. J., et al. 2012, AJ, 143, 90

Skrutskie, M. F., Cutri, R. M., Stiening, R., et al. 2006, AJ, 131, 1163

Slosar, A., Font-Ribera, A., Pieri, M. M., et al. 2011, JCAP, JCAP09(2011)001

Smee, S., Gunn, J. E., Uomoto, A., et al. 2012, arXiv:1208.2233

Smith, J. A., Tucker, D. L., Kent, S., et al. 2002, AJ, 123, 2121

Stoughton, C., Lupton, R. H., Bernardi, M., et al. 2002, AJ, 123, 485

Strauss, M. A., Weinberg, D. H., Lupton, R. H., et al. 2002, AJ, 124, 1810

Sullivan, M., Conley, A., Howell, D. A., et al. 2010, MNRAS, 406, 782

Sunyaev, R. A., \& Zel'dovich, Y. B. 1970, Ap\&SS, 7, 3

Sunyaev, R. A., \& Zel'dovich, Y. B. 1972, CoASP, 4, 173

Swanson, M. E. C., Tegmark, M., Hamilton, A. J. S., \& Hill, J. C. 2008, MNRAS, 387,1391

Tal, T., Wake, D. A., van Dokkum, P. G., et al. 2012, ApJ, 746, 138

Thomas, D., et al. 2012, arXiv:1207.6115

Tojeiro, R., Percival, W. J., Brinkmann, J., et al. 2012, MNRAS, 424, 2339

Tremonti, C. A., Heckman, T. M., Kauffmann, G., et al. 2004, ApJ, 613, 898

Tucker, D. L., Kent, S., Richmond, M. W., et al. 2006, AN, 327, 821

Vanden Berk, D. E., Richards, G. T., Bauer, A., et al. 2001, AJ, 122, 549

Voges, W., Aschenbach, B., Boller, T., et al. 1999, A\&A, 349, 389

Voges, W., Aschenbach, B., Boller, T., et al. 2000, IAU Circ., 7432, 1

Watson, M. G., Schröder, A. C., Fyfe, D., et al. 2009, A\&A, 493, 339

Weinberg, D. H., Mortonson, M. J., Eisenstein, D. J., et al. 2012 , arXiv:1201.2434

West, A. A., Hawley, S. L., Bochanski, J. J., et al. 2008, AJ, 135, 785

West, A. A., Morgan, D. P., Bochanski, J. J., et al. 2011, AJ, 141, 97

West, A. A., Walkowicz, L. M., \& Hawley, S. L. 2005, PASP, 117, 706

Weymann, R. J., Morris, S. L., Foltz, C. B., \& Hewett, P. C. 1991, ApJ, 373, 23 White, M. 2003, arXiv:astro-ph/0305474

White, M., Blanton, M., Bolton, A., et al. 2011, ApJ, 728, 126

White, M., Myers, A. D., Ross, N. P., et al. 2012, MNRAS, 424, 933

White, M., Pope, A., Carlson, J., et al. 2010, ApJ, 713, 383

White, R. L., Becker, R. H., Gregg, M. D., et al. 2000, ApJS, 126, 133

Wild, V., Hewett, P. C., \& Pettini, M. 2007, MNRAS, 374, 292

Xu, X., Padmanabhan, N., Eisenstein, D. J., Mehta, K. T., \& Cuesta, A. J. 2012, arXiv: 1202.0091

Xue, X.-X., Rix, H.-W., Yanny, B., et al. 2011, ApJ, 738, 79

Yan, R. 2011, AJ, 142, 153

Yanny, B., Rockosi, C., Newberg, H. J., et al. 2009, AJ, 137, 4377

Yèche, C., Petitjean, P., Rich, J., et al. 2010, A\&A, 523, A14

York, D. G., Adelman, J., Anderson, J. E., Jr., et al. 2000, AJ, 120, 1579

York, D. G., Khare, P., Vanden, B. D., et al. 2006, MNRAS, 367, 945

York, D. G., vanden Berk, D., Richards, G. T., et al. 2005, in IAU Colloq. 199: Probing Galaxies through Quasar Absorption Lines, ed. P. Williams, C.-G Shu, \& B. Menard (Cambridge: Cambridge Univ. Press), 58

Zel'dovich, Y. B. 1970, A\&A, 5, 84 